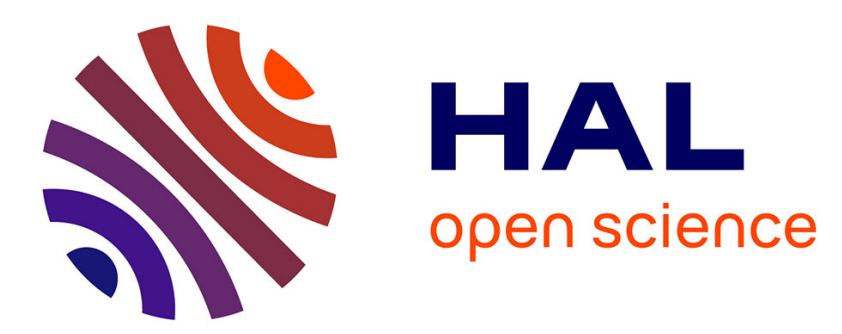

\title{
A multi-rate iterative coupling scheme for simulating dynamic ruptures and seismic waves generation in the prestressed earth
}

Ruichao Ye, Kundan Kumar, Maarten V. de Hoop, Michel Campillo

\section{- To cite this version:}

Ruichao Ye, Kundan Kumar, Maarten V. de Hoop, Michel Campillo. A multi-rate iterative coupling scheme for simulating dynamic ruptures and seismic waves generation in the prestressed earth. Journal of Computational Physics, 2020, 405, pp.109098. 10.1016/j.jcp.2019.109098 . hal-02928256

\section{HAL Id: hal-02928256 \\ https://hal.univ-grenoble-alpes.fr/hal-02928256}

Submitted on 7 Sep 2020

HAL is a multi-disciplinary open access archive for the deposit and dissemination of scientific research documents, whether they are published or not. The documents may come from teaching and research institutions in France or abroad, or from public or private research centers.
L'archive ouverte pluridisciplinaire HAL, est destinée au dépôt et à la diffusion de documents scientifiques de niveau recherche, publiés ou non, émanant des établissements d'enseignement et de recherche français ou étrangers, des laboratoires publics ou privés. 


\title{
A MULTI-RATE ITERATIVE COUPLING SCHEME FOR SIMULATING DYNAMIC RUPTURES AND SEISMIC WAVES GENERATION IN THE PRESTRESSED EARTH
}

\author{
RUICHAO YE *, KUNDAN KUMAR ${ }^{\dagger}$, MAARTEN V. DE HOOP $\ddagger$, AND MICHEL CAMPILLO $\S$
}

\begin{abstract}
We present a novel method to simulate the dynamic evolution of spontaneous ruptures governed by rate- and state-dependent friction laws and the interaction with seismic waves in a prestressed elastically deforming body. We propose a multi-rate iterative coupling scheme based on the variational form of the elastic-gravitational equations, and discretize employing a discontinuous Galerkin method, with nonlinear interior boundary conditions being weakly imposed across the fault surface as numerical fluxes. We introduce necessary interface jump penalty terms as well as an artificial viscous regularization, with the conditions for penalty and viscosity coefficients given based on an energy estimate and a convergence analysis. In the multi-rate scheme, an implicit-explicit Euler scheme in time is invoked, and the time step for the evolution of friction is chosen significantly finer than that for wave propagation and scattering. This is facilitated by the iterative scheme through the underlying decoupling where the linear, elastic wave equation plays the role of a Schur-complement to the friction model. A nonlinearly constrained optimization problem localized to each element on the rupture surface is then formulated and solved using the GaussNewton method. We test our algorithm on several benchmark examples and illustrate the generality of our method for realistic rupture simulations.
\end{abstract}

1. Introduction. The interaction of ruptures with seismic waves is of great interest in many areas of geophysical research and energy production pertaining to the study of natural earthquake source mechanisms, hydraulic fracturing and induced seismicity (e.g. $[41,57,15,35])$. As a matter of fact, the nucleation and propagation of ruptures vary distinctively both with friction laws (e.g. [12, $11,50,5,34,58])$ and rupture geometries (e.g. $[46,30,38,59])$. Numerical simulations of the rupture processes governed by elastic wave equation coupled with dynamic friction laws are challenging due to the low-regularity of solution space $[39,29,66]$. The nonlinear feedback of slip-rate into the friction coefficient $[48,63]$ makes the coupled system even more ill-conditioned. Nonetheless, various types of numerical methods have been used for such simulations, including the boundary integral (BI) method (e.g. $[25,37])$ which is based on layer potentials derived from fundamental solutions of elastic waves and restricts the rupture model to planar geometry and homogeneous material parameters on each side of the fault. Other numerical approaches have been designed for more general and realistic problems, allowing flexibility in the geometry of rupture surfaces and heterogeneity in material properties. We mention the finite difference (FD) method, with carefully designed curvilinear grids capturing the surface topography and rupture geometry (e.g. $[56,69,22,64])$, that has been widely used. An external weak representation of boundary conditions properly describing the coupling with friction laws is required. Commonly used methods in this category are summation by parts (SBP) difference operators (e.g.[33]), and hybridizing with numerical schemes with inherent boundary integrations (e.g. [42]).

However, the finite element (FE) method accommodates fully unstructured meshes with local refinements, allowing much more flexibility in characterizing the complex geometry of rupture surfaces. It relies on a weak formulation for the elastic wave equation as well as boundary conditions where coupling with dynamic friction is imposed (e.g. [36, 28, 1]. Traditional FE methods use linear basis functions and shared nodal points, which result in non-diagonal mass matrices and require techniques like mass-lumping for efficient solutions; these, however, may lead to nonphysical oscillation phenomena. The spectral element (SE) method addresses this problem by using tensor products of orthogonal polynomial basis functions. While sacrificing some of the freedom by choosing only hexahedral meshes, the SE method results in a diagonal mass matrix that can be trivially

* Department of Earth, Environmental and Planetary Sciences, Rice University, 6100 Main Street, Houston, TX 77005, USA

$\dagger$ Department of Mathematics, University of Bergen, Allegaten 41 Postboks 7803, 5020 Bergen, Norway

$\ddagger$ Department of Computational and Applied Mathematics, Rice University, 6100 Main Street, Houston, TX 77005, USA

$\S$ Institut des Sciences de la Terre, Université Grenoble Alpes, CNRS, IRD, SaintMartin-d'Héres, France 
inverted, and provides high polynomial order accuracy in wavefield simulations (e.g. [24]). Both FE and SE methods require node splitting locally on the rupture surface that allow for displacement discontinuity (e.g. $[31,63])$.

It seems more natural to solve problems with discontinuities, such as rupture dynamic problems, by using methods that completely split the domain into elements. Such methods are well known as the finite volume (FV) method (e.g. [8]) and the discontinuous Galerkin (DG) method (e.g. [20,61]), in which the nodes across the interface of two adjacent elements are distinct, and both the continuous and jumping boundary conditions are weakly imposed via numerical fluxes. There are multiple choices for the numerical flux, including the central flux (e.g. [55]), which is energy conservative but has drawback of generating spurious oscillations. An upwind flux is obtained as the solution to the Riemann problem on the interface, which takes the friction law and multi-physics coupling into account in a concise and self-consistent manner (e.g. $[19,43,67,62,68]$ ). Among other types of numerical fluxes are penalty-based schemes $(e . g .[49,17])$, which avoid the difficulty of diagonalizing the system with anisotropic materials that come with heterogeneity.

Our method of solving the coupled system of elastic wave equation and rate- and state-dependent friction law on a fault is based on our previous work on the DG method with modified penalty flux [65]. The novelty lies in three aspects. First, we avoid the usage of impedance, or the reliance on the Riemann solutions of any kind. Instead, we directly impose the distinct parts of the nonlinear friction law, the slip rate and the friction force, into the variational form as a slip boundary condition in a weak sense. The stability of this method is guaranteed by penalty terms as well as a viscosity coefficient, which is proportional to the time step that can be chosen small. Meanwhile, we consider the full Euler-Lagrange equations, which take into account the impact of the prestress and the initial gravitational potential on the field of motion. We invoke the so-called Cowling approximation in which the perturbation of gravitational potential induced by particle motion is ignored. However, the extension to include this perturbation, that is, transient gravity perturbations, is straightforwardly obtained by augmenting our system of equations with Poisson's equation (see e.g. [66]).

We prove convergence of our scheme based on a mixed strain-velocity formulation, for both continuous and discretized variational forms, in A and B, respectively. To deal with the nonlinear ordinary differential equation (ODE) for the friction state, which evolves much faster than particle motions in wave propagation and scattering, we utilize a multi-rate iterative coupling scheme. Such a scheme was developed for solving problems with different but coupled physical processes using different time steps. A relevant method is local time stepping (LTS), which is sometimes also called a multi-rate scheme (e.g. multi-rate Runge-kutta [21, 26]), and is usually focusing on obtaining an accurate numerical solution to a partial differential equation (PDE) using adaptive time steps based on local spatial refinements. One example of implementing a multi-rate scheme is Biot's model (e.g. [3]) coupling flow and geomechanics, where multiple finer time steps are taken for the stiff part of the flow within one coarse time step in the equation for elastic deformation. In a similar manner, in our system, we use coarse time steps for the elastic wave equation defined on a three-dimensional domain. Each coarse step is evenly divided into finer sub-steps, with which the state ODE defined on the fault surface is solved. The coupling of these two physical processes forms a nonlinear implicit system: the linear elastic-wave part takes the role of a Schur complement to the friction model that contains the state ODE and the friction law. By using the iterative coupling scheme, we rewrite solving the system as a nonlinearly constrained optimizaton problem defined elementwise on the rupture surface, and apply the Gauss-Newton method, where the gradient and Hessian matrix are formed and factorized locally (see $\mathrm{C}$ ). The overall algorithm greatly reduces the computation time of the solution to the large implicit nonlinear problem and, in fact, yields linear complexity.

While the focus of our paper is on spontaneous ruptures driven by prestress, we mention the relevance of our work to fracture problems, as these also involve slip boundary conditions. Like the rate- and state-dependent friction laws, fracture models include a feedback from slip to boundary tractions, but further allow normal jumps on the particle velocity across the fracturing boundary. A well-adopted law describing the fracture model is the linear slip (LS) boundary condition (e.g. [51, 
47])

$$
\left[\begin{array}{lll}
\kappa_{1} & & \\
& \kappa_{2} & \\
& & \kappa_{3}
\end{array}\right] \llbracket \boldsymbol{v} \rrbracket=\dot{\boldsymbol{\tau}}, \quad \kappa_{1}, \kappa_{2}, \kappa_{3}>0,
$$

where $\llbracket \boldsymbol{v} \rrbracket$ and $\boldsymbol{\tau}$ are the velocity jump and boundary tractions, respectively (see Section 2 for definitions), and " *" denotes time-derivative. As we can see in the definition of nonlinear functions $\mathcal{F}$ and $\mathcal{G}$ in (2.10) and (2.11) in Section 2, by taking $\kappa_{1} \rightarrow \infty$ and $\kappa_{2}=\kappa_{3}=\kappa>0$, the model turns into a simplified version of a rate- and state-dependent friction model, with $\mathcal{F}(\sigma, s, \psi)=\psi$ and $\mathcal{G}(s, \psi)=\kappa s$, which is also known as a linear slip-strengthening friction model. In the case where $\kappa$ is significantly negative, slip-weakening comes into place, simple explicit algorithms can hardly give converging solutions, and nonlinear iterations are usually required (e.g. [45, 22]).

2. The nonlinear boundary value problem in a weak form. We consider a 3-dimensional bounded domain $\Omega \subset \mathbb{R}^{3}$, which is representative of the earth with fully (anisotropic) elastic material. (We ignore anelastic effects.) We further assume that $\Omega$ is a disjoint union of Lipschitz subdomains $\Omega=\bigcup_{k=1}^{k_{0}} \Omega_{k}$, with interior boundaries given by

$$
\Sigma=\bigcup_{1 \leq k<k^{\prime} \leq I} \partial \Omega_{k} \cap \partial \Omega_{k^{\prime}} \backslash \partial \Omega
$$

We denote by $\Sigma_{\mathrm{c}}$ the non-slip solid-solid interfaces, and by $\Sigma_{\mathrm{f}}$ the slipping rupture surface. We choose $\boldsymbol{n}$ on $\Sigma \cup \partial \Omega$ as the unit normal vector of interior and exterior boundaries, which satisfies $\boldsymbol{n} \in L^{\infty}(\Sigma \cup \partial \Omega)^{3}$, and label the two sides across $\Sigma$ by "_" and " +". We denote the difference and average of any scalar or vector quantity $v$ across $\Sigma$ respectively by

$$
\llbracket v \rrbracket:=v^{+}-v^{-} \text {and }\{\{v\}\}:=\frac{1}{2}\left(v^{+}+v^{-}\right) .
$$

We use the subscript notation "(.)॥" for the tangential component with regards to $\boldsymbol{n}$, such that

$$
\boldsymbol{v}_{\|}:=\left(\boldsymbol{I}-\boldsymbol{n}^{\mathrm{T}} \boldsymbol{n}\right) \cdot \boldsymbol{v}=\boldsymbol{v}-(\boldsymbol{n} \cdot \boldsymbol{v}) \boldsymbol{n},
$$

where $\boldsymbol{I}$ is the $3 \times 3$ identity matrix.

2.1. The prestressed state. In the system of equations, we account for the reference gravitational potential, $\phi^{0}$, solving

$$
\Delta \Phi^{0}=4 \pi G \rho^{0},
$$

where $\rho^{0}$ is the initial density prior to the nucleation, and $G$ stands for the gravitational constant. $\rho^{0}$ is assumed uniformly bounded in $\Omega$ and away from zero, that is,

$$
0<C_{\rho^{0}} \leq\left\|\rho^{0}\right\|_{L^{\infty}(\Omega)} \leq C_{\rho^{0}}^{\star}
$$

By omitting rotation of the earth, we obtain the prestress tensor $\boldsymbol{T}^{0}$ that satisfies the mechanical equilibrium equation,

$$
\nabla \cdot \boldsymbol{T}^{0}=\rho^{0} \nabla \Phi^{0}=\rho^{0} \boldsymbol{g}^{0}
$$

where $\boldsymbol{g}^{0}:=\nabla \Phi^{0}$ is the gravitational acceleration. We expect that $\Phi^{0} \in W^{2, \infty}$ everywhere in $\mathbb{R}^{3}$ (by regularizing $\rho^{0}$ if necessary), which means that the gradient of gravitational acceleration as well as the divergence of prestress are bounded everywhere, that is,

$$
\left\|\nabla \boldsymbol{g}^{0}\right\|_{L^{\infty}\left(\mathbb{R}^{3}\right)}=\left\|\nabla \nabla \Phi^{0}\right\|_{L^{\infty}\left(\mathbb{R}^{3}\right)} \leq C_{\Phi^{0}}, \quad \text { and } \quad\left\|\nabla \cdot \boldsymbol{T}^{0}\right\|_{L^{\infty}\left(\mathbb{R}^{3}\right)} \leq\left\|\rho^{0}\right\|_{L^{\infty}\left(\mathbb{R}^{3}\right)}\left\|\nabla \Phi^{0}\right\|_{L^{\infty}\left(\mathbb{R}^{3}\right)} \leq C_{\rho^{0}}^{\star} C_{\Phi^{0}} .
$$




\begin{tabular}{|l|l|l|}
\hline symbols & physical meaning & reference equation \\
\hline$\rho^{0}$ & initial density & $(2.1)$ \\
\hline$\Phi^{0}$ & initial gravitational potential & $(2.1)$ \\
\hline $\boldsymbol{T}^{0}$ & prestress tensor & $(2.2)$ \\
\hline $\boldsymbol{g}^{0}$ & gravitational acceleration & $(2.2)$ \\
\hline $\boldsymbol{u}$ & particle displacement & $(2.4)$ \\
\hline $\boldsymbol{v}$ & particle velocity & $(2.4)$ \\
\hline $\boldsymbol{E}$ & strain tensor & $(2.4)$ \\
\hline $\boldsymbol{C}$ & isentropic stiffness tensor & $(2.5)$ \\
\hline $\boldsymbol{\Lambda}^{\boldsymbol{T}^{0}}$ & elastic tensor & $(2.5)$ \\
\hline $\boldsymbol{T}^{\text {PK1 }}$ & first Piola-Kirchhoff stress tensor & $(2.6)$ \\
\hline $\boldsymbol{s}$ & slip velocity on rupture surface & $(2.7)$ \\
\hline $\boldsymbol{s}$ & slip-rate on rupture surface & $(2.7)$ \\
\hline $\boldsymbol{n}^{s}$ & instantaneous (Eulerian) normal direction & $(2.8)$ \\
\hline $\boldsymbol{T}^{s}$ & (Eulerian) Cauchy stress & $(2.8)$ \\
\hline $\boldsymbol{\tau}^{s}$ & instantaneous (Eulerian) traction on rupture surface & $(2.8)$ \\
\hline $\boldsymbol{\tau}_{\mathrm{f}}$ & friction force on rupture surface & $(2.9)$ \\
\hline
\end{tabular}

Table 2.1: List of symbols.

We invoke the Cowling approximation, that is, ignore the perturbation of gravitational potential generated by mass redistribution. Before the nucleation of a rupture, the system is in a steady state with force equilibrium and zero particle displacement and velocity. The spontaneous rupture then occurs as the material fails at some locations of a pre-existing known fault plane $\left(\Sigma_{\mathrm{f}}\right)$, and the rupture spreads catastrophically to adjacent regions, which is also called the "propagation" of rupture (e.g. [14, p. 187]). The consideration of a time evolving $\Sigma_{\mathrm{f}}$ is a delicate issue that is outside the scope of this paper.

We denote by $\boldsymbol{u}$ the particle displacement, and by

$$
\boldsymbol{v}:=\dot{\boldsymbol{u}} \text { and } \boldsymbol{E}:=\nabla \boldsymbol{u}
$$

the particle velocity and strain tensor, respectively. We write $\boldsymbol{\Lambda}^{\boldsymbol{T}^{0}}$ for the elastic tensor which depends on $\boldsymbol{T}^{0}$ and the in situ isentropic stiffness tensor $\boldsymbol{C}$,

$$
\Lambda_{i j k l}^{\boldsymbol{T}^{0}}=C_{i j k l}+\frac{1}{2}\left(T_{i j}^{0} \delta_{k l}+T_{k l}^{0} \delta_{i j}+T_{i k}^{0} \delta_{j l}-T_{j l}^{0} \delta_{i k}-T_{j k}^{0} \delta_{i l}-T_{i l}^{0} \delta_{j k}\right)
$$

such that $\boldsymbol{T}^{\mathrm{PK} 1}=\boldsymbol{\Lambda}^{\boldsymbol{T}^{0}}: \boldsymbol{E}$ stands for the first Piola-Kirchhoff stress perturbation. In other words,

$$
\boldsymbol{T}^{\mathrm{PK} 1}=\boldsymbol{C}: \boldsymbol{E}+\frac{1}{2}\left(\boldsymbol{T}^{0} \operatorname{Tr}(\boldsymbol{E})+\boldsymbol{I}\left(\boldsymbol{T}^{0}: \boldsymbol{E}\right)+\boldsymbol{T}^{0} \boldsymbol{E}-\left(\boldsymbol{T}^{0} \boldsymbol{E}\right)^{\mathrm{T}}-\left(\boldsymbol{E} \boldsymbol{T}^{0}\right)^{\mathrm{T}}-\boldsymbol{E} \boldsymbol{T}^{0}\right) .
$$

2.2. Dynamic boundary conditions. We define the slip velocity and its magnitude, the slip-rate, by

$$
s:=\llbracket \boldsymbol{v}_{\|} \rrbracket=\boldsymbol{v}_{\|}^{+}-\boldsymbol{v}_{\|}^{-}, \quad s:=|s| .
$$


The particle displacement and the Cauchy stress on $\Sigma_{\mathrm{f}}$ satisfy the non-opening slip boundary conditions $[14,(2.80)$ and $(2.81)]$,

$$
\llbracket \boldsymbol{n}^{s} \cdot \boldsymbol{u} \rrbracket=0, \quad \llbracket \boldsymbol{n}^{s} \cdot \boldsymbol{T}^{s} \rrbracket=0, \quad \text { on } \Sigma_{\mathrm{f}} .
$$

We denote by $\boldsymbol{\tau}^{s}=\boldsymbol{n}^{s} \cdot \boldsymbol{T}^{s}$ the total traction in the Eulerian representation. The force balancing on the rupture surface requires that the tangential component of $\boldsymbol{\tau}^{s}$ equates the friction force, that is, $\left(\boldsymbol{n}^{s} \cdot \boldsymbol{T}^{s}\right)_{\|}=\boldsymbol{\tau}_{\mathrm{f}}$, the direction of which is opposite to the slip velocity, that is (e.g. Day et al. (2005) [16, (4)], Moczo et al. (2014) [40, p. 60]),

$$
\tau_{\mathrm{f}} \boldsymbol{s}-s \boldsymbol{\tau}_{\mathrm{f}}=0,
$$

where $\tau_{\mathrm{f}}:=\left|\boldsymbol{\tau}_{\mathrm{f}}\right|$ signifies the magnitude of friction force. We focus on the Dieterich-Ruina friction law discussed in Rice et al. [48] depending on compressive stress, slip-rate and state variable,

$$
\tau_{\mathrm{f}}=\mathcal{F}(\sigma, s, \psi),
$$

in which $\psi$ describes the maturity of rupture, and satisfies the ordinary differential relation

$$
\dot{\psi}+\mathcal{G}(s, \psi)=0 .
$$

We make the following assumptions regarding the rate- and state-dependent friction law (see also $[66$, Section 2]),

$$
\begin{aligned}
& 0<C_{\mathcal{F}, \sigma} \leq \frac{\partial \mathcal{F}(\sigma, s, \psi)}{\partial \sigma} \leq C_{\mathcal{F}, \sigma}^{\star}, \\
& 0<C_{\mathcal{F}, \psi} \leq \frac{\partial \mathcal{F}(\sigma, s, \psi)}{\partial \psi} \leq C_{\mathcal{F}, \psi}^{\star}, \quad 0<C_{\mathcal{F}, s} \leq \frac{\partial \mathcal{F}(\sigma, s, \psi)}{\partial s} \leq C_{\mathcal{F}, s}^{\star}, \\
& 0 \leq C_{\mathcal{G}, \psi} \leq \frac{\partial \mathcal{G}(s, \psi)}{\partial \psi} \leq C_{\mathcal{G}, \psi}^{\star}, \quad \text { and } \quad\left|\frac{\partial \mathcal{G}(s, \psi)}{\partial s}\right| \leq C_{\mathcal{G}, s}^{\star}, \\
& \text { for all } \sigma \in \mathbb{R}^{+}, s, \psi \in[0, \infty) .
\end{aligned}
$$

The dynamic boundary condition for the first Piola-Kirchoff stress can be derived from (2.8) and yields to first-order approximation (e.g. [14, p. 68], [10, p. 47])

$$
\llbracket \boldsymbol{n} \cdot \boldsymbol{T}^{\mathrm{PK} 1}-\nabla^{\Sigma} \cdot\left(\boldsymbol{u}\left(\boldsymbol{n} \cdot \boldsymbol{T}^{0}\right)\right) \rrbracket=0 . \quad \llbracket \boldsymbol{n} \cdot \boldsymbol{u} \rrbracket=0 \quad \text { on } \Sigma_{\mathrm{f}},
$$

where $\nabla^{\Sigma}:=\nabla-\boldsymbol{n} \partial_{n}$ is the surface gradient. Following the same procedure, one can also derive that

$$
\boldsymbol{\tau}^{s} \approx \boldsymbol{n} \cdot \boldsymbol{T}^{\mathrm{PK} 1}+\boldsymbol{n} \cdot \boldsymbol{T}^{0}-\nabla^{\Sigma} \cdot\left(\boldsymbol{u}\left(\boldsymbol{n} \cdot \boldsymbol{T}^{0}\right)\right) .
$$

We also write the dynamic boundary conditions on $\Sigma_{\mathrm{c}}$, the solid-solid interfaces, with standard continuity conditions for the traction and the particle velocity (e.g. [14, (2.79) and (3.65)])

$$
\llbracket \boldsymbol{n} \cdot \boldsymbol{T}^{\mathrm{PK} 1} \rrbracket=0, \quad \llbracket \boldsymbol{u} \rrbracket=0, \quad \text { on } \Sigma_{\mathrm{c}},
$$

and based on the continuity of prestress $\llbracket \boldsymbol{n} \cdot \boldsymbol{T}^{0} \rrbracket=0$, one has

$$
\llbracket \boldsymbol{n} \cdot(\boldsymbol{C}: \boldsymbol{E}) \rrbracket=0, \quad \text { on } \Sigma_{\mathrm{c}} .
$$

We assume that the rupture remains compressive, or, in other words, the compressive normal stress $\sigma$ is positive throughout time. Therefore, $\sigma=-\boldsymbol{n} \cdot \boldsymbol{T}^{s} \cdot \boldsymbol{n}$ if the trace of $\boldsymbol{T}^{s}$ is positive in tension. 
2.3. Energy spaces. We denote by $L^{2}(\Omega)$ the space of square integrable functions on the Lipschitz composite domain $\Omega$ defined in Section 2. We define the Sobolev space

$$
\mathbb{H}:=\left\{\boldsymbol{v} \in L^{2}(\Omega) \mid \sum_{k=1}^{k_{0}}\|\nabla \boldsymbol{v}\|_{L^{2}\left(\Omega_{k}\right)}^{2}<\infty\right\},
$$

with the norm

$$
\|\boldsymbol{v}\|_{\mathbb{H}}:=\left(\sum_{k=1}^{k_{0}}\|\boldsymbol{v}\|_{H^{1}\left(\Omega_{k}\right)}^{2}\right)^{\frac{1}{2}}
$$

With the assumption that the material parameters belong to $L^{\infty}(\Omega)$, and that the deviatoric part of $\boldsymbol{T}^{0}$ is small enough such that $\boldsymbol{\Lambda}^{\boldsymbol{T}^{0}}$ is coercive (see [18, 66]), we define the following weighted inner products,

$$
\begin{aligned}
(\boldsymbol{v}, \boldsymbol{w})_{L^{2}\left(\Omega ; \rho^{0}\right)} & :=\sum_{k=1}^{k_{0}} \int_{\Omega_{k}}(\boldsymbol{v} \cdot \boldsymbol{w}) \rho^{0} \mathrm{~d} \Omega, \\
(\boldsymbol{E}, \boldsymbol{H})_{L^{2}\left(\Omega ; \boldsymbol{T}^{\left.T^{0}\right)}\right.} & :=\sum_{k=1}^{k_{0}} \int_{\Omega_{k}} \boldsymbol{H}:\left(\boldsymbol{\Lambda}^{\boldsymbol{T}^{0}}: \boldsymbol{E}\right) \mathrm{d} \Omega,
\end{aligned}
$$

with corresponding weighted norms that have the following equivalences,

$$
\begin{aligned}
& C_{\rho^{0}}\|\boldsymbol{u}\|_{L^{2}(\Omega)}^{2} \leq\|\boldsymbol{u}\|_{L^{2}\left(\Omega ; \rho^{0}\right)}^{2} \leq C_{\rho^{0}}^{\star}\|\boldsymbol{u}\|_{L^{2}(\Omega)}^{2}, \\
& C_{\boldsymbol{\Lambda}}\|\boldsymbol{E}\|_{L^{2}(\Omega)}^{2} \leq\|\boldsymbol{E}\|_{L^{2}\left(\Omega ; \boldsymbol{A}^{T^{0}}\right)}^{2} \leq C_{\boldsymbol{\Lambda}}^{\star}\|\boldsymbol{E}\|_{L^{2}(\Omega)}^{2} .
\end{aligned}
$$

We introduce the space for the weak solution as

$$
\begin{aligned}
& V_{1}=\left\{\boldsymbol{u} \in L^{\infty}([0, T] ; \mathbb{H}) \mid \begin{array}{l}
\dot{\boldsymbol{u}} \in L^{2}([0, T] ; \mathbb{H}) \cap L^{\infty}\left([0, T] ; L^{2}(\Omega)\right), \\
\ddot{\boldsymbol{u}} \in L^{2}\left([0, T] ; \mathbb{H}^{\prime}\right)
\end{array}\right\}, \\
& V_{2}=\left\{\boldsymbol{E} \in L^{\infty}\left([0, T] ; L^{2}(\Omega)\right) \mid \dot{\boldsymbol{E}} \in L^{2}\left([0, T] ; L^{2}(\Omega)\right)\right\}, \\
& V_{3}=\left\{\psi \in L^{\infty}\left([0, T] ; L^{2}\left(\Sigma_{\mathrm{f}}\right)\right) \mid \dot{\psi} \in L^{2}\left([0, T] ; L^{2}\left(\Sigma_{\mathrm{f}}\right)\right)\right\} .
\end{aligned}
$$

In the above, $\mathbb{H}^{\prime}$ is the dual space of $\mathbb{H}$. We let $P_{p}$ be the space of polynomials of degree lower than or equal to $p$. Based on the Weierstrass approximation theorem, $\bigcup_{p=1}^{\infty} P_{p}$ is dense in $L^{2}$. For the Lipschitz composite domain $\Omega=\bigcup_{k=1}^{I} \Omega_{k}$, we define space $\mathcal{P}_{p}$ such that

$$
\mathcal{P}_{p}(\Omega):=\left\{v \in L^{2}(\Omega)|v|_{\Omega_{k}} \in P_{p}\left(\Omega_{k}\right), \quad \text { for } k=1,2, \cdots, I\right\} .
$$

In other words, $\mathcal{P}_{p}(\Omega)$ is the space of piecewise smooth functions which are polynomials of degree at most $p$ within each Lipschitz subdomain of $\Omega$. We furthermore denote $V_{1}^{p}:=V_{1} \cap \mathcal{P}_{p}(\Omega)$, $V_{2}^{p}:=V_{2} \cap \mathcal{P}_{p}(\Omega)$ and $V_{3}^{p}:=V_{3} \cap \mathcal{P}_{p}(\Omega)$.

Following Arnold et al. [7], one can define a linear continuous map (a lifting operator) $\boldsymbol{r}_{\mathrm{f}}: V_{1} \rightarrow$ $\mathcal{P}_{p}(\Omega)$ such that

$$
\int_{\Omega} \boldsymbol{r}_{\mathrm{f}}(\boldsymbol{v}): \boldsymbol{H} \mathrm{d} \Omega=\int_{\Sigma_{\mathrm{f}}} \llbracket \boldsymbol{n} \cdot \boldsymbol{v} \rrbracket\{\{\boldsymbol{n} \cdot \boldsymbol{H} \cdot \boldsymbol{n}\}\} \mathrm{d} \Sigma, \quad \forall \boldsymbol{H} \in \mathcal{P}_{p}(\Omega) .
$$

We use the operator $\boldsymbol{r}_{\mathrm{f}}$ in composing the penalty-based weak form in the following section. 
2.4. Weak form of the coupled system. In a companion paper [66], we present and analyze the variational form of the system coupling the elastic-gravitational system of equations with rupture dynamics. Here, we introduce the mixed strain-velocity variational form by introducing the strain tensor $\boldsymbol{E}$, the symmetrized gradient of particle displacement, as an unknown that allows us to compute the stress in a more direct way. We recall the strong form of particle motion in the Cowling approximation as

$$
\rho^{0}\left(\ddot{\boldsymbol{u}}+\boldsymbol{u} \cdot\left(\nabla \boldsymbol{g}^{0}\right)\right)-\nabla \cdot \boldsymbol{T}^{\mathrm{PK} 1}=0 .
$$

The first order hyperbolic system yielding (2.21) as well as the equations on the interior boundaries in (2.9)-(2.14) are rewritten weakly as follows.

Problem 1. Find $(\boldsymbol{u}, \boldsymbol{E}, \psi) \in V_{1} \times V_{2} \times V_{3}$ such that

$$
\begin{aligned}
\int_{\Omega} \rho^{0}\left(\ddot{\boldsymbol{u}}+\boldsymbol{u} \cdot\left(\nabla \nabla \Phi^{0}\right)\right) \cdot \boldsymbol{w} \mathrm{d} \Omega+\int_{\Omega}\left(\boldsymbol{\Lambda}^{\boldsymbol{T}^{0}}: \boldsymbol{E}\right): \nabla \boldsymbol{w} \mathrm{d} \Omega \\
\quad+\frac{\gamma \int_{\Omega}((\dot{\boldsymbol{E}}: \nabla \boldsymbol{w})+(\dot{\boldsymbol{u}} \cdot \boldsymbol{w})) \mathrm{d} \Omega}{}+\alpha_{\mathrm{f}} \int_{\Omega} \boldsymbol{r}_{\mathrm{f}}(\boldsymbol{u}+\dot{\boldsymbol{u}}): \boldsymbol{r}_{\mathrm{f}}(\boldsymbol{w}) \mathrm{d} \Omega \\
\quad+\int_{\Sigma_{\mathrm{f}}} \boldsymbol{\tau}_{\mathrm{f}} \cdot \llbracket \boldsymbol{w}_{\|} \rrbracket \mathrm{d} \Sigma-\int_{\Sigma_{\mathrm{f}}} \sigma \llbracket \boldsymbol{n} \cdot \boldsymbol{w} \rrbracket \mathrm{d} \Sigma-\int_{\Sigma_{\mathrm{f}}} \llbracket \boldsymbol{\tau}_{2} \cdot \boldsymbol{w} \rrbracket \mathrm{d} \Sigma \\
=\int_{\Sigma_{\mathrm{f}}}\left(\boldsymbol{n} \cdot\left(\boldsymbol{T}^{0}+\boldsymbol{T}^{\delta}\right)\right) \cdot \llbracket \boldsymbol{w} \rrbracket \mathrm{d} \Sigma,
\end{aligned}
$$

$$
\int_{\Omega} \dot{\boldsymbol{E}}: \boldsymbol{H} \mathrm{d} \Omega+\int_{\Omega} \dot{\boldsymbol{u}} \cdot(\nabla \cdot \boldsymbol{H}) \mathrm{d} \Omega+\int_{\Sigma_{\mathrm{f}}}\{\{\dot{\boldsymbol{u}}\}\} \cdot \llbracket \boldsymbol{n} \cdot \boldsymbol{H} \rrbracket \mathrm{d} \Sigma+\int_{\Sigma_{\mathrm{f}}} \boldsymbol{s} \cdot\{\{\boldsymbol{n} \cdot \boldsymbol{H}\}\} \mathrm{d} \Sigma=0,
$$

$$
\int_{\Sigma_{\mathrm{f}}} \dot{\psi} \varphi \mathrm{d} \Sigma+\int_{\Sigma_{\mathrm{f}}} \mathcal{G}(s, \psi) \varphi \mathrm{d} \Sigma=0
$$

with

$$
\begin{aligned}
& \boldsymbol{s}=\llbracket \boldsymbol{u}_{\|} \rrbracket, \quad s:=|\boldsymbol{s}|, \\
& \boldsymbol{\tau}_{2}+\nabla^{\Sigma} \cdot\left(\boldsymbol{u}\left(\boldsymbol{n} \cdot \boldsymbol{T}^{0}\right)\right)=0, \\
& \sigma+\boldsymbol{n} \cdot\left(\boldsymbol{n} \cdot\left(\boldsymbol{T}^{0}+\boldsymbol{T}^{\delta}+\left\{\left\{\boldsymbol{\Lambda}^{\boldsymbol{T}^{0}}: \boldsymbol{E}\right\}\right\}\right)+\left\{\left\{\boldsymbol{\tau}_{2}\right\}\right\}\right)=0, \\
& \mathcal{F}(\sigma, s, \psi) \boldsymbol{s}-s \boldsymbol{\tau}_{\mathrm{f}}=0,
\end{aligned}
$$

holds for all $(\boldsymbol{w}, \boldsymbol{H}, \varphi) \in V_{1}^{p} \times V_{2}^{p} \times V_{3}^{p}$.

The boxed terms in (2.22a) indicate a viscosity regularization term and a boundary penalty term, with the viscosity coefficient denoted by $\gamma$ and the penalty coefficient by $\alpha_{\mathrm{f}}$, both being positive constants. The above formulation assumes that $\Omega$ is a sufficiently large open set such that the boundary integration term on $\partial \Omega$ vanishes. Nevertheless, including such a term (e.g. a traction-free boundary, see Section 5) brings no issue in both analysis and numerical implementations. We give the precise criteria for $\gamma$ and $\alpha_{\mathrm{f}}$ ensuring the well-posedness of this coupled weak formulation in $\mathrm{A}$. 
3. The discontinuous Galerkin method with implicit time discretization. We partition the domain $\Omega$ into tetrahedral finite elements, $\Omega=\bigcup \Omega^{\mathrm{e}}$, such that the unstructured tetrahedral mesh is consistent with the geometry, that is, $\Sigma \subset \bigcup \partial \Omega^{\mathrm{e}}$. We distinguish the facets attached to the rupture plane with slip boundary conditions by $\Sigma_{\mathrm{f}}^{\mathrm{e}}$, and thus $\Sigma_{\mathrm{f}}=\bigcup \Sigma_{\mathrm{f}}^{\mathrm{e}}$. All other facets of the interior elements are denoted by $\Sigma_{\mathrm{c}}^{\mathrm{e}}$. We set

$$
\begin{aligned}
V_{1 h}^{p} & =\left\{\boldsymbol{u} \in V_{1}\left|u_{i}\right|_{\Omega^{\mathrm{e}}} \in P_{p}\left(\Omega^{\mathrm{e}}\right), \quad i \in\{1,2,3\}\right\}, \\
V_{2 h}^{p} & =\left\{\boldsymbol{E} \in V_{2}\left|E_{i j}\right|_{\Omega^{\mathrm{e}}} \in P_{p}\left(\Omega^{\mathrm{e}}\right), \quad i, j \in\{1,2,3\}\right\}, \\
V_{3 h}^{p} & =\left\{\psi \in V_{3}|\psi|_{\Sigma_{\mathrm{f}}^{\mathrm{e}}} \in P_{p}\left(\Sigma_{\mathrm{f}}^{\mathrm{e}}\right)\right\} .
\end{aligned}
$$

To simplify the analysis, we assume that the elastic parameters are piecewise constant, that is,

$$
\rho_{h}^{0},\left(\boldsymbol{\Lambda}_{h}^{\boldsymbol{T}^{0}}\right)_{i j k l},\left(\boldsymbol{T}_{h}^{0}\right)_{i j} \in\left\{\varphi \in L^{\infty}(\Omega)|\varphi|_{\Omega^{\mathrm{e}}} \in P_{0}\left(\Omega^{\mathrm{e}}\right)\right\}, \quad i, j, k, l \in\{1,2,3\}
$$

and that

$$
\Phi_{h}^{0} \in\left\{\Phi \in W^{2, \infty}\left(\mathbb{R}^{3}\right)|\Phi|_{\Omega^{\mathrm{e}}} \in P_{2}\left(\Omega^{\mathrm{e}}\right)\right\}
$$

such that $\boldsymbol{K}_{h}:=\nabla_{h} \nabla_{h} \Phi_{h}^{0}$ is piecewise constant, where $\nabla_{h}$ is the gradient of polynomials within $\Omega^{\mathrm{e}}$. Instead of using universal constants $\alpha_{\mathrm{f}}$ and $\gamma$, we implement elementwise coefficients $\alpha_{\mathrm{f}}^{\mathrm{e}}$ and $\gamma^{\mathrm{e}}$, which satisfy the conditions stated by Theorem B.1 in B. A constant $\alpha_{\mathrm{c}}^{\mathrm{e}}>0$ is also introduced as a penalty coefficient on continuous boundary conditions that enforce the coercivity of the DG formulation (see details in Ye et al. (2016) [65]). The semi-discretized DG formulation is therefore obtained as follows

Problem 2. Find $\left(\boldsymbol{u}_{h}, \boldsymbol{E}_{h}, \psi_{h}\right) \in V_{1 h}^{p} \times V_{2 h}^{p} \times V_{3 h}^{p}$ such that

$$
\begin{aligned}
& \sum_{\Omega^{\mathrm{e}}} \int_{\Omega^{\mathrm{e}}}\left(\left(\rho_{h}^{0}\left(\ddot{\boldsymbol{u}}_{h}+\boldsymbol{u}_{h} \cdot \boldsymbol{K}_{h}\right)+\gamma^{\mathrm{e}} \dot{\boldsymbol{u}}_{h}\right) \cdot \boldsymbol{w}_{h}+\left(\left(\boldsymbol{\Lambda}_{h}^{\boldsymbol{T}^{0}}: \boldsymbol{E}_{h}+\gamma^{\mathrm{e}} \dot{\boldsymbol{E}}_{h}\right): \nabla \boldsymbol{w}_{h}\right)\right) \mathrm{d} \Omega \\
& \quad+\sum_{\Sigma_{\mathrm{f}}^{\mathrm{e}}} \int_{\Sigma_{\mathrm{f}}^{\mathrm{e}}}\left(\left(\boldsymbol{\tau}_{\mathrm{f} h}-\sigma_{h} \boldsymbol{n}\right) \cdot \llbracket \boldsymbol{w}_{h} \rrbracket-\llbracket \boldsymbol{\tau}_{2 h} \cdot \boldsymbol{w}_{h} \rrbracket+\alpha_{\mathrm{f}}^{\mathrm{e}} \llbracket \boldsymbol{n} \cdot\left(\boldsymbol{u}_{h}+\dot{\boldsymbol{u}}_{h}\right) \rrbracket \llbracket \boldsymbol{n} \cdot \boldsymbol{w}_{h} \rrbracket\right) \mathrm{d} \Sigma \\
& \quad+\sum_{\Sigma_{\mathrm{c}}^{\mathrm{e}}} \int_{\Sigma_{\mathrm{c}}^{\mathrm{e}}}\left(\left\{\left\{\boldsymbol{n} \cdot\left(\boldsymbol{\Lambda}_{h}^{\boldsymbol{T}^{0}}: \boldsymbol{E}_{h}\right)\right\}\right\}+\alpha_{\mathrm{c}}^{\mathrm{e}} \llbracket \dot{\boldsymbol{u}}_{h} \rrbracket\right) \cdot \llbracket \boldsymbol{w}_{h} \rrbracket \mathrm{d} \Sigma=\sum_{\Sigma_{\mathrm{f}}^{\mathrm{e}}} \int_{\Sigma_{\mathrm{f}}^{\mathrm{e}}}\left(\boldsymbol{n} \cdot\left(\boldsymbol{T}_{h}^{0}+\boldsymbol{T}_{h}^{\delta}\right)\right) \cdot \llbracket \boldsymbol{w}_{h} \rrbracket \mathrm{d} \Sigma,
\end{aligned}
$$

$$
\begin{aligned}
& \sum_{\Omega^{\mathrm{e}}} \int_{\Omega^{\mathrm{e}}}\left(\dot{\boldsymbol{E}}_{h}: \boldsymbol{H}_{h}+\dot{\boldsymbol{u}}_{h} \cdot\left(\nabla \cdot \boldsymbol{H}_{h}\right)\right) \mathrm{d} \Omega+\sum_{\Sigma_{\mathrm{f}}^{\mathrm{e}}} \int_{\Sigma_{\mathrm{f}}^{\mathrm{e}}}\left(\left\{\left\{\dot{\boldsymbol{u}}_{h}\right\}\right\} \cdot \llbracket \boldsymbol{n} \cdot \boldsymbol{H}_{h} \rrbracket+\boldsymbol{s}_{h} \cdot\left\{\left\{\boldsymbol{n} \cdot \boldsymbol{H}_{h}\right\}\right\}\right) \mathrm{d} \Sigma \\
& \quad+\sum_{\Sigma_{\mathrm{c}}^{\mathrm{e}}} \int_{\Sigma_{\mathrm{c}}^{\mathrm{e}}}\left(\left\{\left\{\dot{\boldsymbol{u}}_{h}\right\}\right\}+\alpha_{\mathrm{c}}^{\mathrm{e}} \llbracket \boldsymbol{n} \cdot\left(\boldsymbol{\Lambda}_{h}^{\boldsymbol{T}^{0}}: \boldsymbol{E}_{h}\right) \rrbracket\right) \cdot \llbracket \boldsymbol{n} \cdot \boldsymbol{H}_{h} \rrbracket \mathrm{d} \Sigma=0,
\end{aligned}
$$

$$
\int_{\Sigma_{\mathrm{f}}^{\mathrm{e}}} \dot{\psi}_{h} \varphi_{h} \mathrm{~d} \Sigma+\int_{\Sigma_{\mathrm{f}}^{\mathrm{e}}} \mathcal{G}\left(s_{h}, \psi_{h}\right) \varphi_{h} \mathrm{~d} \Sigma=0
$$




$$
\begin{aligned}
\boldsymbol{\tau}_{2 h}+\nabla^{\Sigma} \cdot\left(\boldsymbol{u}_{h}\left(\boldsymbol{n} \cdot \boldsymbol{T}_{h}^{0}\right)\right) & =0, \\
\sigma_{h}+\boldsymbol{n} \cdot\left(\boldsymbol{n} \cdot\left(\boldsymbol{T}_{h}^{0}+\boldsymbol{T}_{h}^{\delta}+\left\{\left\{\boldsymbol{\Lambda}_{h}^{\boldsymbol{T}^{0}}: \boldsymbol{E}_{h}\right\}\right\}\right)+\left\{\left\{\boldsymbol{\tau}_{2 h}\right\}\right\}\right) & =0, \\
\boldsymbol{s}_{h}=\llbracket \dot{\boldsymbol{u}}_{h \|} \rrbracket, \quad s_{h}:=\left|\boldsymbol{s}_{h}\right|, & \text { on } \Sigma_{\mathrm{f}}^{\mathrm{e}} . \\
\boldsymbol{s}_{h} \mathcal{F}\left(\sigma_{h}, s_{h}, \psi_{h}\right)-s_{h} \boldsymbol{\tau}_{\mathrm{f} h} & =0,
\end{aligned}
$$

for all test functions $\left(\boldsymbol{H}_{h}, \boldsymbol{w}_{h}, \varphi_{h}\right) \in V_{1 h}^{p} \times V_{2 h}^{p} \times V_{3 h}^{p}$.

We use the particle velocity $\boldsymbol{v}_{h}=\dot{\boldsymbol{u}}_{h}$, and discretize the time interval with a uniform time step $\Delta t=\frac{T}{N_{T}}$, and write $t_{n}=n \Delta t$. The time discretization is indicated by the superscript ${ }^{(n)}$. We introduce

$$
\begin{aligned}
& \hat{V}_{1 h}^{p}=\left\{\boldsymbol{u} \in \mathbb{H}\left|u_{i}\right|_{\Omega^{\mathrm{e}}} \in P_{p}\left(\Omega^{\mathrm{e}}\right), \quad i \in\{1,2,3\}\right\}, \\
& \hat{V}_{2 h}^{p}=\left\{\boldsymbol{E} \in L^{2}(\Omega)\left|E_{i j}\right|_{\Omega^{\mathrm{e}}} \in P_{p}\left(\Omega^{\mathrm{e}}\right), \quad i, j \in\{1,2,3\}\right\}, \\
& \hat{V}_{3 h}^{p}=\left\{\psi \in L^{2}\left(\Sigma_{\mathrm{f}}\right)|\psi|_{\Sigma_{\mathrm{f}}^{\mathrm{e}}} \in P_{p}\left(\Sigma_{\mathrm{f}}^{\mathrm{e}}\right)\right\},
\end{aligned}
$$

as the solution space for the time-discretized problem. We then rewrite Problem 2 as a discretized coupling system with backward Euler finite differencing in time

Problem 3. Given $\left(\boldsymbol{u}_{h}^{(n-1)}, \boldsymbol{E}_{h}^{(n-1)}, \psi_{h}^{(n-1)}\right) \in \hat{V}_{1 h}^{p} \times \hat{V}_{2 h}^{p} \times \hat{V}_{3 h}^{p}$, find $\left(\boldsymbol{u}_{h}^{(n)}, \boldsymbol{v}_{h}^{(n)}, \boldsymbol{E}_{h}^{(n)}, \psi_{h}^{(n)}\right) \in$ $\hat{V}_{1 h}^{p} \times \hat{V}_{1 h}^{p} \times \hat{V}_{2 h}^{p} \times \hat{V}_{3 h}^{p}$, such that

$$
\begin{aligned}
& \sum_{\Omega^{\mathrm{e}}} \int_{\Omega^{\mathrm{e}}}\left(\rho_{h}^{0}\left(\frac{1}{\Delta t} \boldsymbol{v}_{h}^{(n)}+\boldsymbol{u}_{h}^{(n)} \cdot \boldsymbol{K}_{h}\right)+\gamma^{\mathrm{e}} \boldsymbol{v}_{h}^{(n)}\right) \cdot \boldsymbol{w}_{h} \mathrm{~d} \Omega+\sum_{\Omega^{\mathrm{e}}} \int_{\Omega^{\mathrm{e}}}\left(\boldsymbol{\Lambda}_{h}^{\boldsymbol{T}^{0}}: \boldsymbol{E}_{h}^{(n)}+\frac{\gamma^{\mathrm{e}}}{\Delta t} \boldsymbol{E}_{h}^{(n)}\right): \nabla \boldsymbol{w}_{h} \mathrm{~d} \Omega \\
& \quad+\sum_{\Sigma_{\mathrm{f}}^{\mathrm{e}}} \int_{\Sigma_{\mathrm{f}}^{\mathrm{e}}}\left(\left(\boldsymbol{\tau}_{\mathrm{f} h}^{(n)}-\sigma_{h}^{(n)} \boldsymbol{n}\right) \cdot \llbracket \boldsymbol{w}_{h} \rrbracket-\llbracket \boldsymbol{\tau}_{2 h}^{(n)} \cdot \boldsymbol{w}_{h} \rrbracket\right) \mathrm{d} \Sigma+\sum_{\Sigma_{\mathrm{f}}^{\mathrm{e}}} \alpha_{\mathrm{f}}^{\mathrm{e}} \int_{\Sigma_{\mathrm{f}}^{\mathrm{e}}} \llbracket \boldsymbol{n} \cdot\left(\boldsymbol{u}_{h}^{(n)}+\boldsymbol{v}_{h}^{(n)}\right) \rrbracket \llbracket \boldsymbol{n} \cdot \boldsymbol{w}_{h} \rrbracket \mathrm{d} \Sigma \\
& \quad+\sum_{\Sigma_{\mathrm{c}}^{\mathrm{e}}} \int_{\Sigma_{\mathrm{c}}^{\mathrm{e}}}\left(\left\{\left\{\boldsymbol{n} \cdot\left(\boldsymbol{\Lambda}_{h}^{\boldsymbol{T}^{0}}: \boldsymbol{E}_{h}^{(n)}\right)\right\}\right\}+\alpha_{\mathrm{c}}^{\mathrm{e}} \llbracket \boldsymbol{v}_{h}^{(n)} \rrbracket\right) \cdot \llbracket \boldsymbol{w}_{h} \rrbracket \mathrm{d} \Sigma \\
& =\frac{1}{\Delta t} \sum_{\Omega^{\mathrm{e}}} \int_{\Omega^{\mathrm{e}}}\left(\rho_{h}^{0} \boldsymbol{v}_{h}^{(n-1)} \cdot \boldsymbol{w}_{h}+\gamma^{\mathrm{e}} \boldsymbol{E}_{h}^{(n-1)}: \nabla \boldsymbol{w}_{h}\right) \mathrm{d} \Omega+\sum_{\Sigma_{\mathrm{f}}^{\mathrm{e}}} \int_{\Sigma_{\mathrm{f}}^{\mathrm{e}}}\left(\boldsymbol{n} \cdot\left(\boldsymbol{T}_{h}^{0}+\boldsymbol{T}_{h}^{\delta(n)}\right)\right) \cdot \llbracket \boldsymbol{w}_{h} \rrbracket \mathrm{d} \Sigma
\end{aligned}
$$

$$
\begin{gathered}
\sum_{\Omega^{\mathrm{e}}} \int_{\Omega^{\mathrm{e}}}\left(\frac{1}{\Delta t} \boldsymbol{E}_{h}^{(n)}: \boldsymbol{H}_{h}+\boldsymbol{v}_{h}^{(n)} \cdot\left(\nabla \cdot \boldsymbol{H}_{h}\right)\right) \mathrm{d} \Omega+\sum_{\Sigma_{\mathrm{f}}^{\mathrm{e}}} \int_{\Sigma_{\mathrm{f}}^{\mathrm{e}}}\left(\left\{\left\{\boldsymbol{v}_{h}^{(n)}\right\}\right\} \cdot \llbracket \boldsymbol{n} \cdot \boldsymbol{H}_{h} \rrbracket+\boldsymbol{s}_{h}^{(n)} \cdot\left\{\left\{\boldsymbol{n} \cdot \boldsymbol{H}_{h}\right\}\right\}\right) \mathrm{d} \Sigma \\
\quad+\sum_{\Sigma_{\mathrm{c}}^{\mathrm{e}}} \int_{\Sigma_{\mathrm{c}}^{\mathrm{e}}}\left(\left\{\left\{\boldsymbol{v}_{h}^{(n)}\right\}\right\}+\alpha_{\mathrm{c}}^{\mathrm{e}} \llbracket \boldsymbol{n} \cdot\left(\boldsymbol{\Lambda}_{h}^{\boldsymbol{T}^{0}}: \boldsymbol{E}_{h}^{(n)}\right) \rrbracket\right) \cdot \llbracket \boldsymbol{n} \cdot \boldsymbol{H}_{h} \rrbracket \mathrm{d} \Sigma=\frac{1}{\Delta t} \sum_{\Omega^{\mathrm{e}}} \int_{\Omega^{\mathrm{e}}} \boldsymbol{E}_{h}^{(n-1)}: \boldsymbol{H}_{h} \mathrm{~d} \Omega,
\end{gathered}
$$

$$
\int_{\Sigma_{\mathrm{f}}^{\mathrm{e}}} \psi_{h}^{(n)} \varphi_{h} \mathrm{~d} \Sigma+\Delta t \int_{\Sigma_{\mathrm{f}}^{\mathrm{e}}} \mathcal{G}\left(s_{h}^{(n)}, \psi_{h}^{(n)}\right) \varphi_{h} \mathrm{~d} \Sigma=\int_{\Sigma_{\mathrm{f}}^{\mathrm{e}}} \psi_{h}^{(n-1)} \varphi_{h} \mathrm{~d} \Sigma
$$


with

$$
\begin{gathered}
\boldsymbol{\tau}_{2 h}^{(n)}+\nabla^{\Sigma} \cdot\left(\boldsymbol{u}_{h}^{(n)}\left(\boldsymbol{n} \cdot \boldsymbol{T}_{h}^{0}\right)\right)=0, \\
\sigma_{h}^{(n)}+\boldsymbol{n} \cdot\left(\boldsymbol{n} \cdot\left\{\left\{\boldsymbol{\Lambda}_{h}^{\boldsymbol{T}^{0}}: \boldsymbol{E}_{h}^{(n)}\right\}\right\}+\left\{\left\{\boldsymbol{\tau}_{2 h}^{(n)}\right\}\right\}\right)=-\boldsymbol{n} \cdot\left(\boldsymbol{T}_{h}^{0}+\boldsymbol{T}_{h}^{\delta(n)}\right) \cdot \boldsymbol{n}, \\
\boldsymbol{u}^{(n)}-\Delta t \boldsymbol{v}^{(n)}=\boldsymbol{u}^{(n-1)}, \\
\boldsymbol{s}_{h}^{(n)}-\llbracket \boldsymbol{v}_{h \|}^{(n)} \rrbracket=0, \quad s_{h}^{(n)}=\left|\boldsymbol{s}_{h}^{(n)}\right|, \\
\boldsymbol{s}_{h}^{(n)} \mathcal{F}\left(\sigma_{h}^{(n)}, s_{h}^{(n)}, \psi_{h}^{(n)}\right)-s_{h}^{(n)} \boldsymbol{\tau}_{\mathrm{f} h}^{(n)}=0,
\end{gathered}
$$

for all test functions $\left(\boldsymbol{H}_{h}, \boldsymbol{w}_{h}, \varphi_{h}\right) \in \hat{V}_{1 h}^{p} \times \hat{V}_{2 h}^{p} \times \hat{V}_{3 h}^{p}$.

REMARK 1. We choose an implicit Euler discretization rather than a higher-order scheme for the coupled system due to the fact that the solution of the time-continuous Problem 1 has limited regularity in time, as is apparent in the solution space (cf.(2.19)). In particular, the particle velocity, $\boldsymbol{v}$, is only once differentiable in the weak sense. A detailed proof justifying the solution space can be found in our companion paper [66]. Numerical examples in Section 5 illustrate that even with the highest refinements considered in both space and time, some oscillations remain in the solutions.

The coupling system described by Problem 3 can be solved by a general nonlinear optimization approach such as Newton's method. This approach is computationally expensive, however, because of the factorization of global Hessian matrices. We propose a computationally efficient iterative scheme as an alternative in the next section.

4. Iterative coupling and multi-rate time scheme. In order to obtain an accurate solution with affordable effort, we derive an alternative approach using a fixed-point iteration while separating the state ODE from the elastic wave equation, and conducting domain decomposition (e.g. [9, Section 6.1]) to separate the variables on $\Sigma_{\mathrm{f}}^{\mathrm{e}}$ from elsewhere.

We rewrite $(3.4 \mathrm{a}, \mathrm{b})$ by moving the surface integration terms on $\Sigma_{\mathrm{c}}^{\mathrm{e}}$ to the right-hand-side, and construct a sequence of linear-nonlinear coupling problems for each time step $\left[t_{n-1}, t_{n}\right]$, which follows the iteration for $k=1,2, \cdots$, with $v^{(n, k)}$ representing the value at the $k^{\text {th }}$ iteration of a variable $v$ at time $t=t_{n}$. Then, we seek the solution of the following

Problem 4. Given $\left(\boldsymbol{u}_{h}^{(n-1)}, \boldsymbol{E}_{h}^{(n-1)}, \psi_{h}^{(n-1)}\right) \in \hat{V}_{1 h}^{p} \times \hat{V}_{2 h}^{p} \times \hat{V}_{3 h}^{p}$, and $\left(\boldsymbol{v}_{h}^{(n, k-1)}, \boldsymbol{E}_{h}^{(n, k-1)}\right) \in \hat{V}_{1 h}^{p} \times$ 
$\hat{V}_{2 h}^{p}$, find $\left(\boldsymbol{u}_{h}^{(n, k)}, \boldsymbol{v}_{h}^{(n, k)}, \boldsymbol{E}_{h}^{(n, k)}, \psi_{h}^{(n, k)}\right) \in \hat{V}_{1 h}^{p} \times \hat{V}_{1 h}^{p} \times \hat{V}_{2 h}^{p} \times \hat{V}_{3 h}^{p}$, such that

$$
\begin{aligned}
\sum_{\Omega^{\mathrm{e}}} \int_{\Omega^{\mathrm{e}}} & \left(\rho_{h}^{0}\left(\frac{1}{\Delta t} \boldsymbol{v}_{h}^{(n, k)}+\boldsymbol{u}_{h}^{(n, k)} \cdot \boldsymbol{K}_{h}\right)+\gamma^{\mathrm{e}} \boldsymbol{v}_{h}^{(n)}\right) \cdot \boldsymbol{w}_{h} \mathrm{~d} \Omega+\sum_{\Omega^{\mathrm{e}}} \int_{\Omega^{\mathrm{e}}}\left(\boldsymbol{\Lambda}_{h}^{\boldsymbol{T}^{0}}: \boldsymbol{E}_{h}^{(n, k)}+\frac{\gamma^{\mathrm{e}}}{\Delta t} \boldsymbol{E}_{h}^{(n, k)}\right): \nabla \boldsymbol{w}_{h} \mathrm{~d} \Omega \\
& +\sum_{\Sigma_{\mathrm{f}}^{\mathrm{e}}} \int_{\Sigma_{\mathrm{f}}^{\mathrm{e}}}\left(\left(\boldsymbol{\tau}_{\mathrm{f} h}^{(n, k)}-\sigma_{h}^{(n, k)} \boldsymbol{n}\right) \cdot \llbracket \boldsymbol{w}_{h} \rrbracket-\llbracket \boldsymbol{\tau}_{2 h}^{(n, k)} \cdot \boldsymbol{w}_{h} \rrbracket\right) \mathrm{d} \Sigma \\
& +\sum_{\Sigma_{\mathrm{f}}^{\mathrm{e}}} \alpha_{\mathrm{f}}^{\mathrm{e}} \int_{\Sigma_{\mathrm{f}}^{\mathrm{e}}}\left(\llbracket \boldsymbol{n} \cdot\left(\boldsymbol{u}_{h}^{(n, k)}+\boldsymbol{v}_{h}^{(n, k)}\right) \rrbracket \llbracket \boldsymbol{n} \cdot \boldsymbol{w}_{h} \rrbracket\right) \mathrm{d} \Sigma \\
= & \frac{1}{\Delta t} \sum_{\Omega^{\mathrm{e}}} \int_{\Omega^{\mathrm{e}}}\left(\rho_{h}^{0} \boldsymbol{v}_{h}^{(n-1)} \cdot \boldsymbol{w}_{h}+\gamma^{\mathrm{e}} \boldsymbol{E}_{h}^{(n-1)}: \nabla \boldsymbol{w}_{h}\right) \mathrm{d} \Omega \\
& -\sum_{\Sigma_{\mathrm{c}}^{\mathrm{e}}} \int_{\Sigma_{\mathrm{c}}^{\mathrm{e}}}\left(\left\{\left\{\boldsymbol{n} \cdot\left(\boldsymbol{\Lambda}_{h}^{\boldsymbol{T}^{0}}: \boldsymbol{E}_{h}^{(n, k-1)}\right)\right\}\right\}+\alpha_{\mathrm{c}}^{\mathrm{e}} \llbracket \boldsymbol{v}_{h}^{(n, k-1)} \rrbracket\right) \cdot \llbracket \boldsymbol{w}_{h} \rrbracket \mathrm{d} \Sigma \\
& +\sum_{\Sigma_{\mathrm{f}}^{\mathrm{e}}} \int_{\Sigma_{\mathrm{f}}^{\mathrm{e}}}\left(\boldsymbol{n} \cdot\left(\boldsymbol{T}_{h}^{0}+\boldsymbol{T}_{h}^{\delta(n)}\right)\right) \cdot \llbracket \boldsymbol{w}_{h} \rrbracket \mathrm{d} \Sigma,
\end{aligned}
$$

(4.1b)

$$
\begin{gathered}
\sum_{\Omega^{\mathrm{e}}} \int_{\Omega^{\mathrm{e}}}\left(\frac{1}{\Delta t} \boldsymbol{E}_{h}^{(n, k)}: \boldsymbol{H}_{h}+\boldsymbol{v}_{h}^{(n, k)} \cdot\left(\nabla \cdot \boldsymbol{H}_{h}\right)\right) \mathrm{d} \Omega+\sum_{\Sigma_{\mathrm{f}}^{\mathrm{e}}} \int_{\Sigma_{\mathrm{f}}^{\mathrm{e}}}\left(\left\{\left\{\boldsymbol{v}_{h}^{(n, k)}\right\}\right\} \cdot \llbracket \boldsymbol{n} \cdot \boldsymbol{H}_{h} \rrbracket+\boldsymbol{s}_{h}^{(n, k)} \cdot\left\{\left\{\boldsymbol{n} \cdot \boldsymbol{H}_{h}\right\}\right\}\right) \mathrm{d} \Sigma \\
=\frac{1}{\Delta t} \sum_{\Omega^{\mathrm{e}}} \int_{\Omega^{\mathrm{e}}} \boldsymbol{E}_{h}^{(n-1)}: \boldsymbol{H}_{h} \mathrm{~d} \Omega-\sum_{\Sigma_{\mathrm{c}}^{\mathrm{e}}} \int_{\Sigma_{\mathrm{c}}^{\mathrm{e}}}\left(\left\{\left\{\boldsymbol{v}_{h}^{(n, k-1)}\right\}\right\}+\alpha_{\mathrm{c}}^{\mathrm{e}} \llbracket \boldsymbol{n} \cdot\left(\boldsymbol{\Lambda}_{h}^{\boldsymbol{T}^{0}}: \boldsymbol{E}_{h}^{(n, k-1)}\right) \rrbracket\right) \cdot \llbracket \boldsymbol{n} \cdot \boldsymbol{H}_{h} \rrbracket \mathrm{d} \Sigma
\end{gathered},
$$

$$
\int_{\Sigma_{\mathrm{f}}^{\mathrm{e}}} \psi_{h}^{(n, k)} \varphi_{h} \mathrm{~d} \Sigma+\Delta t \int_{\Sigma_{\mathrm{f}}^{\mathrm{e}}} \mathcal{G}\left(s_{h}^{(n, k)}, \psi_{h}^{(n, k)}\right) \varphi_{h} \mathrm{~d} \Sigma=\int_{\Sigma_{\mathrm{f}}^{\mathrm{e}}} \psi_{h}^{(n-1)} \varphi_{h} \mathrm{~d} \Sigma,
$$

with

$$
\begin{aligned}
& \boldsymbol{\tau}_{2 h}^{(n, k)}+\nabla^{\Sigma} \cdot\left(\boldsymbol{u}_{h}^{(n, k)}\left(\boldsymbol{n} \cdot \boldsymbol{T}_{h}^{0}\right)\right)=0, \quad \sigma_{h}^{(n, k)}+\boldsymbol{n} \cdot\left(\boldsymbol{n} \cdot\left\{\left\{\boldsymbol{\Lambda}_{h}^{\boldsymbol{T}^{0}}: \boldsymbol{E}_{h}^{(n, k)}\right\}\right\}+\left\{\left\{\boldsymbol{\tau}_{2 h}^{(n, k)}\right\}\right\}\right)=-\boldsymbol{n} \cdot\left(\boldsymbol{T}_{h}^{0}+\boldsymbol{T}_{h}^{\delta(n)}\right) \cdot \boldsymbol{n}, \\
& \boldsymbol{u}_{h}^{(n, k)}-\Delta t \boldsymbol{v}_{h}^{(n, k)}=\boldsymbol{u}_{h}^{(n-1)}, \quad \boldsymbol{s}_{h}^{(n, k)}-\llbracket \boldsymbol{v}_{h \|}^{(n, k)} \rrbracket=0, \quad s_{h}^{(n, k)}=\left|\boldsymbol{s}_{h}^{(n, k)}\right|
\end{aligned}
$$

$\boldsymbol{s}_{h}^{(n, k)} \mathcal{F}\left(\sigma_{h}^{(n, k)}, s_{h}^{(n, k)}, \psi_{h}^{(n, k)}\right)-s_{h}^{(n, k)} \boldsymbol{\tau}_{\mathrm{f} h}^{(n, k)}=0$,

for all test functions $\left(\boldsymbol{H}_{h}, \boldsymbol{w}_{h}, \varphi_{h}\right) \in \hat{V}_{1 h}^{p} \times \hat{V}_{2 h}^{p} \times \hat{V}_{3 h}^{p}$,

For the first iteration $k=1$ the initial value of variables are obtained from the previous time step by

$$
\boldsymbol{v}_{h}^{(t, 0)}=\boldsymbol{v}_{h}^{(n-1)}, \quad \boldsymbol{u}_{h}^{(t, 0)}=\boldsymbol{u}_{h}^{(n-1)}, \quad \boldsymbol{E}_{h}^{(t, 0)}=\boldsymbol{E}_{h}^{(n-1)}, \quad \psi_{h}^{(t, 0)}=\psi_{h}^{(n-1)} .
$$

We show the contraction of this iterative algorithm in B with criteria for $\gamma^{\mathrm{e}}, \alpha_{\mathrm{f}}^{\mathrm{e}}, \alpha_{\mathrm{c}}^{\mathrm{e}}$ and $\Delta t$ precisely given in Theorem B.1.

Due to the fact that the evolution of rupture state is a significantly faster physical process than the wave propagation and scattering during fault propagation, we use a multi-rate scheme, 
which is based on the same backward Euler time integration method but with finer substeps. In particular, as an alternative to (4.1c), we use $N$ substeps within each time step $\Delta t$, which generates the multi-rate scheme by

$$
\begin{aligned}
& \int_{\Sigma_{\mathrm{f}}^{\mathrm{e}}} \psi_{h}^{(n, k)} \varphi_{h} \mathrm{~d} \Sigma+\frac{\Delta t}{N} \sum_{i=1}^{N} \int_{\Sigma_{\mathrm{f}}^{\mathrm{e}}} \theta_{h}^{(n), i} \varphi_{h} \mathrm{~d} \Sigma=\int_{\Sigma_{\mathrm{f}}^{\mathrm{e}}} \psi_{h}^{(n-1)} \varphi_{h} \mathrm{~d} \Sigma, \\
& \theta_{h}^{(n), i}=-\mathcal{G}\left(s_{h}^{(n-1)}+\frac{i}{N}\left(s_{h}^{(n, k)}-s_{h}^{(n-1)}\right), \psi_{h}^{(n-1)}+\frac{\Delta t}{N} \sum_{j=1}^{i} \theta_{h}^{(n), j}\right) .
\end{aligned}
$$

We solve the coupled nonlinear problem (4.1a-f) by defining a constrained optimization problem, in which the objective function,

$$
\mathfrak{L}:=\frac{1}{2}\left\|\frac{\boldsymbol{s}_{h}^{(n, k)}}{s_{h}^{(n, k)}} \mathcal{F}\left(\sigma_{h}^{(n, k)}, s_{h}^{(n, k)}, \psi_{h}^{(n, k)}\right)-\boldsymbol{\tau}_{\mathrm{f} h}^{(n, k)}\right\|^{2},
$$

follows from (4.2b) upon normalization, with linear constraints (4.1a,b) and (4.2a), and nonlinear constraint (4.1c). Compared with the original, implicitly discretized problem, the iterative problem is localized to each element, where the Hessian matrices become block-diagonal. Details of the numerical algorithm solving this problem using the Gauss-Newton method are provided in C.

5. Computational experiments. Existing results demonstrate the complex features in the nucleation of ruptures, especially with dissimilar materials $(e . g .[6,13])$ and non-planar faults with geometries such as corners or branches $(e . g .[46,30,38])$. In both cases, slip-pulse responses may apear in the simulations. Ill-posedness without regularization has been apparent for a wide range of material contrasts (for example, in [13]), that is, the numerical solution does not converge under mesh refinements. In this section, we carry out computational experiments with our algorithm including regularization by artificial viscosity, which yields convergent results. To benchmark with existing results, we impose the identical assumption that the effects of the initial gravitational potential and prestress on the elastic wave propagation are ignored ("non-prestressed") although prestress is still accounted for in the boundary source term. In other words, $\Lambda_{i j k l}^{\boldsymbol{T}^{0}} \equiv C_{i j k l}$ and $\boldsymbol{\tau}_{2} \equiv 0$ in Subsections 5.1-5.5. We discuss the effects of static self-gravitation and prestress on the solution in Subsection 5.6.

5.1. Planar fault with homogeneous material. We verify our numerical algorithm by testing it on benchmark problem "TPV102" designed by the SCEC/USGS Spontaneous Rupture Code Verification Project (SRCVP) [27], which has been used in recent dynamic rupture studies (e.g. [22]). The model is of size $[-18 \mathrm{~km}, 18 \mathrm{~km}] \times[-18 \mathrm{~km}, 0 \mathrm{~km}] \times[-12 \mathrm{~km}, 12 \mathrm{~km}]$, where the depth is along the $x_{2}$-axis, and $x_{2}=0$ represents the earth's surface at which a traction-free boundary condition is applied. The planar strike-slip rupture is located on $x_{3}=0$, on which the friction parameters are set to be slip-weakening within the central portion $[-15 \mathrm{~km}, 15 \mathrm{~km}] \times[-15 \mathrm{~km}, 0 \mathrm{~km}]$, with a smooth transition into a slip-strengthening condition at positions close to the boundary of the model. The nonlinear dependency of friction magnitude upon normal stress, slip rate and state variable is, here, given by

$$
\mathcal{F}(\sigma, s, \psi)=a \sigma \operatorname{arcsinh}\left(\frac{s}{2 s_{0}} \exp \left(\frac{f_{0}+b \ln \left(s_{0} \psi / L\right)}{a}\right)\right),
$$

while the state ODE is given by

$$
\mathcal{G}(s, \psi)=-1+\frac{s}{L} \psi
$$

The material parameters and components of the prestress tensor are shown in Table 5.1, where the coefficient $a$ as well as the initial value of the state variable are assigned by a function depending on position, and satisfy the quasi-static assumptions. 


\begin{tabular}{|c|c|c|c|}
\hline$V_{p}$ & $V_{s}$ & $\rho$ & $\psi_{\text {ini }}$ \\
\hline $6.0 \mathrm{~km} / \mathrm{s}$ & $3.464 \mathrm{~km} / \mathrm{s}$ & $2.67 \mathrm{~g} / \mathrm{cm}^{3}$ & $1.606 \times 10^{9 \sim 13} \mathrm{~s}$ \\
\hline \hline$a$ & $b$ & $L$ & $s_{0}$ \\
\hline $0.008 \sim 0.016$ & 0.012 & $2 \mathrm{~cm}$ & $1 \mu \mathrm{m} / \mathrm{s}$ \\
\hline \hline$f_{0}$ & $s_{c}$ & $\left(\boldsymbol{T}_{0}\right)_{13}$ & $\left(\boldsymbol{T}_{0}\right)_{33}$ \\
\hline 0.6 & $10^{-6} \mu \mathrm{m} / \mathrm{s}$ & $75 \mathrm{MPa}$ & $-120 \mathrm{MPa}$ \\
\hline
\end{tabular}

Table 5.1: Material parameters, rupture coefficients and prestress in the homogeneous-elastic planar rupture model TPV102. The components of $\boldsymbol{T}_{0}$ not listed take the value 0 . The quantity $s_{c}$ is an aseismic (creeping) velocity that keeps $s$ away from 0 .

The nucleation of rupture takes place with a time-variant perturbation in stress, $\boldsymbol{T}^{\delta}$, in a ball-shaped region centered at $(0.0 \mathrm{~km},-7.5 \mathrm{~km}, 0.0 \mathrm{~km})$ with radius $r_{0}=3 \mathrm{~km}$, following the scalar function

$$
\begin{aligned}
T_{13}^{\delta}(r, t) & =T_{31}^{\delta}(r, t)=\tau^{\delta} g(r) h(t-0), \\
g(r) & =\left\{\begin{array}{ll}
\exp \left(\frac{r^{2}}{r^{2}-r_{0}^{2}}\right) & \text { if } r<r_{0} \\
0 & \text { if } r \geq r_{0}
\end{array} \quad, \quad h(t)= \begin{cases}\exp \left(\frac{(t-1)^{2}}{t(t-2)}\right) & \text { if } 0<t<1 \\
1 & \text { if } t \geq 1\end{cases} \right.
\end{aligned}
$$

where $r$ is the distance of a point in the model to the hypocenter, and $\tau^{\delta}=25 \mathrm{MPa}$.

We extend the model to $[-20 \mathrm{~km}, 20 \mathrm{~km}] \times[-20 \mathrm{~km}, 0 \mathrm{~km}] \times[-12 \mathrm{~km}, 12 \mathrm{~km}]$, with extra layers for absorbing boundaries, and discretize the computational domain using a fully unstructured tetrahedral mesh with 1,912,556 elements generated with DistMesh [44] and Tetgen [54]. The rupture plane is properly aligned by subdomain interfaces, and the triangular facets on the fault have a mean area of $0.015 \mathrm{~km}^{2}$, as is shown in Figure 1. In the numerical simulations we use polynomial orders $p=1, p=2$ and $p=3$. The viscosity coefficient is assigned elementwise, which takes a constant value of $1.0 \times 10^{-7} \mathrm{GPa} \cdot \mathrm{s}$ within the elements attached to the rupture plane, and 0 in the remaining ones. The time steps used for $p=1,2,3$ are $0.064,0.032$ and $0.016 \mathrm{~ms}$, respectively, and each step is divided evenly into 16 substeps (see (4.4ab)) to solve the state ODE. We conducted a domain decomposition and ran the simulation on a distributed memory machine using 256 cores. We show the snapshots at $\mathrm{t}=4.5,5.5$ and $6.5 \mathrm{~s}$ for the $p=2$ simulation, with the three components of particle velocity in the volume listed in Figure 2. Figure 3 shows the rupture contour. The scattering of black dots is due to hard thresholding of the slip-rate at $1 \mathrm{~mm} / \mathrm{s}$, which illustrates the oscillatory behavior of the solution. The rupture propagation, and the time variations of friction force, normal stress, as well as state variable are also shown in Figure 4.

We benchmark our numerical result with the ones using a spectral element (SE) method ([32]) and a finite element (FE) method (PyLith [2]), by comparing the seismograms of stations located on the fault plane as well as the earth's surface, as is shown in Figures 5 and 6, respectively. Clearly, all the physical quantities obtained from the DG simulations match the reference data produced by existing software within apt tolerance, even with a coarser mesh compared with the ones used by FE or SE (both using a semi-regular mesh with a size of $0.1 \mathrm{~km}$, and the SE modeling using $p=5$ ). The numerical results obtained by the SE method and our DG method with $p \geq 2$ show very good agreement. In general, numerical results generated by lower-order schemes (FE, DG with $p=1$ ) show slightly slower propagation speeds of rupture. This effect can be intuitively related to the intrinsic dissipation of the numerical methods, which affects the solution in a similar manner 


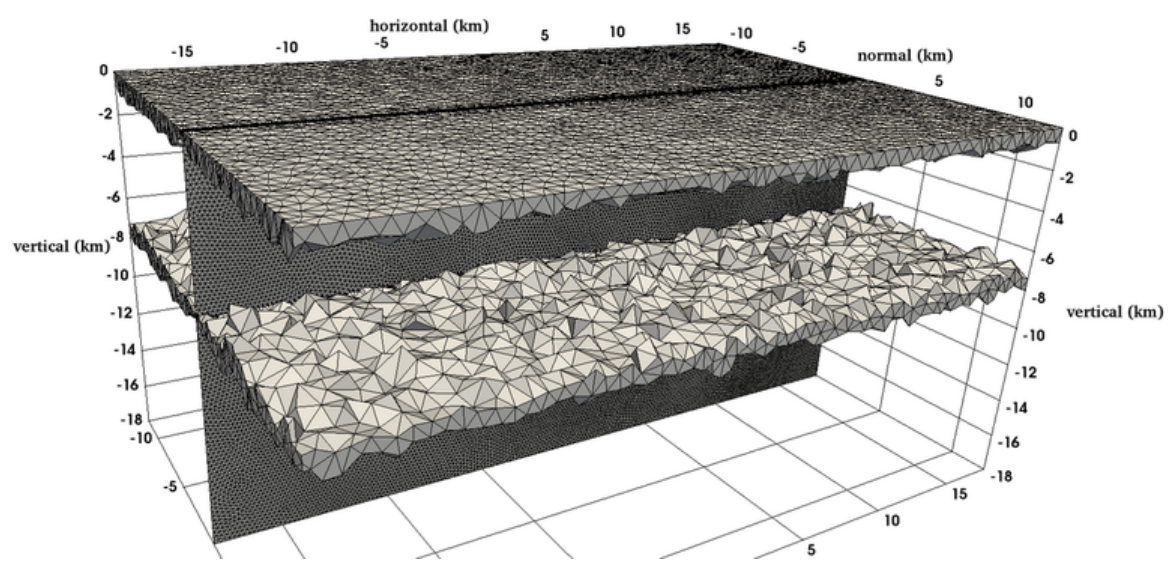

Fig. 1: Visualization of "TPV102" model with unstructured tetrahedral mesh,

as artificial viscosity (see also discussions in Subsection 5.5). For higher-order schemes with smaller numerical dissipation and artificial viscosity, and correspondingly smaller time steps required by stability conditions, the numerical solutions approach one with relatively fast rupture propagation speed, which is consistent with the physics (see Figures 5 and 6).

5.2. Convergence test on a homogeneous planar fault. In the absence of a closed-form solution for the rate- and state-dependent friction laws in dimension 3, we show the convergence of our DG algorithm with refinements in space and time discretizations to a reference solution. We consider a planar fault in a homogeneous surrounding medium. We reduce the size of the "TPV102" model to a rectangular box of size $[-6 \mathrm{~km}, 6 \mathrm{~km}] \times[-12 \mathrm{~km}, 0 \mathrm{~km}] \times[-6 \mathrm{~km}, 6 \mathrm{~km}]$, and keep the free surface on the top of the box at $x_{2}=0$. Absorbing layers (1.8km in thickness) are placed on the remaining sides of the box. We generate a set of semi-regular tetrahedral meshes that are gradually refined when getting closer to the rupture plane, such that the elements attached to the rupture plane are uniform in size. Their identical inradius is considered to be the mesh size in Figure 8, illustrating the convergence. The elastic parameters of the material and components of the prestress tensor are identical to those shown in Table 5.1, where coefficient " $a$ " as well as the initial value of the state variable are assigned such that the rupture gets strengthened when approaching the absorbing boundaries, in the same manner as in "TPV102".

In Figure 7, we show one of the meshes and rupture parameters used in this convergence test. To make the problem sufficiently regular for the convergence test, we choose the viscosity coefficient equal to $\gamma=2.0 \times 10^{-5} \mathrm{GPa} \cdot \mathrm{s}$, which is larger than the one in the "TPV102" test; this has little impact on the propagation of rupture (see also Section 5.5). For the convergence test with spatial $h p$-refinements, we use a uniform sufficiently small time step of $\Delta t=0.02 \mathrm{~ms}$ without multi-rate, for the tests on 6 different mesh sizes and $1-4$ polynomial orders. We estimate the numerical error by analyzing the slip curves recorded at 25 on-fault receivers (Figure 7 ). The numerical results obtained from the finest mesh with 4th order polynomial basis are considered as the reference, and the errors for the other mesh-size and polynomial orders are computed as the sum of $L^{2}$ norms of the differences over all 25 receivers. The curve plotted in Figure 8(a) shows convergence rates of approximately $p+1$ for polynomial orders $p=1$ and 2 , which are consistent with the optimal rates of the DG method. However, we cannot obtain a higher convergence rate even with increasing order due to the limited regularity of the solution to the coupled problem. Meanwhile, despite the fact that no theoretical convergence rate in time can be obtained (the particle velocity is up to first order weakly differentiable in time), we nevertheless obtain numerical convergence for this particular example, with fixed mesh size $h=12.0 \mathrm{~m}$ and $p=2$ polynomial basis. The errors are 


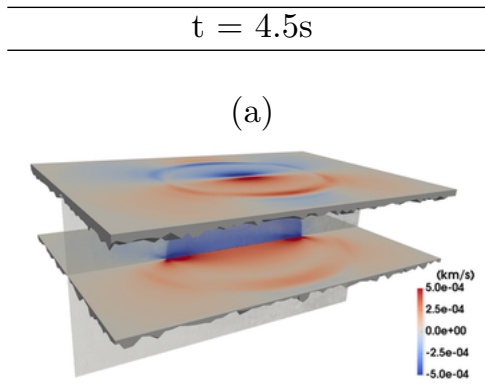

(d)

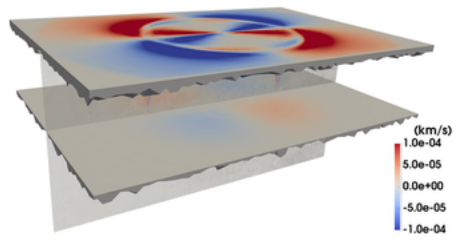

$(\mathrm{g})$

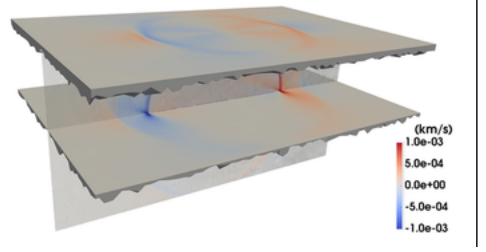

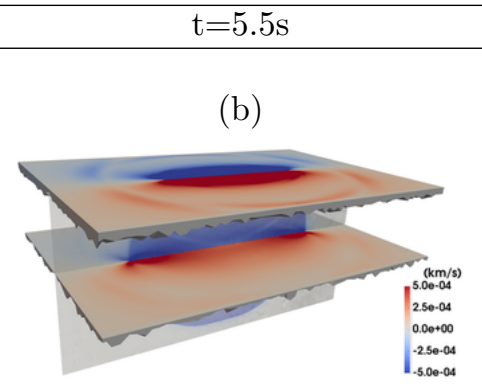

(e)

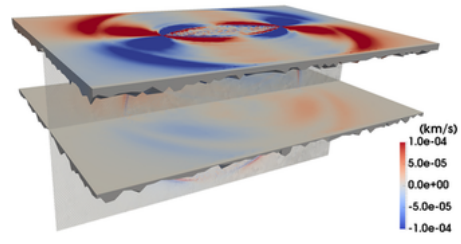

(h)

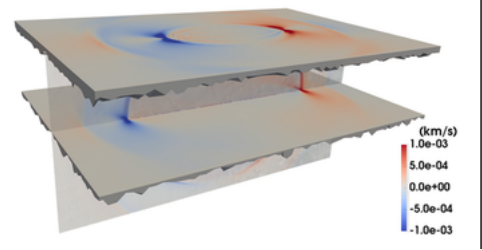

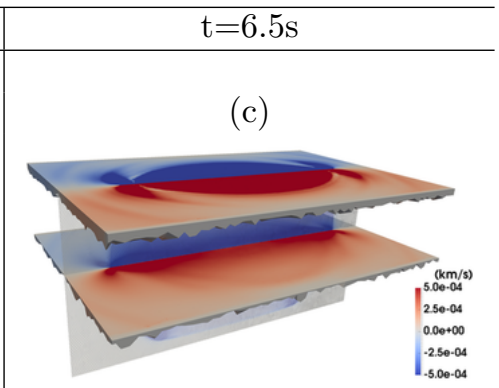

(f)

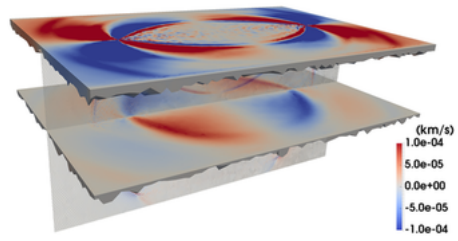

(i)

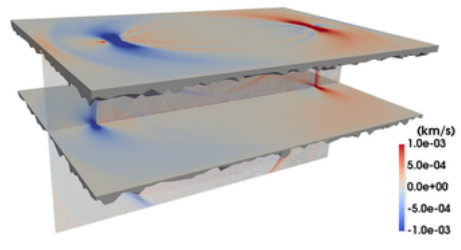

Fig. 2: Snapshots of particle velocities for "TPV102" model at $\mathrm{t}=4.5,5.5,6.5 \mathrm{~s}$ with $(\mathrm{a}, \mathrm{b}, \mathrm{c})$ horizontal component, (d, e, f) vertical component, (g, h, i) normal component, computed by DG method with polynomial order 2 .

computed based on the finest time step $\Delta t=0.02 \mathrm{~ms}$ and highest multi-rate (evenly subdividing $\Delta t$ into 64 steps), which is shown in Figure 8(b). The convergence in time is close to first order, and the multi-rate scheme can reduce the numerical error with roughly the same rate.

5.3. Planar fault with a bi-material. The presence of a bi-material rupture interface breaks the symmetry of stress across the fault surface. The strongest coupling at the rupture tip brings significant impact on the propagation of rupture front, regardless of specific properties of friction law $[52,4,53]$. To show the capability of our algorithm to simulate a bi-material dynamic rupture problem, we modify the strong contrast bi-material model "TPV6" designed by SCEC/USGS SRCVP, by replacing the linear slip-weakening friction law with the rate- and state-dependent friction law given in Section 5.1. The material parameters are listed in Table 5.2. The size of the model is $[-18 \mathrm{~km}, 18 \mathrm{~km}] \times[-18 \mathrm{~km}, 0 \mathrm{~km}] \times[-10 \mathrm{~km}, 10 \mathrm{~km}]$. The location of the rupture and the free surface, as well as the space-time dependency of stress perturbation $\boldsymbol{T}^{\delta}$ are the same as for the "TPV102" model in Section 5.1. For the sake of computational efficiency, we discretize the model using a quasi-regular tetrahedral mesh with 1,058,400 elements, which is also locally refined, and the fault plane is decomposed into uniform triangles with an area of $1.125 \times 10^{-2} \mathrm{~km}^{2}$, as is shown in Figure 9. We also construct a finer mesh with 1,617,408 elements, and on the fault plane the uniform triangles with an area of $7.812 \times 10^{-3} \mathrm{~km}^{2}$.

In the numerical simulation we use polynomial orders $p=1$ and $p=2$, and compute the wavefields until $t=15$.0s. We assign elementwise a constant viscosity coefficient, equal to $2.0 \times$ $10^{-4} \mathrm{GPa} \cdot \mathrm{s}$ in the elements attached to the rupture plane, and 0 in the remaining ones. We use time steps of $0.05 \mathrm{~ms}$ and $0.025 \mathrm{~ms}$, respectively, for the coarser mesh with $p=1$ and $p=2$, and $0.032 \mathrm{~ms}$ 


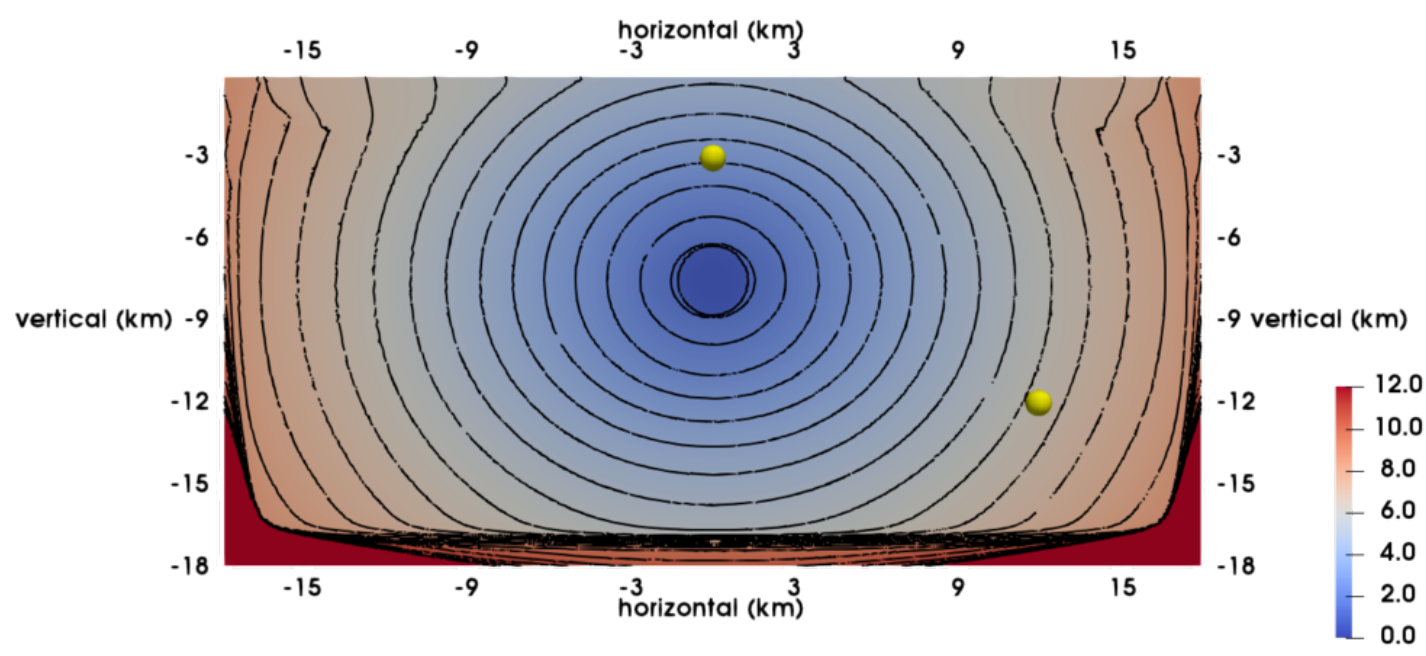

Fig. 3: Rupture contour plot (showing the propagation time when slip-rate exceeds $1 \mathrm{~mm} / \mathrm{s}$ ) of the "TPV102" model, with interval step of $0.5 \mathrm{~s}$. Balls in the figure are indicating the location of stations whose seismograms are plotted in Figure 5.

for the finer mesh with $p=1$. For the state ODE we use a multi-rate scheme with 16 substeps. We visualize the numerical result of the $p=2$ simulation in Figures 10-12. Figure 10 shows snapshots at $t=5.0,6.0$ and 7.0s, with the three components of particle velocity in the volume listed in (a)-(i). Figure 11 shows the rupture contour. Compared with Figure 3, the scattering of black dots stands out on the slower propagating side, which illustrates the much more oscillatory behavior of the bi-material case than the homogeneous case. Spatial hp-refinements mitigate this (reducing the magnitude while increasing the frequency of oscillation, see Figures 13-14 for example), while further time refinement has no observable effect.

The slip rate, friction force, normal stress as well as state variable as functions of time are shown in Figure 12. In these figures, we observe the asymmetric propagation speed of the rupture, which is consistent with other results in the study of bi-material models. We show a comparison of seismograms generated by different mesh sizes and polynomial orders, for deep on-fault stations in Figure 13, for shallow on-fault stations in Figure 14 where we note the interaction with the free surface, and for on-ground stations in Figure 15. These demonstrate the convergence of our algorithm with $h p$-refinement. The effect of refinement is much more pronounced on the slower propagating side. Furthermore, the difference among seismograms is much more significant than this difference in the homogeneous test example. This observation can be closely related to the nonlinear coupling with normal stress varying in time.

5.4. A non-planar fault with homogeneous material. A realistic fault commonly has complex geometries, with bending, a step-over, and branching. Here, we consider two step-over faults with an offset of $1.5 \mathrm{~km}$, connected by a third fault, forming dihedral angles of $166^{\circ}$. The material parameters are chosen to be almost the same as in the "TPV102" model, except for the components of the prestress tensor, and are listed in Table 5.3; the state variable is computed based on the quasi-static assumption. The size of the model is $[-20 \mathrm{~km}, 20 \mathrm{~km}] \times[-20 \mathrm{~km}, 0 \mathrm{~km}] \times[-$ $12 \mathrm{~km}, 12 \mathrm{~km}]$. The space-time dependency of stress perturbation, $\boldsymbol{T}^{\delta}$, is mostly the same as in the "TPV102" model in Section 5.1, except that the hypocenter is placed at $(-9.0 \mathrm{~km},-7.5 \mathrm{~km}, 0.0 \mathrm{~km})$.

We discretize the model using a fully unstructured, and sufficiently refined, tetrahedral mesh 


$$
\mathrm{t}=4.5 \mathrm{~s} \quad \mathrm{t}=5.5 \mathrm{~s} \quad \mathrm{t}=6.5 \mathrm{~s}
$$
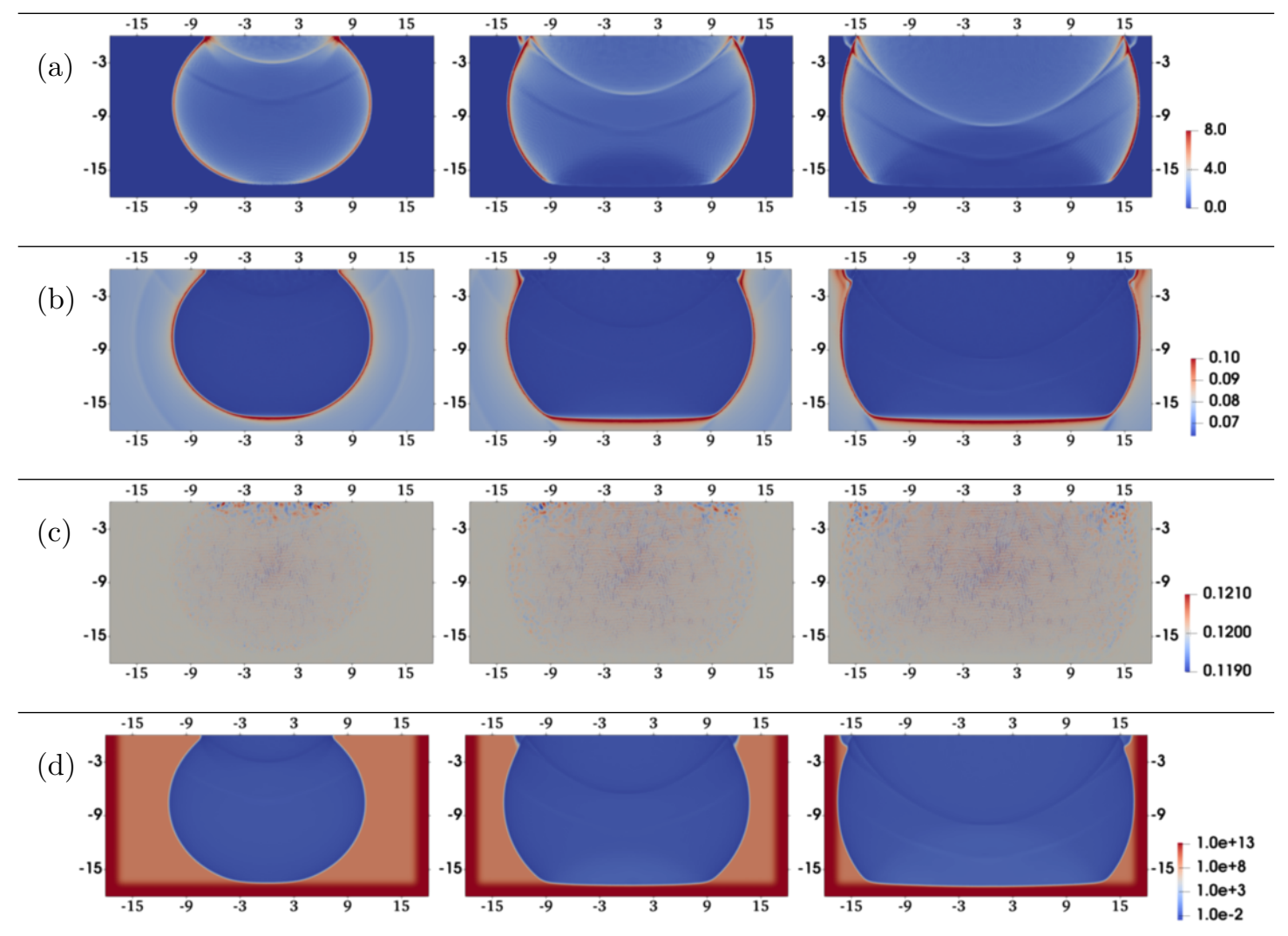

Fig. 4: Visualization on the rupture plane of "TPV102" model with (a) the slip rate with unit of $\mathrm{m} / \mathrm{s}$, (b) the magnitude of friction force with unit of $\mathrm{GPa}$, (c) the compressive normal stress with unit of $\mathrm{GPa},(\mathrm{d})$ the state variable ("age" of rupture) with unit of second, at time $\mathrm{t}=5.5,6.5,7.5$ s.

\begin{tabular}{|c|c|c|c|c|}
\hline$V_{p 1}$ & $V_{s 1}$ & $\rho_{1}$ & $V_{p 2}$ & $V_{s 2}$ \\
\hline $3.750 \mathrm{~km} / \mathrm{s}$ & $2.165 \mathrm{~km} / \mathrm{s}$ & $2.225 \mathrm{~g} / \mathrm{cm}^{3}$ & $6.0 \mathrm{~km} / \mathrm{s}$ & $3.464 \mathrm{~km} / \mathrm{s}$ \\
\hline \hline$\rho_{2}$ & $\psi_{\text {ini }}$ & $a$ & $b$ & $L$ \\
\hline $2.67 \mathrm{~g} / \mathrm{cm}^{3}$ & $1.606 \times 10^{9 \sim 13} \mathrm{~s}$ & $0.008 \sim 0.016$ & 0.012 & $2 \mathrm{~cm}$ \\
\hline \hline$s_{0}$ & $f_{0}$ & $s_{c}$ & $\left(\boldsymbol{T}_{0}\right)_{13}$ & $\left(\boldsymbol{T}_{0}\right)_{33}$ \\
\hline $1 \mu \mathrm{m} / \mathrm{s}$ & 0.6 & $10^{-6} \mu \mathrm{m} / \mathrm{s}$ & $75 \mathrm{MPa}$ & $-120 \mathrm{MPa}$ \\
\hline
\end{tabular}

Table 5.2: Material parameters, rupture coefficients and prestress in the modified bi-material model with planar rupture. The components of $\boldsymbol{T}_{0}$ not listed take the value 0 . The quantity $s_{c}$ is an aseismic (creeping) velocity that keeps $s$ away from 0. 
(a)
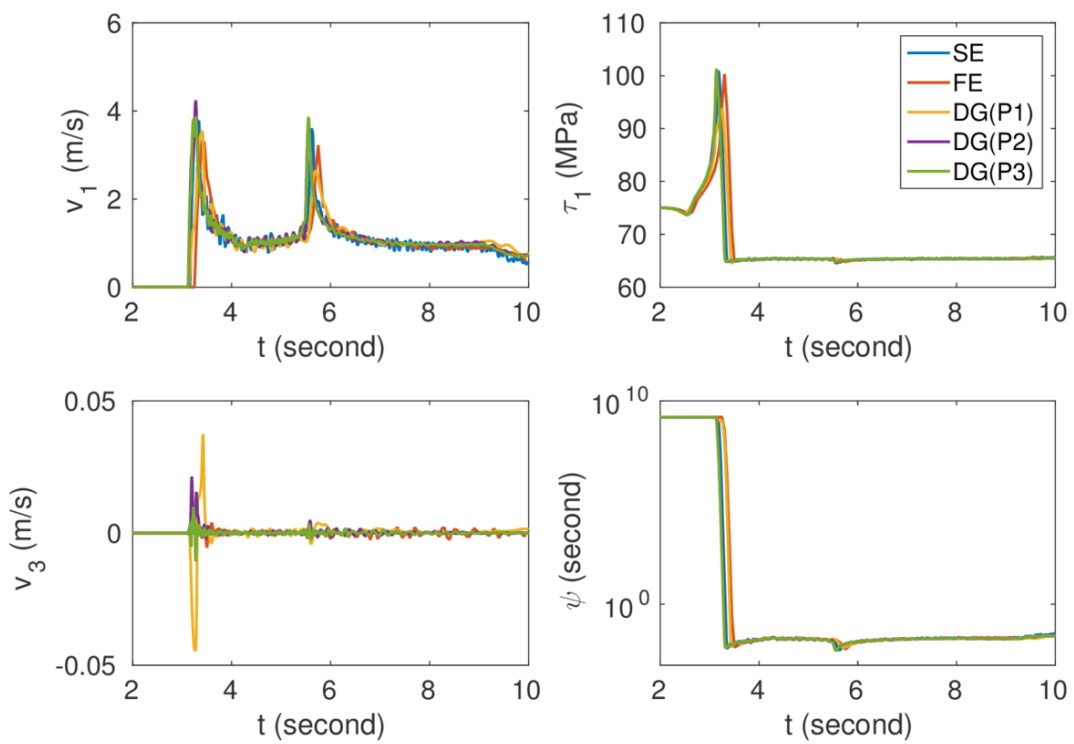

(b)
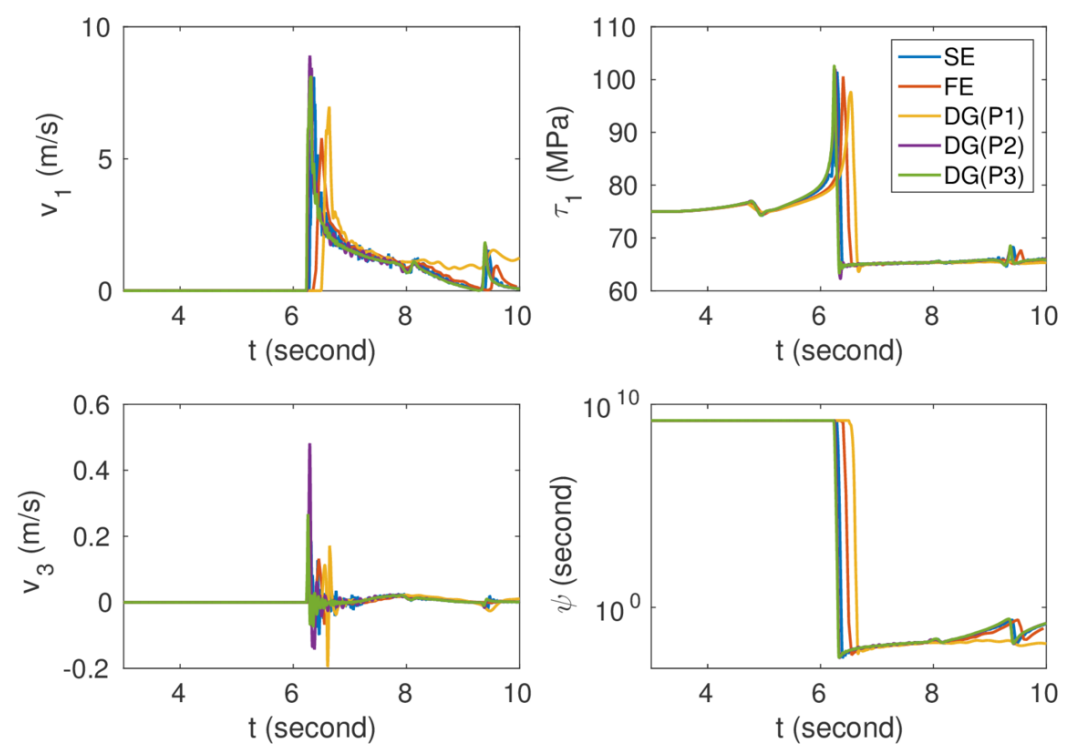

Fig. 5: Benchmark of the iterative coupling, DG method for polynomial orders 1,2 and 3, denoted respectively by "DG(P1)", "DG(P2)" and "DG(P3)" in the legend, against the spectral element (SE) method and the finite element (FE) method on "TPV102" with on-fault stations located at (a) $[0.0,-3.0,0.0] \mathrm{km}$, and (b) $[12.0,-12.0,0.0] \mathrm{km}$ (see also Figure 3 ), showing the horizontal slip rate $v_{1}$, horizontal shear stress $\tau_{1}$, vertical slip rate $v_{3}$ and state-variable $\psi$. 
(a)
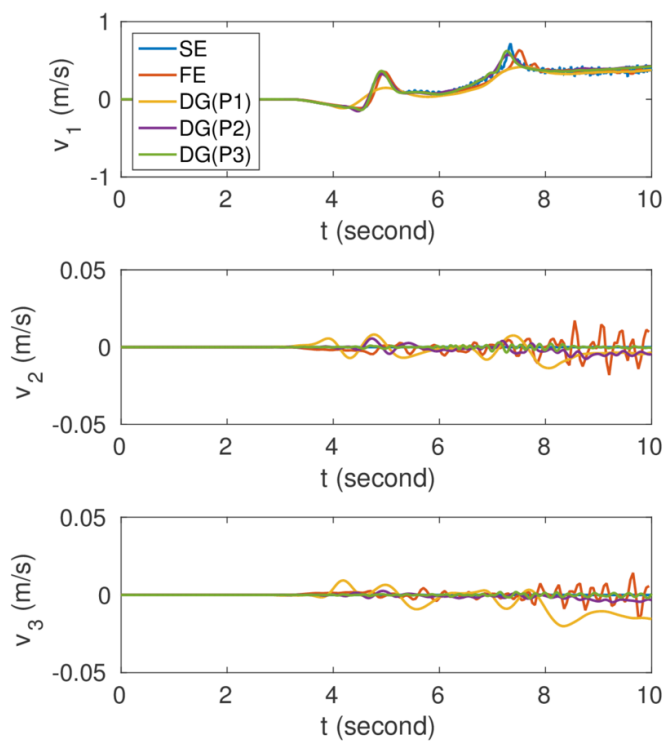

(b)
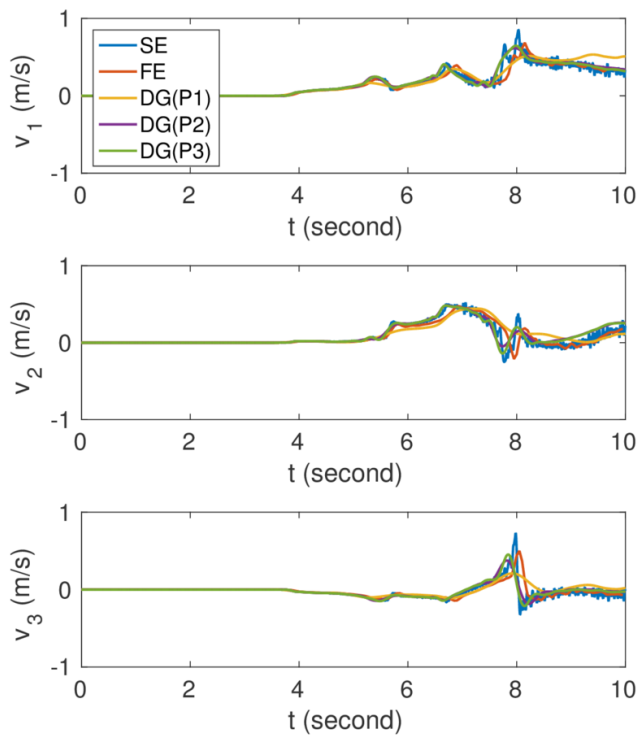

Fig. 6: Benchmark of the iterative coupling DG method for polynomial orders 1, 2, and 3, denoted respectively by "DG(P1)", "DG(P2)" and "DG(P3)" in the legend, with the spectral element (SE) method and the finite element (FE) method on TPV102 with on-ground stations located at (a) $[0.0,0.0,9.0] \mathrm{km}$ and (b) $[12.0,0.0,6.0] \mathrm{km}$, showing the horizontal velocity $v_{1}$, normal velocity $v_{2}$, and vertical velocity $v_{3}$.

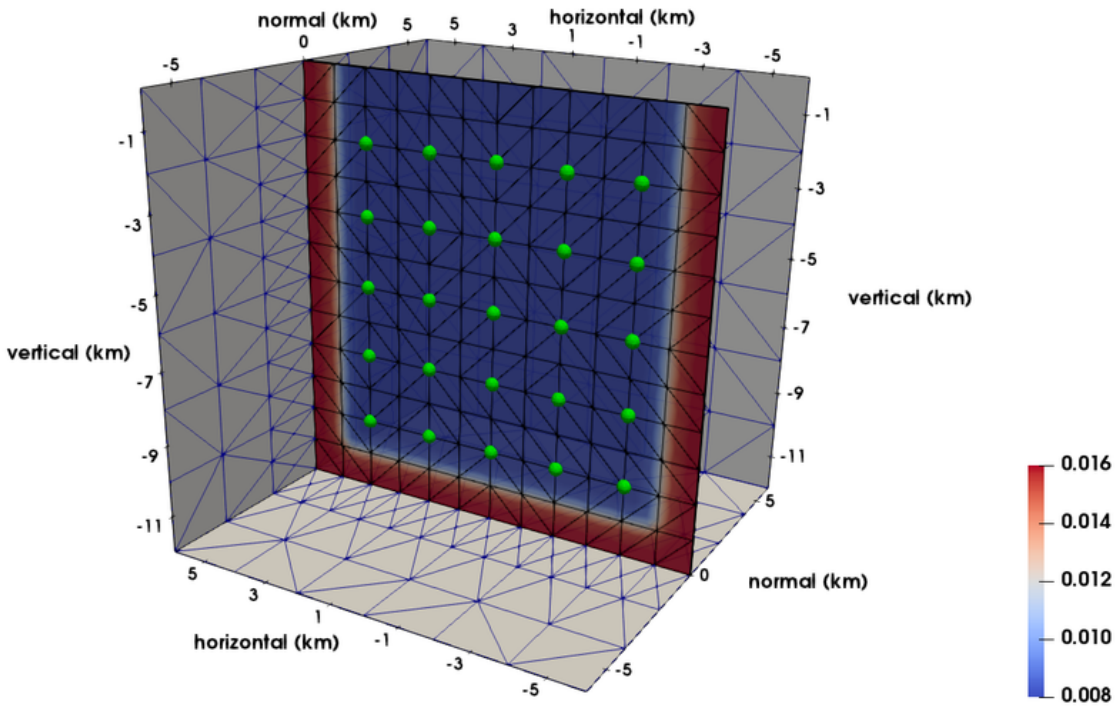

Fig. 7: The semi-regular tetrahedral mesh for one of the convergence test models. The color map shows the spatial distribution of parameter " $a$ " on the rupture plane, and the green dots represent the locations of 25 on-fault receivers based on which the numerical errors are computed. 
(a)

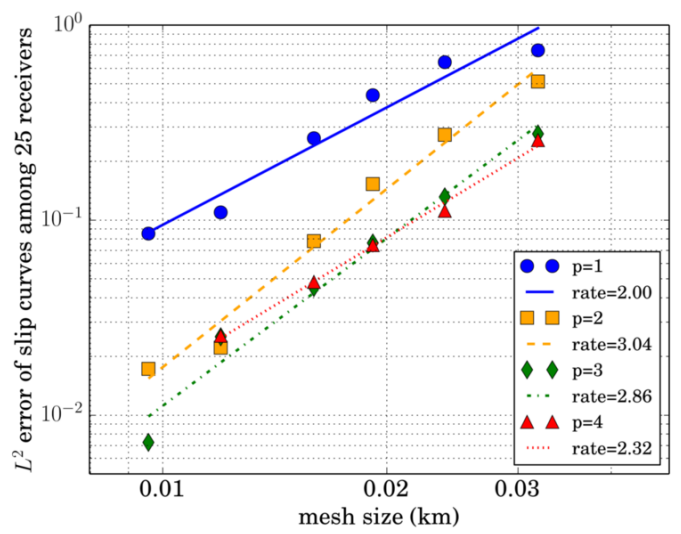

(b)

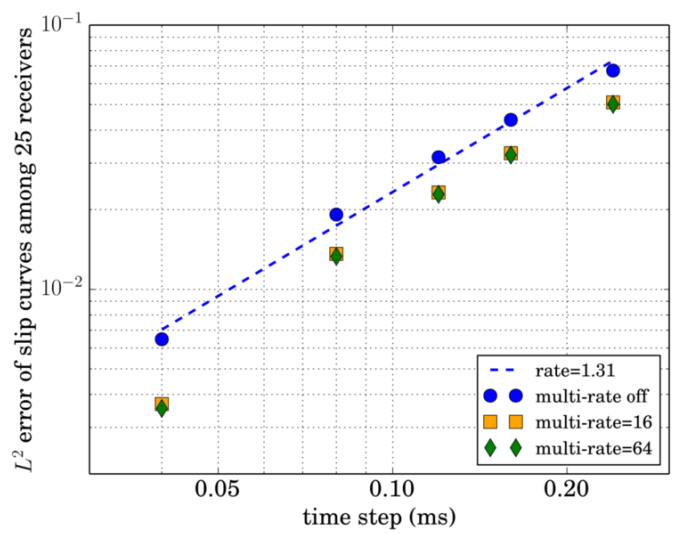

Fig. 8: (a) Numerical errors based on each mesh size $h$ and polynomial order $p$, relative to the numerical result with $h=9.57 \mathrm{~m}$ and $p=4$, with a linear regression fit showing the approximate convergence rate. (b) Numerical errors based on each time step $\Delta t$ and multi-rate substeps, relative to the numerical result with $\Delta t=0.02 \mathrm{~ms}$ and multi-rate $=64$, with a linear regression fit showing the approximate convergence rate.

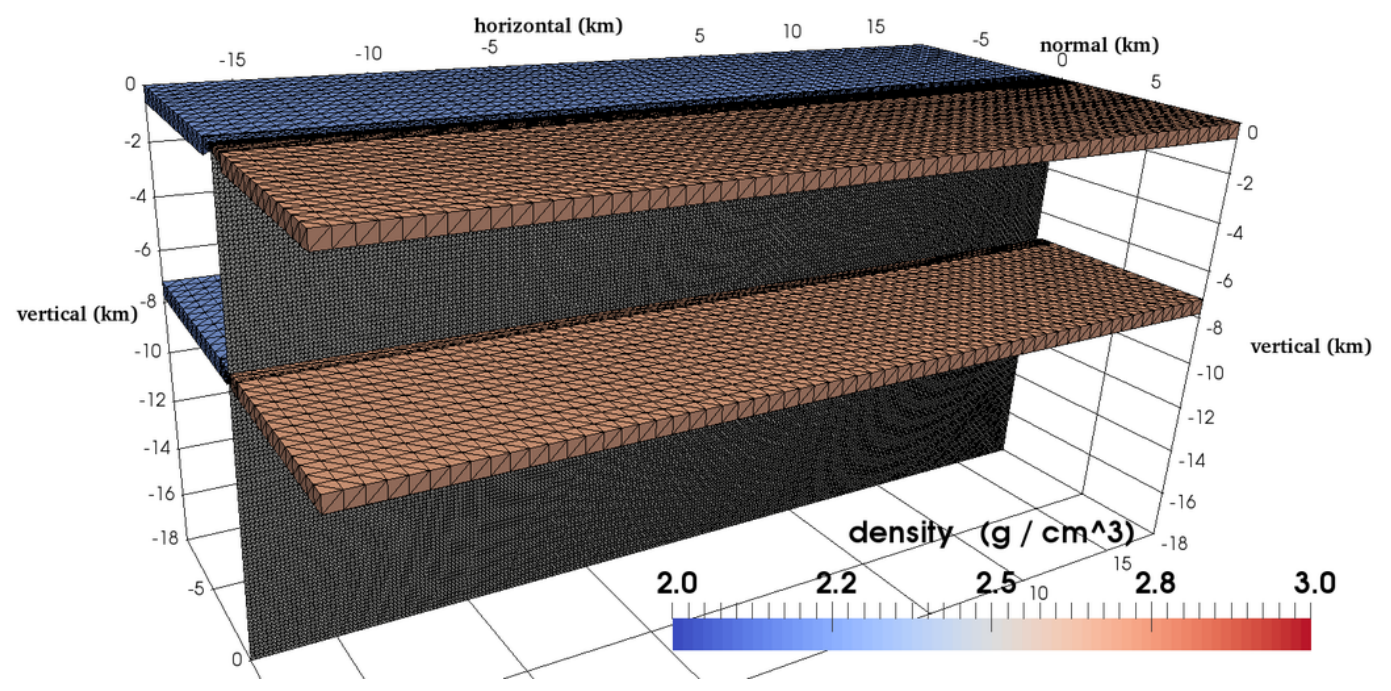

Fig. 9: Visualization of modified "TPV6" model in a quasi-regular tetrahedral mesh locally refined around the rupture with local mesh size $h=30 \mathrm{~m}$.

consisting of 2,101,840 elements, while the rupture surfaces are discretized with 52,340 triangles, with varying sizes based on the material parameters (see Figure 16). In the numerical simulation we use polynomial order 1 . We choose the viscosity coefficient elementwise, taking a constant value of $4.0 \times 10^{-7} \mathrm{GPa} \cdot \mathrm{s}$ in the elements attached to the rupture plane, and 0 in the remaining ones. We use a time step of $0.032 \mathrm{~ms}$ and a multi-rate scheme with 16 substeps. We show snapshots at $t=4.0 \sim 11.0 \mathrm{~s}$ of the simulation, with the 3 components of particle velocity in the volume listed in Figure 18-20. The propagation of rupture, and the time varying friction force, normal stress as 


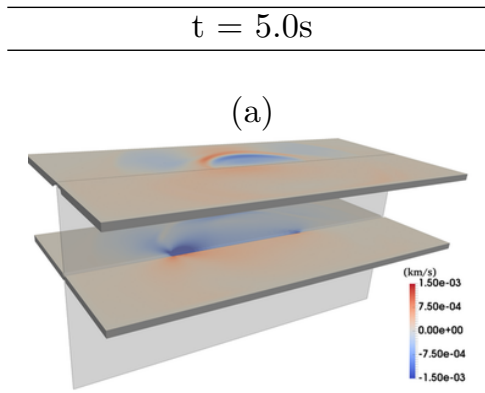

(d)

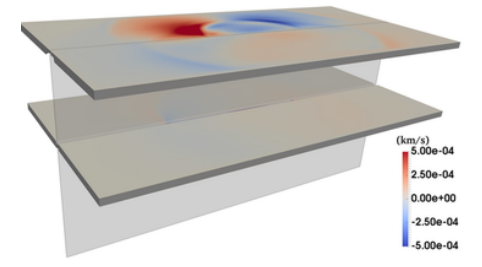

$(\mathrm{g})$

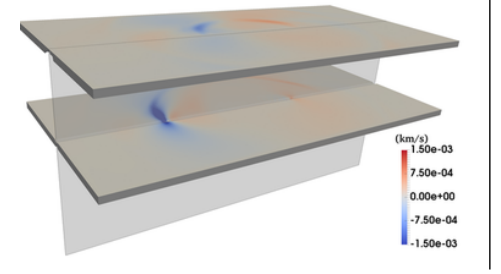

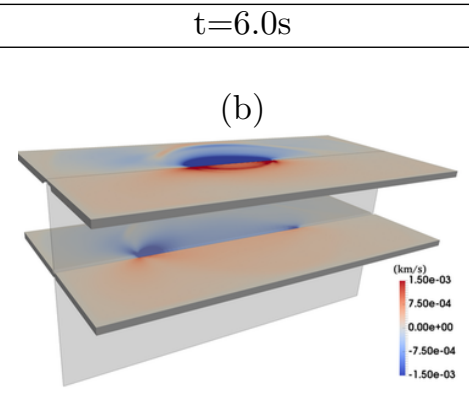

(e)

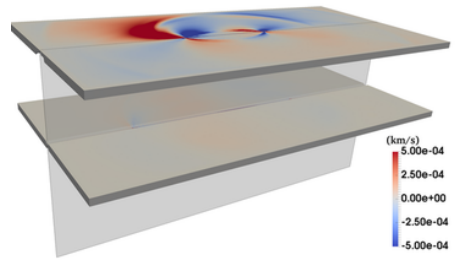

(h)

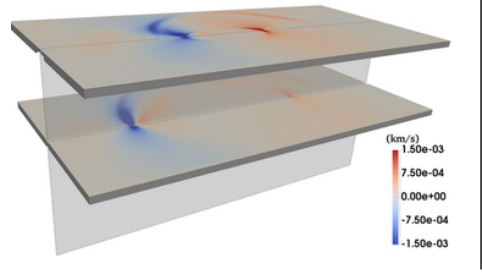

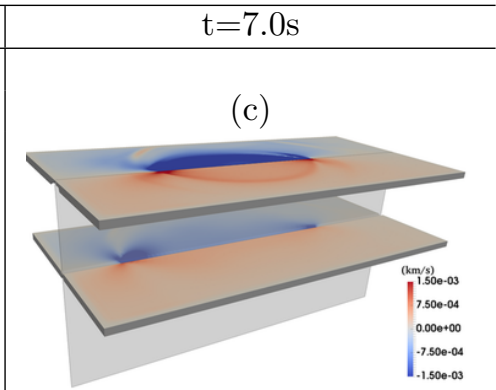

(f)

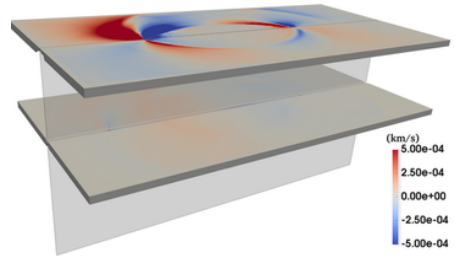

(i)

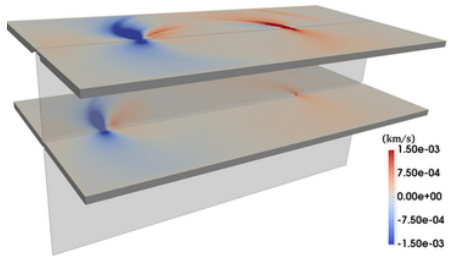

Fig. 10: Visualization of particle velocities in the modified "TPV6" model at $\mathrm{t}=5.0,6.0,7.0 \mathrm{~s}$ with (a, b, c) horizontal component, (d, e, f) vertical component, (g, h, i) normal component, computed by DG method with polynomial order 2 and $h=30 \mathrm{~m}$.

well as state variable are shown in Figures 17, 21 and 22. We also show seismograms at on-fault stations located at different depths, $-3.0,-7.5,-12.0 \mathrm{~km}$, in Figure 23. The numerical simulation reveals that the propagation of rupture is affected by scattering due to the geometry. In particular, the propagation speed reduces when it goes through a kink (Figure 17), which behavior is consistent with a theoretical analysis (Madariaga et al. [38]). The variation of compressive normal stress is significant around the corners of the rupture surface, as illustrated in Figure 22(a) and Figure 23(b). 


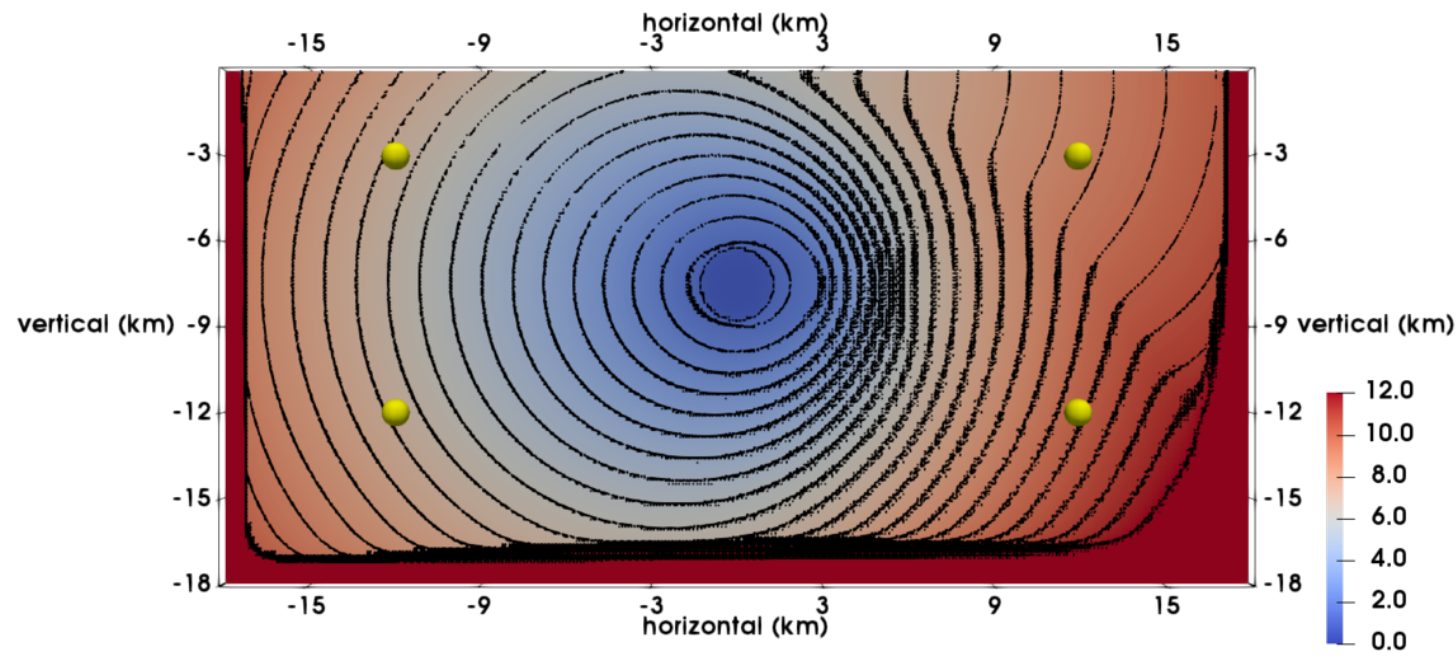

Fig. 11: Rupture contour plot of the modified "TPV6" model, with interval step of $0.5 \mathrm{~s}$. Balls in the figure are indicating the location of stations whose seismograms are plotted in Figure 13 and Figure 14.

\begin{tabular}{|c|c|c|c|}
\hline$V_{p}$ & $V_{s}$ & $\rho$ & $s_{c}$ \\
\hline $6.0 \mathrm{~km} / \mathrm{s}$ & $3.464 \mathrm{~km} / \mathrm{s}$ & $2.67 \mathrm{~g} / \mathrm{cm}^{3}$ & $10^{-6} \mu \mathrm{m} / \mathrm{s}$ \\
\hline \hline$a$ & $b$ & $L$ & $s_{0}$ \\
\hline $0.008 \sim 0.016$ & 0.012 & $2 \mathrm{~cm}$ & $1 \mu \mathrm{m} / \mathrm{s}$ \\
\hline \hline$f_{0}$ & $\left(\boldsymbol{T}_{0}\right)_{11}$ & $\left(\boldsymbol{T}_{0}\right)_{13}$ & $\left(\boldsymbol{T}_{0}\right)_{33}$ \\
\hline 0.6 & $-255 \mathrm{MPa}$ & $75 \mathrm{MPa}$ & $-120 \mathrm{MPa}$ \\
\hline
\end{tabular}

Table 5.3: Material parameters, rupture coefficients and prestress in the homogeneous-elastic stepping-over rupture model. The components of $\boldsymbol{T}_{0}$ not listed take the value 0 . The quantity $s_{c}$ is an aseismic (creeping) velocity that keeps $s$ away from 0. 


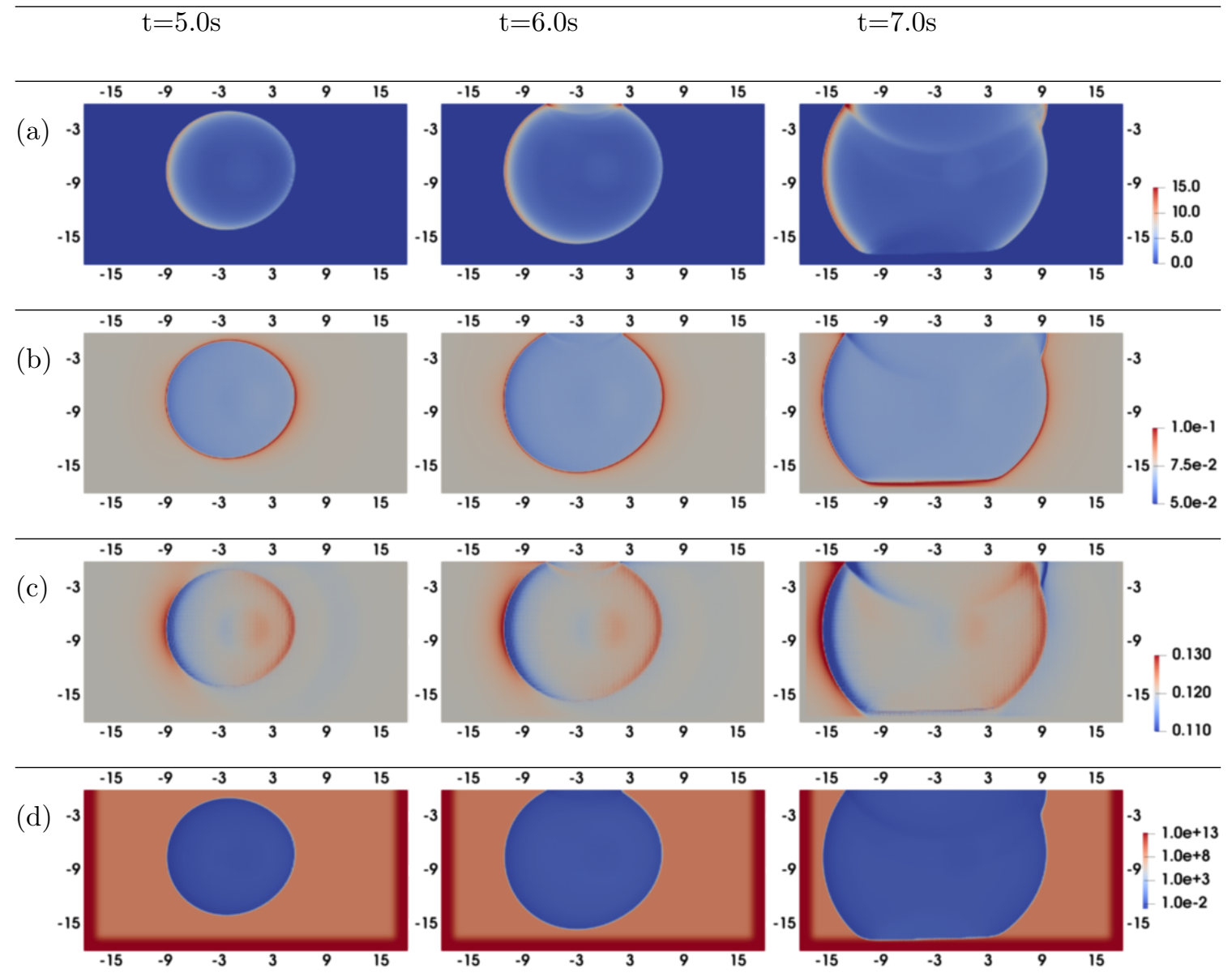

Fig. 12: Visualization on the rupture plane of modified "TPV6" model with (a) the slip rate with unit of $\mathrm{m} / \mathrm{s}$, (b) the magnitude of friction force with unit of GPa, (c) the compressive normal stress with unit of GPa, (d) the state variable ("age" of rupture) with unit of second, at time $\mathrm{t}=5.0,6.0$, $8.0 \mathrm{~s}$, computed by DG method with polynomial order 2 and $h=30 \mathrm{~m}$. 
(a)
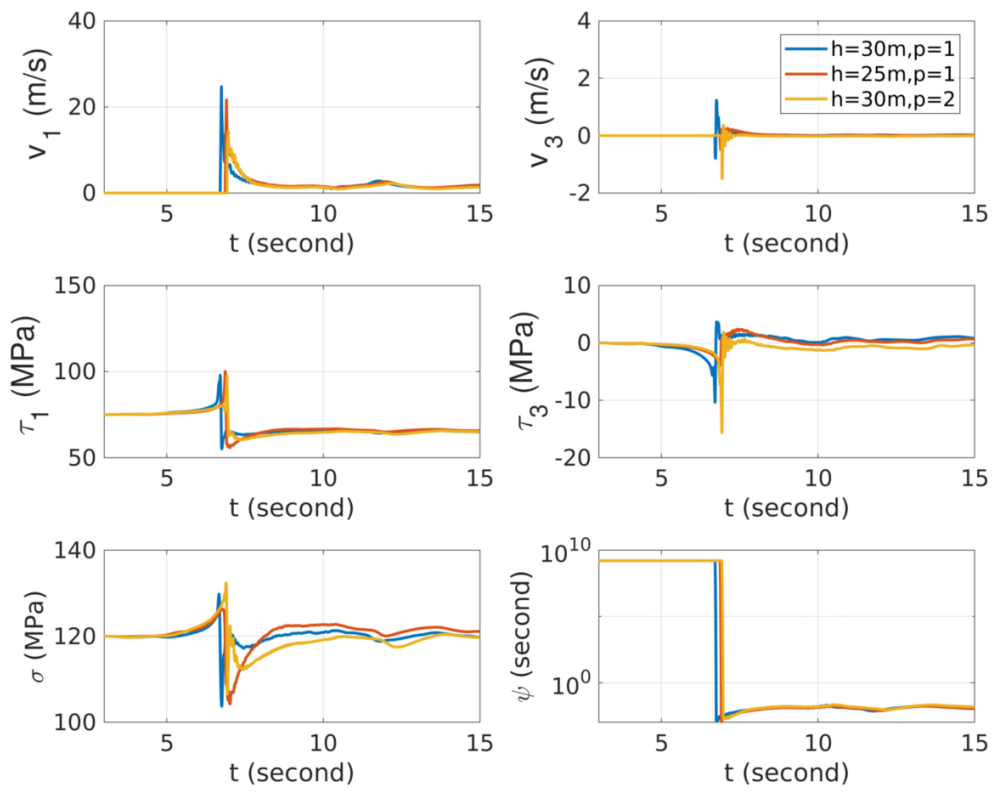

(b)
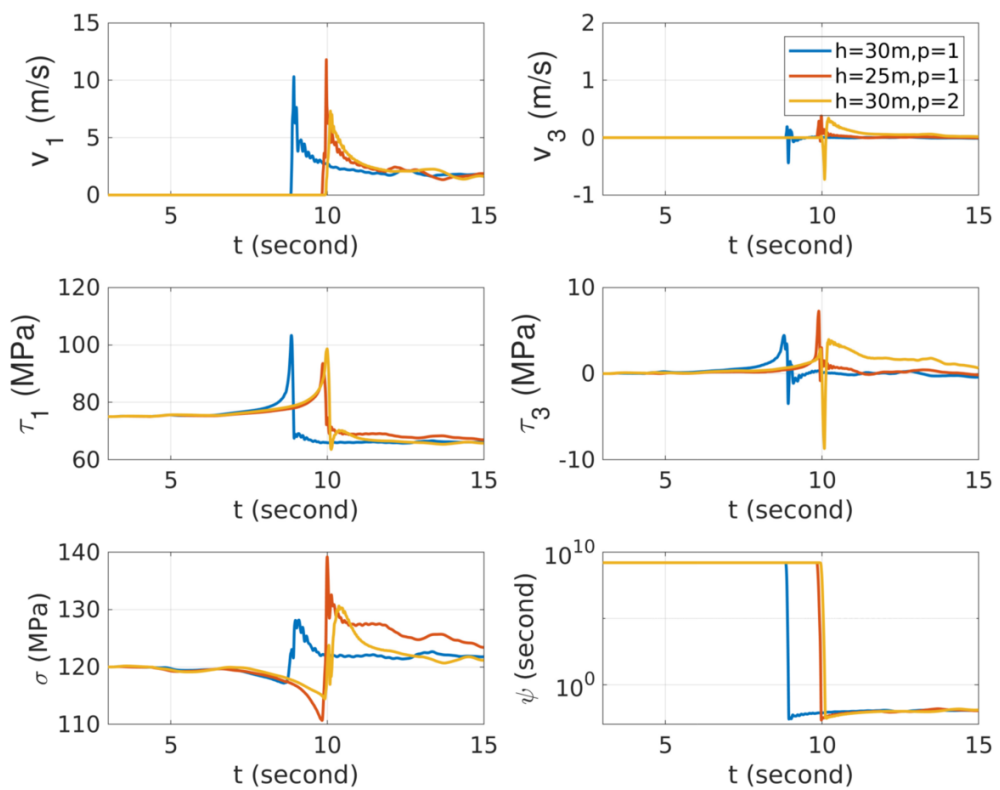

Fig. 13: Comparison of seismograms at on-fault stations located at (a) $[-12.0,-12.0,0.0] \mathrm{km}$, and (b) $[12.0,-12.0,0.0] \mathrm{km}$ of the modified "TPV6" model (see also Figure 11) with variant mesh size and polynomial order, showing the horizontal and vertical slip rate $v_{1}$ and $v_{3}$, horizontal and vertical shear stress $\tau_{1}$ and $\tau_{3}$, compressive normal stress $\sigma$ and state-variable $\psi$. 
(a)
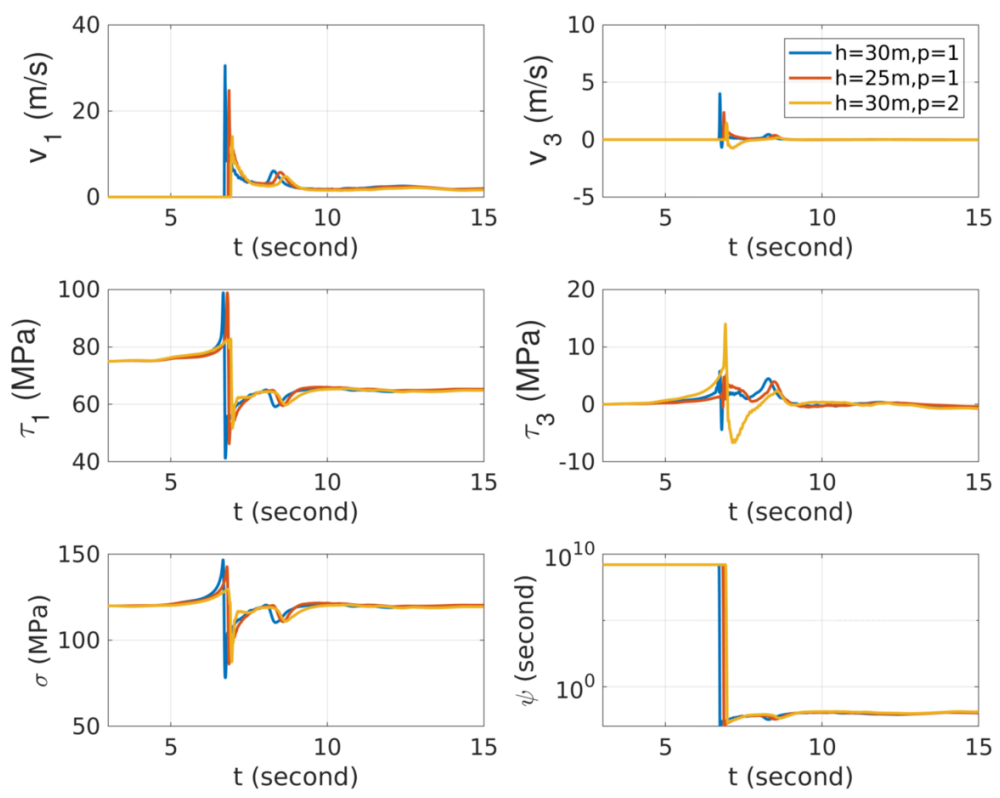

(b)
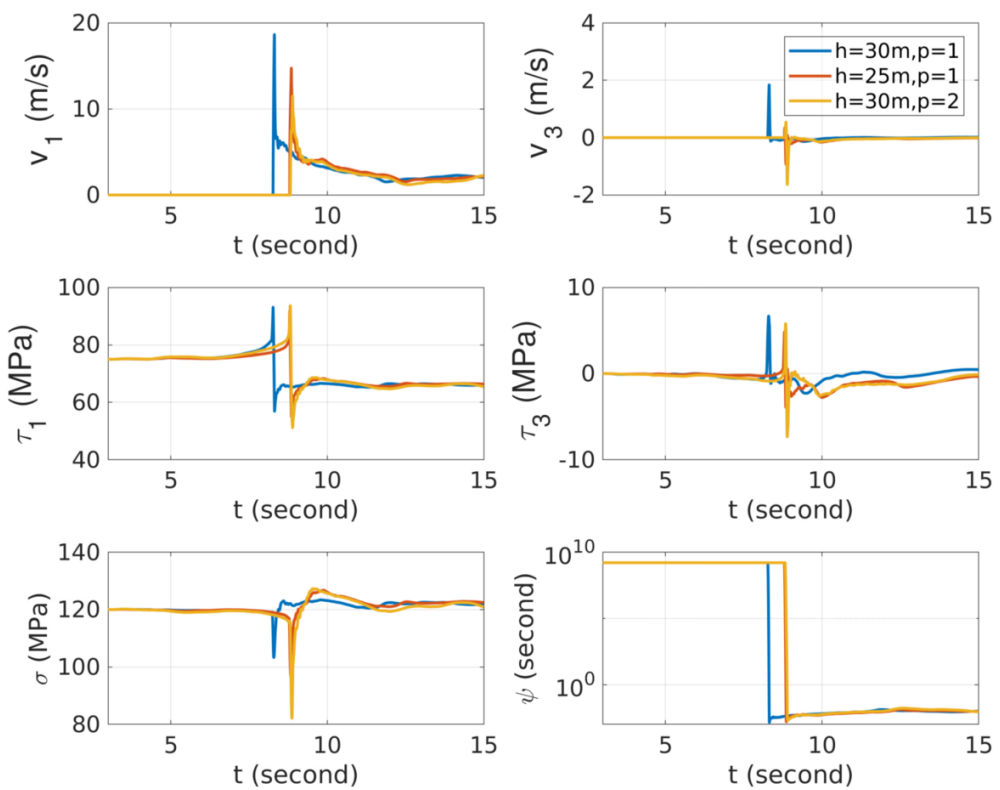

Fig. 14: Comparison of seismograms at on-fault stations located at (a) $[-12.0,-3.0,0.0] \mathrm{km}$, and (b) $[12.0,-3.0,0.0] \mathrm{km}$ of the modified "TPV6" model (see also Figure 11) with variant mesh size and polynomial order, showing the horizontal and vertical slip rate $v_{1}$ and $v_{3}$, horizontal and vertical shear stress $\tau_{1}$ and $\tau_{3}$, compressive normal stress $\sigma$ and state-variable $\psi$. 
(a)
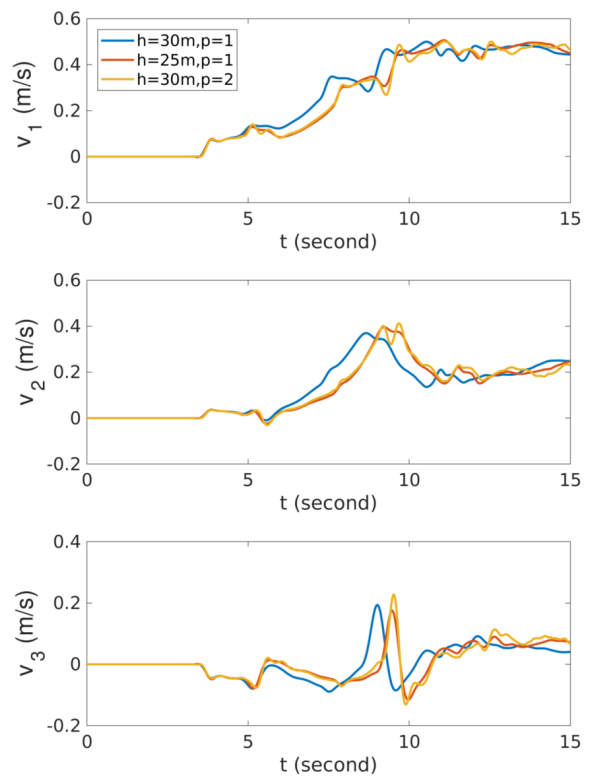

(b)
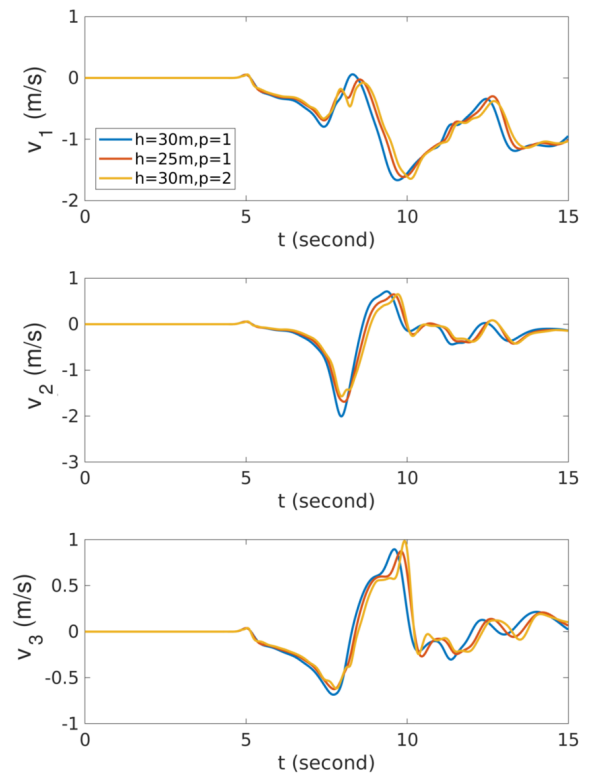

Fig. 15: Comparison of seismograms at on-ground stations located at (a) $[12.0,0.0,6.0] \mathrm{km}$, and (b) $[-12.0,0.0,-6.0] \mathrm{km}$ of the modified "TPV6" model with variant mesh size and polynomial order, showing the horizontal velocity $v_{1}$, normal velocity $v_{2}$, and vertical velocity $v_{3}$.

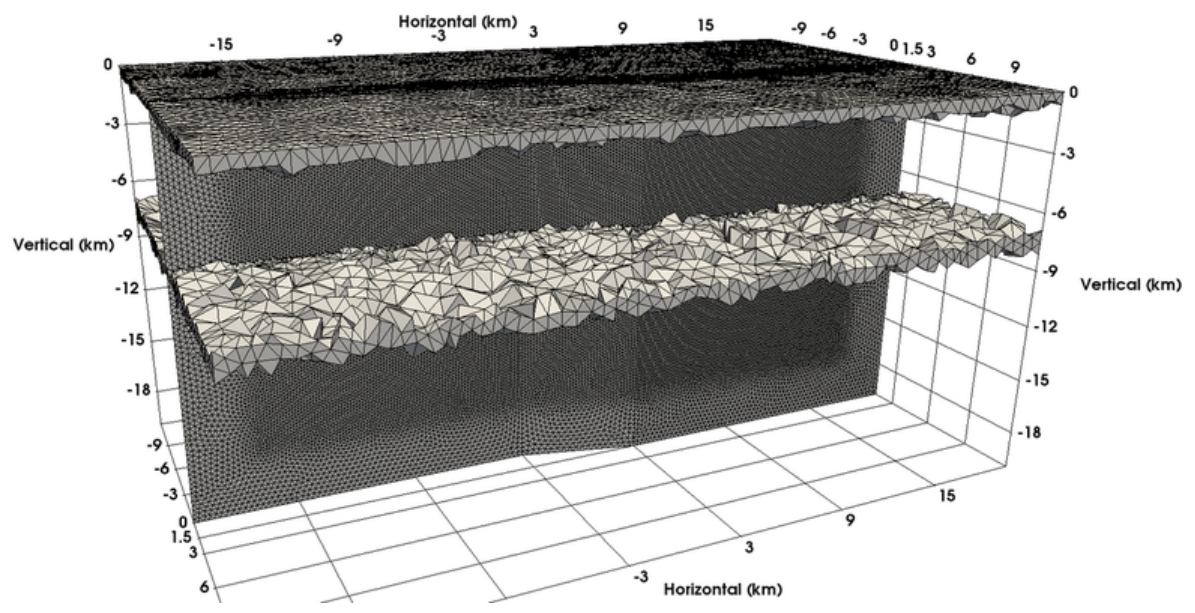

Fig. 16: Visualization of stepping-over fault model with unstructured tetrahedral mesh. 


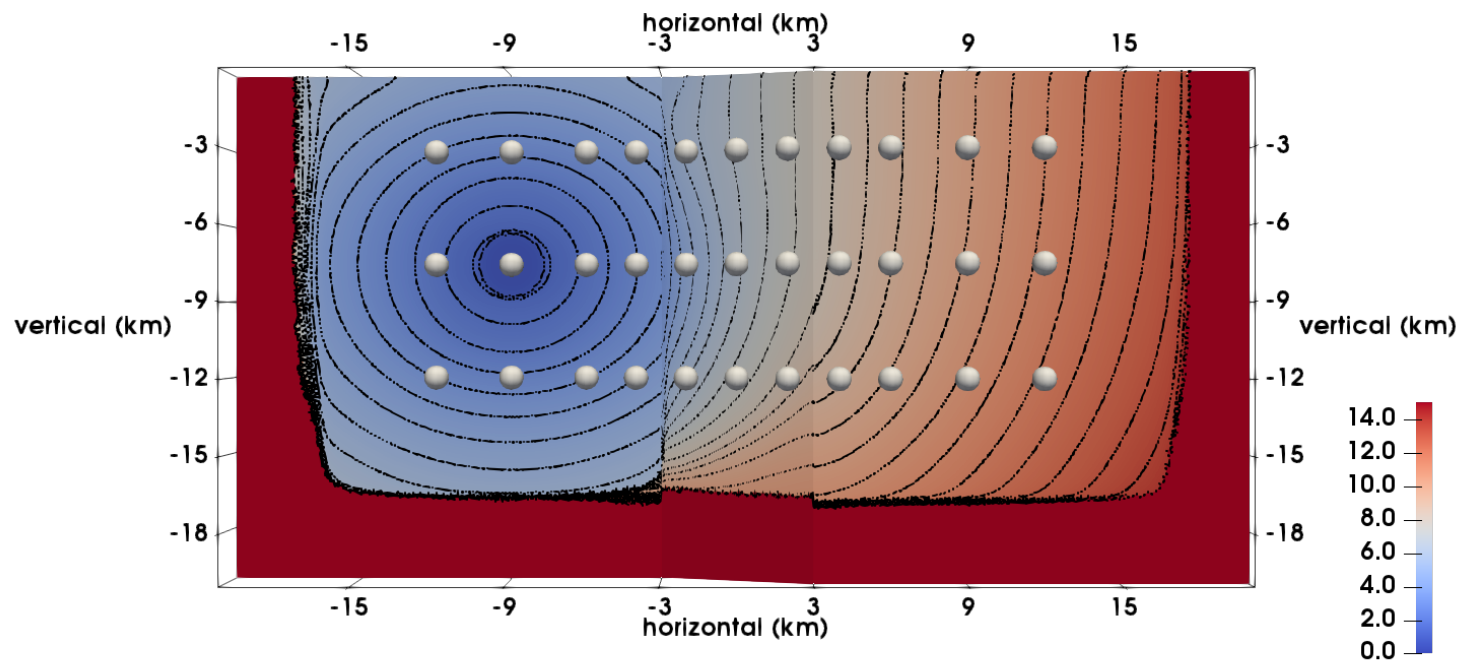

Fig. 17: Rupture contour plot of the stepping-over fault model, with interval step of $0.5 \mathrm{~s}$. Balls in the figure are indicating the location of stations whose seismograms are plotted in Figure 23. 

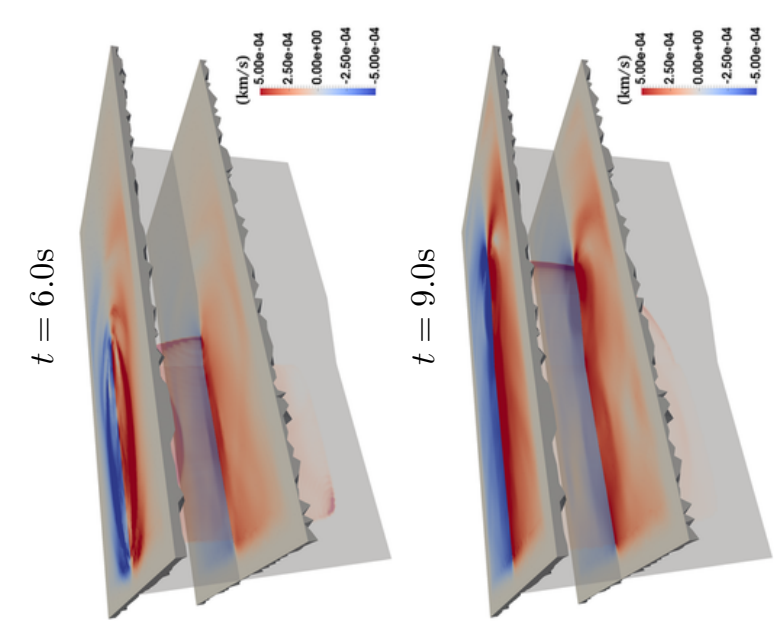

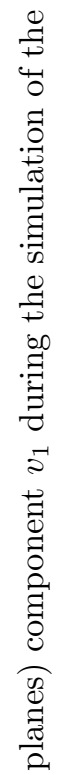
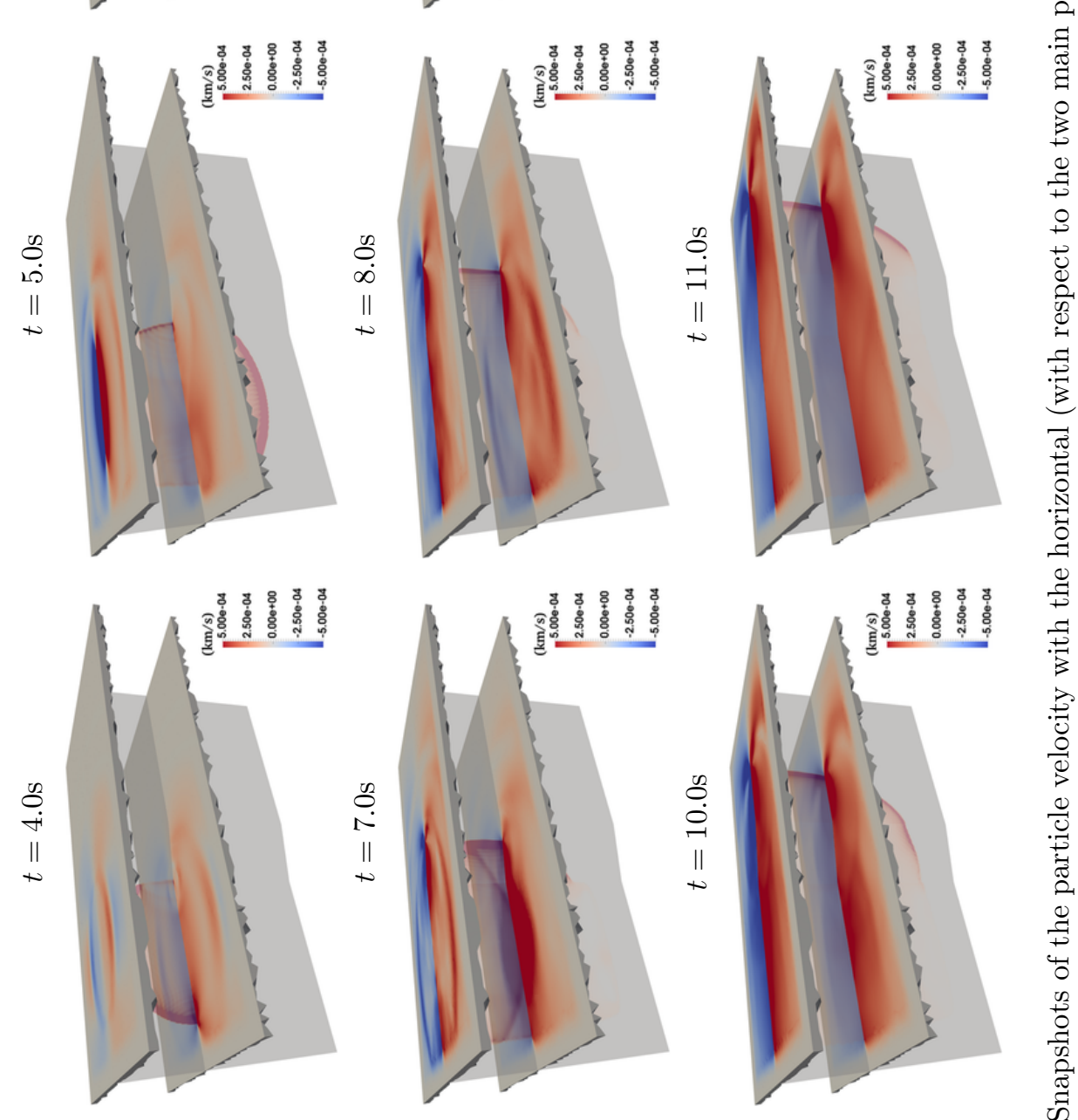

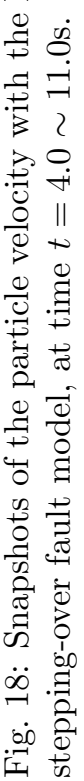



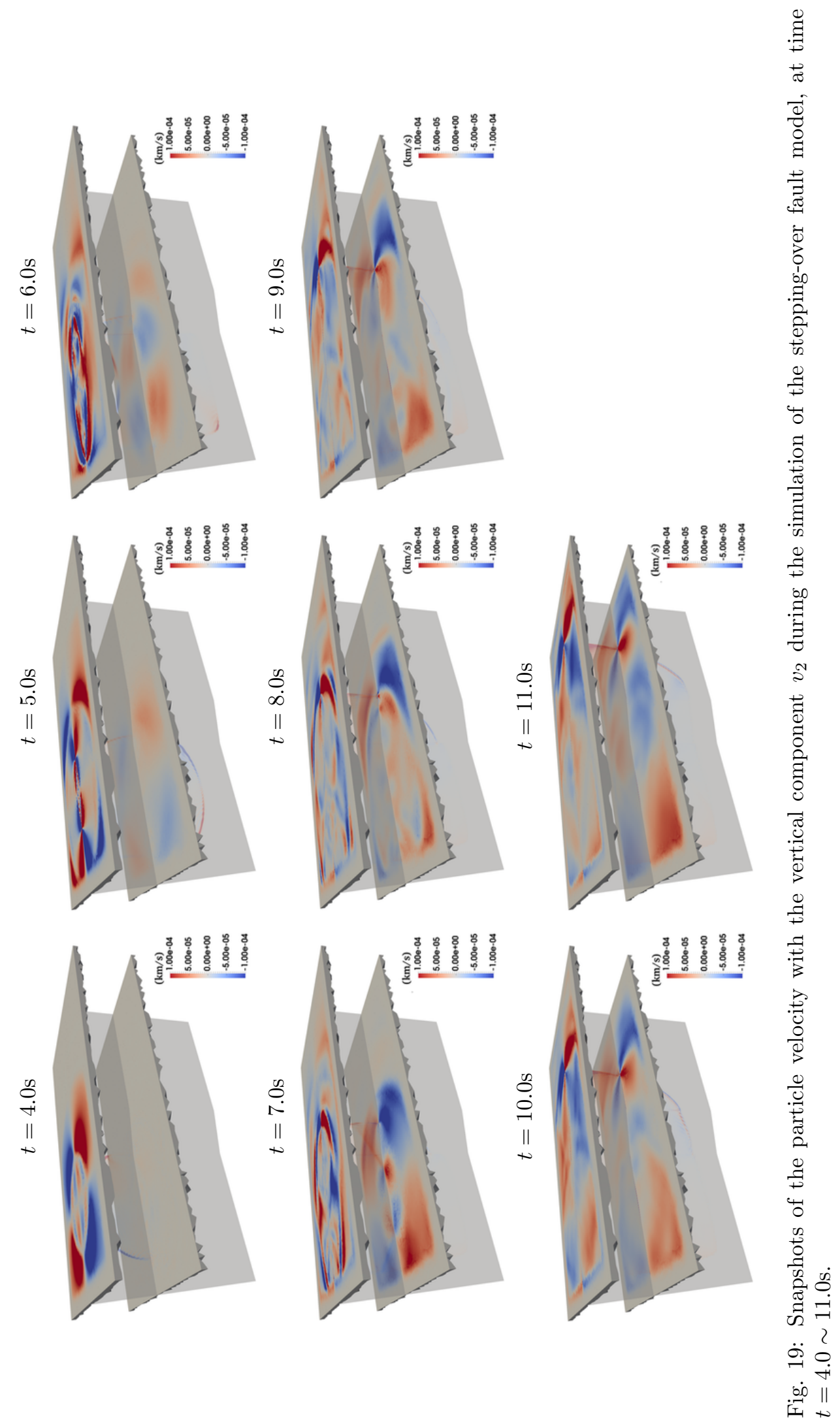

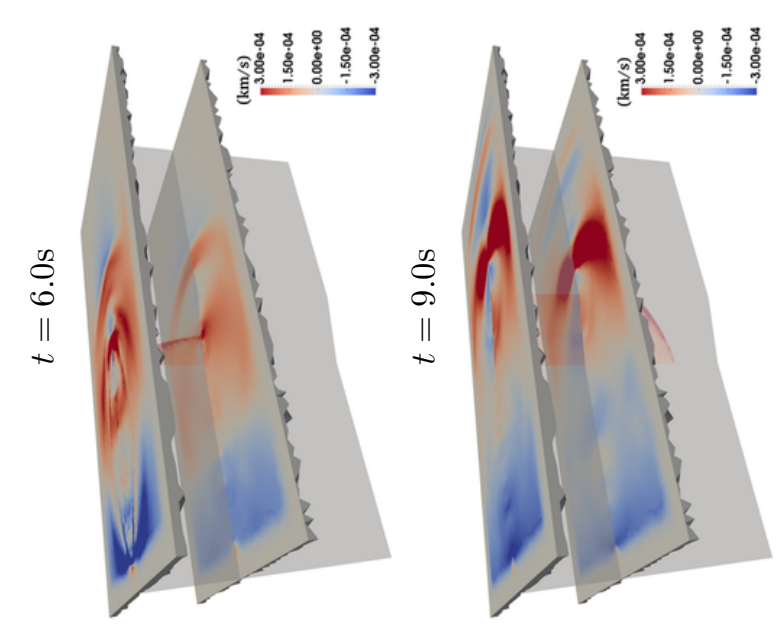

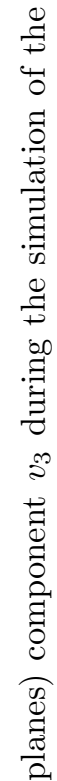
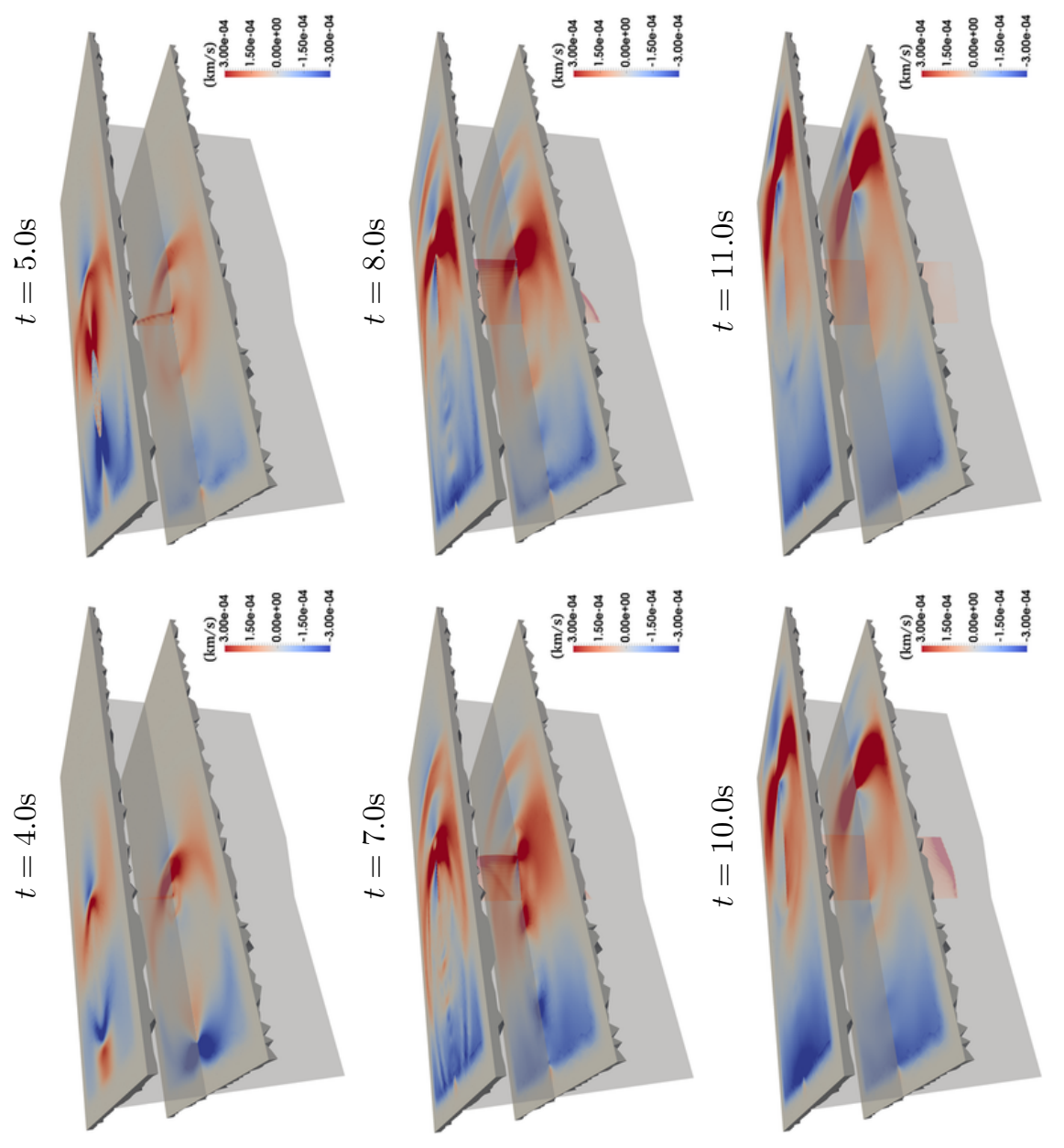

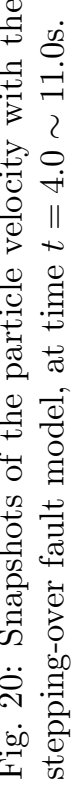




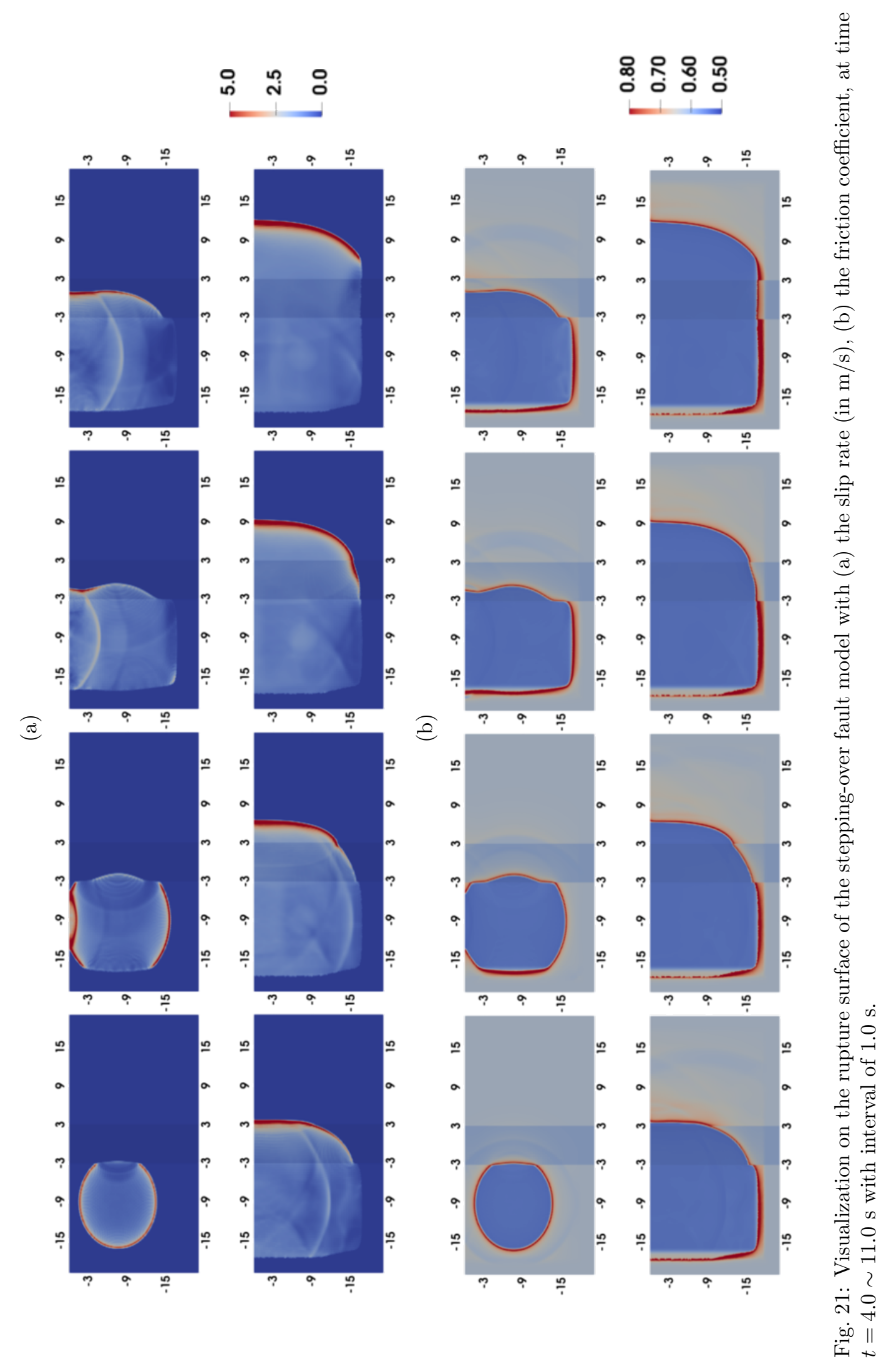




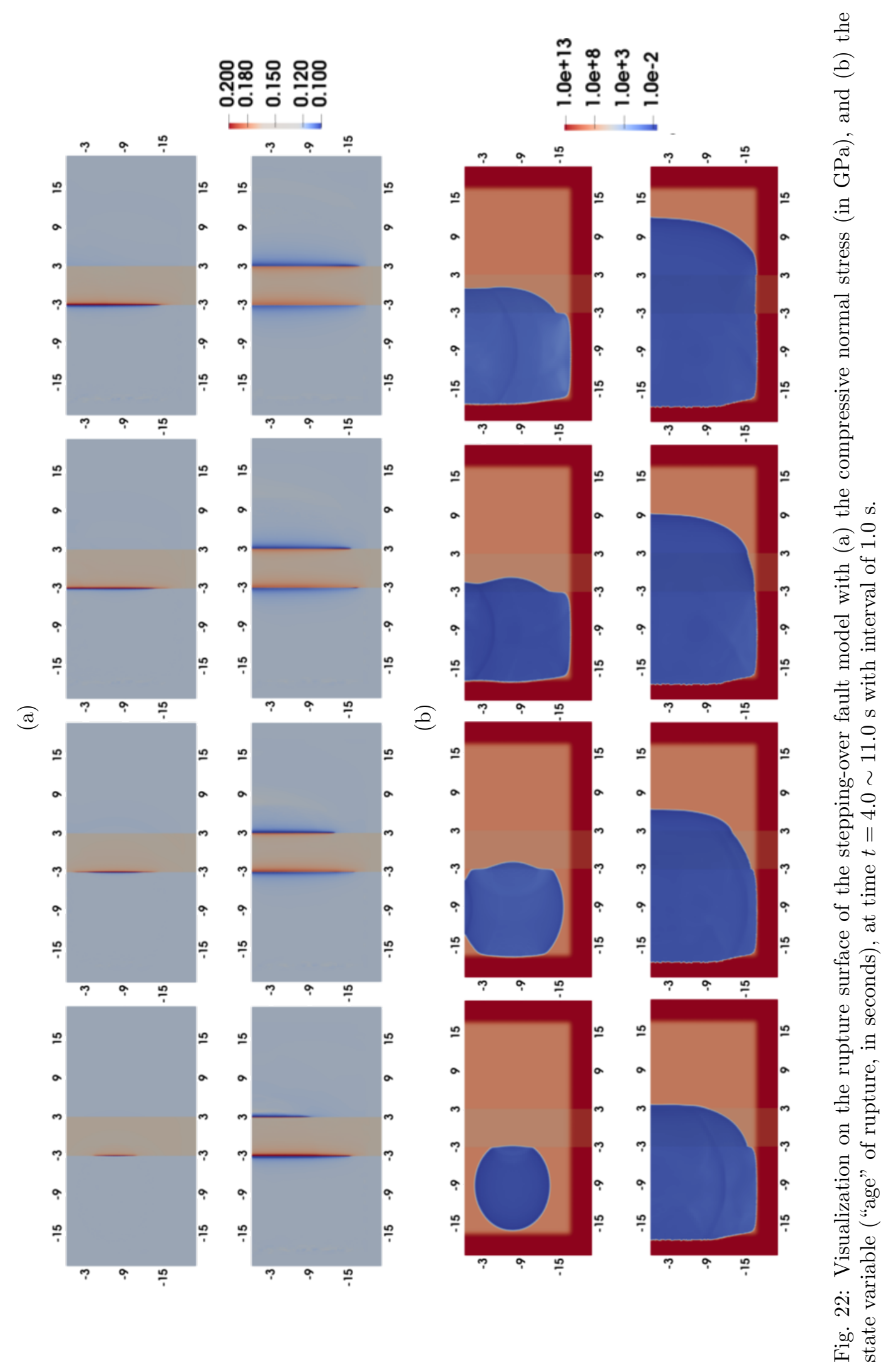


5.5. The impact of artificial viscosity on rupture propagation. In Theorem A.1 (A) and Theorem B.1 (B), we give lower bounds for the viscosity coefficient guaranteeing stability. The general impact of viscosity on the evolution of rupture dynamics are outside the scope of this paper. Nevertheless, we show an example demonstrating the importance of choosing an appropriate value of viscosity coefficient that is sufficiently large for stability, but small enough to preserve the physical properties of the original problem.

We consider the non-planar rupture problem described in Section 5.4, and choose a set of relative; large viscosity coefficients, $2.0 \times 10^{-5}, 4.0 \times 10^{-5}$ and $4.0 \times 1.0^{-4} \mathrm{GPa} \cdot \mathrm{s}$, within the elements attached to the rupture surface, and 0 in the rest ones. The time step used in all four tests is $0.032 \mathrm{~ms}$.

We show snapshots of the slip rate at $t=6.0 \mathrm{~s}$, when the rupture propagates across the first corner, for different values of viscosity coefficient in Figure 24. The comparison of rupture contour is shown in Figure 25. As a general observation from the numerical simulations, the propagation speed of rupture decreases with increasing viscosity. The impact of viscosity can be significant for a rupture surface with non-planar geometry, and result in a distinct propagation pattern. With increasing viscosity the propagation speed of slip is reduced (the left column of Figure 24), while the variation of normal stress coincides with the slip propagation along the kinks (the right column of Figure 24). Energy analysis [46, Section 2.1.1] provides hints for the impact of viscosity on the interaction between normal stress perturbation and rupture propagation.

5.6. The impact of static self-gravitation and prestress on rupture propagation. Here, we show the impact of static self-gravitation and prestress on the rupture propagation in the planar fault example "TPV102". We consider a plane fault residing in the crust of a spherical reference model (PREM), see [23]). The top of the rupture plane intersect with the surface of earth. We assume that the initial gravitational potential is generated by the reference density of PREM, and the prestress is given in Table 5.1. We employ the Cowling approximation, which ignores the perturbation of gravitational potential. Otherwise, we use the model, and mesh with $p=2$, described in Section 5.1.

In Figure 26, we show the difference of rupture contours obtained from the numerical simulations based on the non-prestressed assumption and the one that accounts for the initial gravitational potential and prestress. We also compare the seismograms of stations located on the fault plane in Figure 27. With the presence of prestress, the relation between the stress tensor and the particle displacement is altered at each fixed location, based on the Piola transform between Eulerian and Lagrangian coordinates, which contributes the most to the difference in propagation speed of rupture on a planar fault. The strike-slip rupture has nearly horizontal particle motion, and the impact of the static gravitity field to the dynamic process is minor. The normal stress is hardly impacted as well which is partly due to orientation of the fault plane. The dynamic effects of self-gravitation upon nucleation are not included in this example, and will be considered in future work.

6. Discussion. We introduce a novel multi-rate iterative scheme coupling the elastic wave equation with rupture dynamics based on rate- and state-dependent friction laws on faults. We use discontinuous Galerkin method, with a modified penalty flux, in which the friction law is integrated in the weak form of particle motion as numerical flux. The time scale for rupture dynamics is significantly different from that for elastic wave propagation and scattering. In our splitting approach, we take several finer implicit time steps for the state ODE within each coarse time step for wave propagation and scattering. As the analysis shows, this splitting is a contraction in natural norms. We have tested our numerical approach and algorithm on several spontaneous rupture problems. We have also shown convergence results with polynomial refinement, and benchmarks against commonly used algorithms.

Acknowledgements. R. Ye acknowledges the support from the Simons Foundation under the MATH + X program, and from the members of the Geo-Mathematical Imaging Group at Rice University. K. Kumar acknowledges Toppforsk, Norwegian Research Council project 250223. M. V. de Hoop acknowledges the support from the Simons Foundation under the MATH + X program, 
(a)
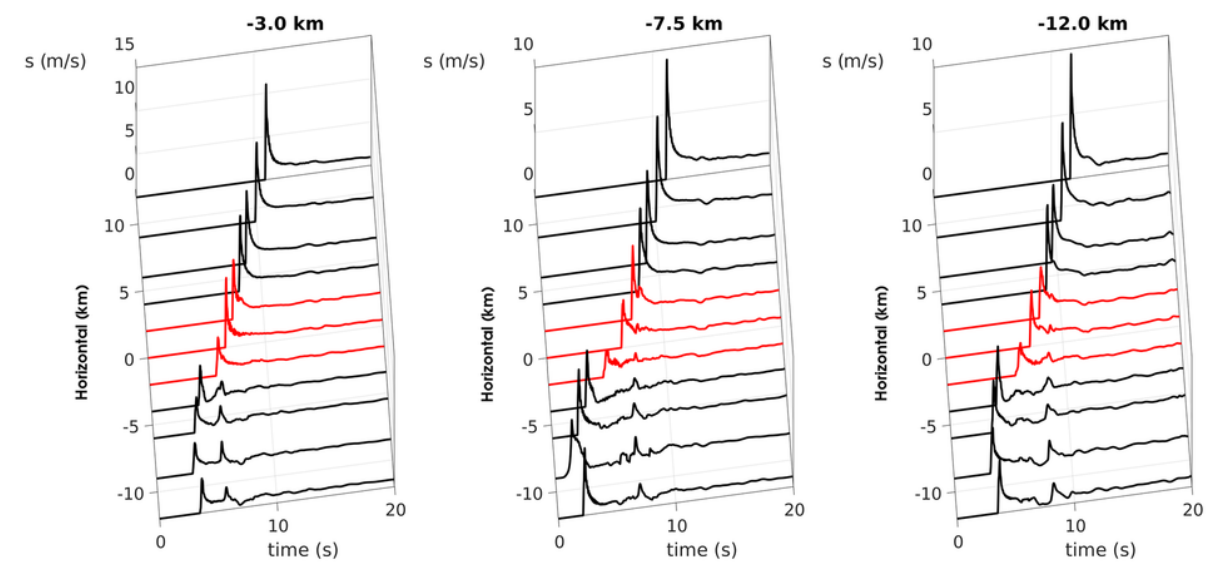

(b)
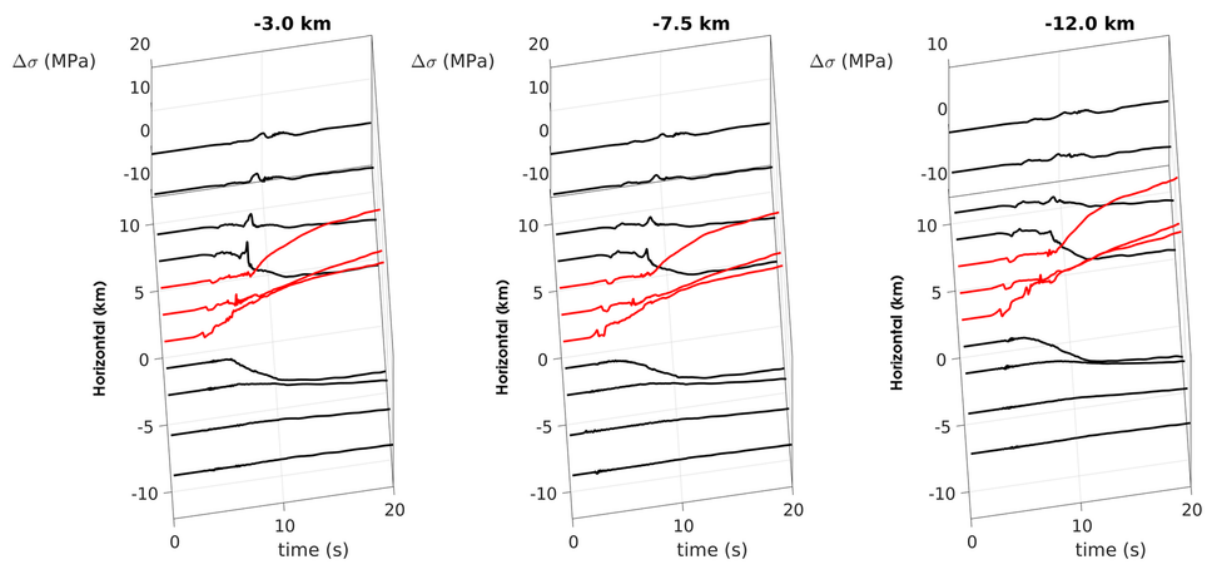

(c)
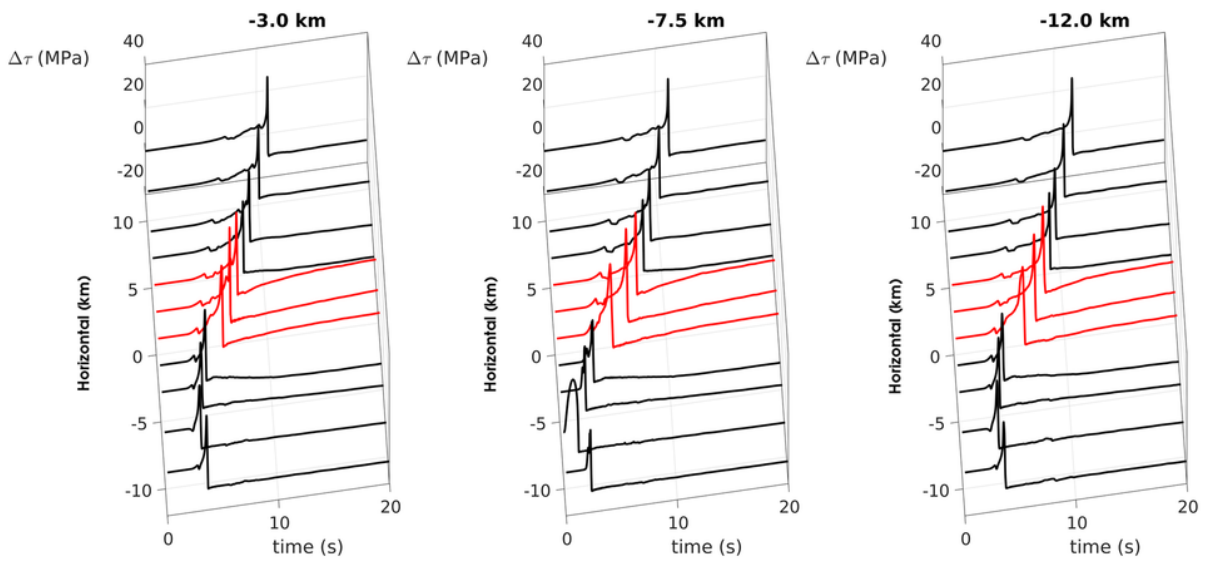

Fig. 23: Seismograms at on-fault stations located at horizontal location $x_{1}=-12.0 \sim 12.0 \mathrm{~km}$ and depth $x_{2}=-3.0,-7.5,-12.0 \mathrm{~km}$ of the modified step-over model (see also Figure 17), showing (a) the amplitude of slip rate $s$, (b) the increment of compressive normal stress $\Delta \sigma=\sigma+\left(\boldsymbol{n} \cdot T^{0} \cdot \boldsymbol{n}\right)$, and (c) the incremental amplitude of shear stress $\Delta \tau=\left|\boldsymbol{\tau}_{\mathrm{f}}-\left(\boldsymbol{n} \cdot \boldsymbol{T}^{0}\right)_{\|}\right|$. Red plots are seismograms on the intermediate plane between two kinks. 


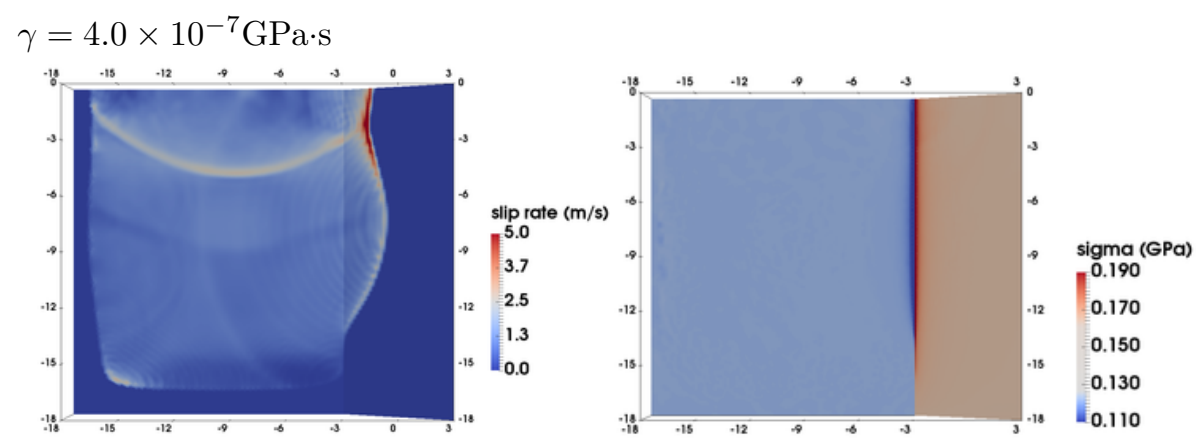

$\gamma=2.0 \times 10^{-5} \mathrm{GPa} \cdot \mathrm{s}$
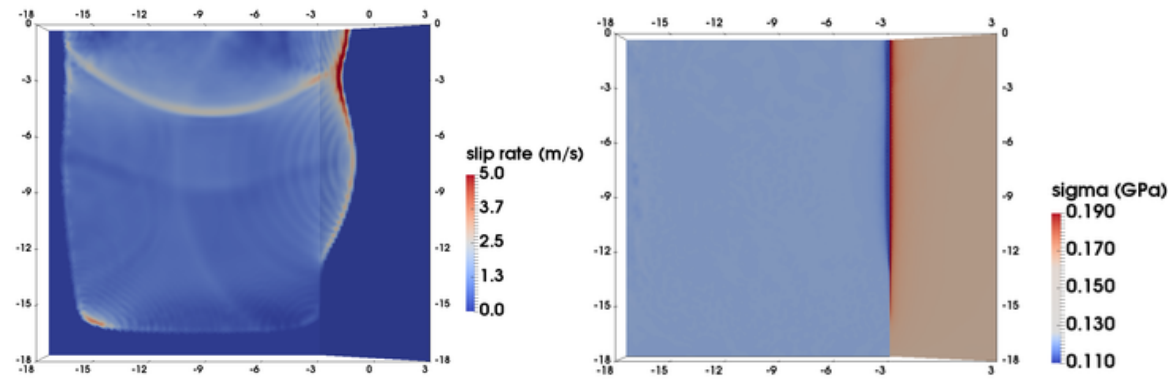

$\gamma=4.0 \times 10^{-5} \mathrm{GPa} \cdot \mathrm{s}$
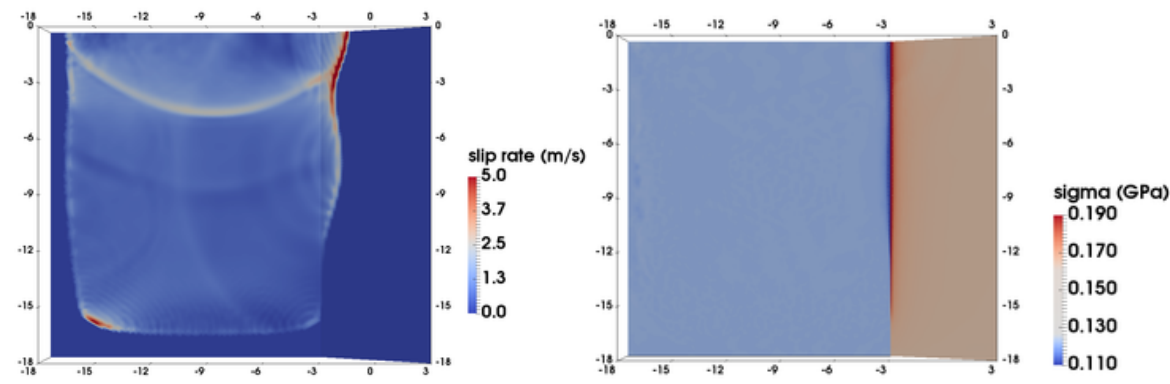

$\gamma=1.0 \times 10^{-4} \mathrm{GPa} \cdot \mathrm{s}$
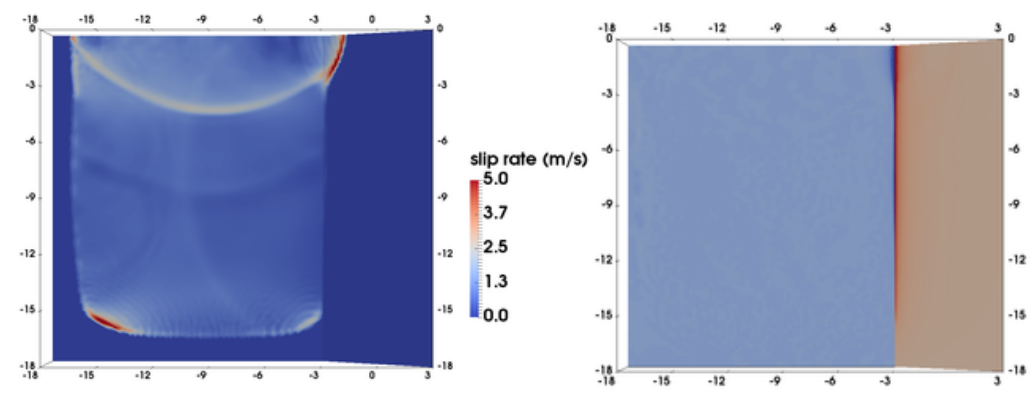

sigma (GP
0.190
0.170
0.150
0.130
0.110

Fig. 24: Visualization of slip rate (left column) and normal compressive stress (right column) at the rupture surface of the stepping-over fault model at time $t=6.0$ s with different viscosity coefficients. 


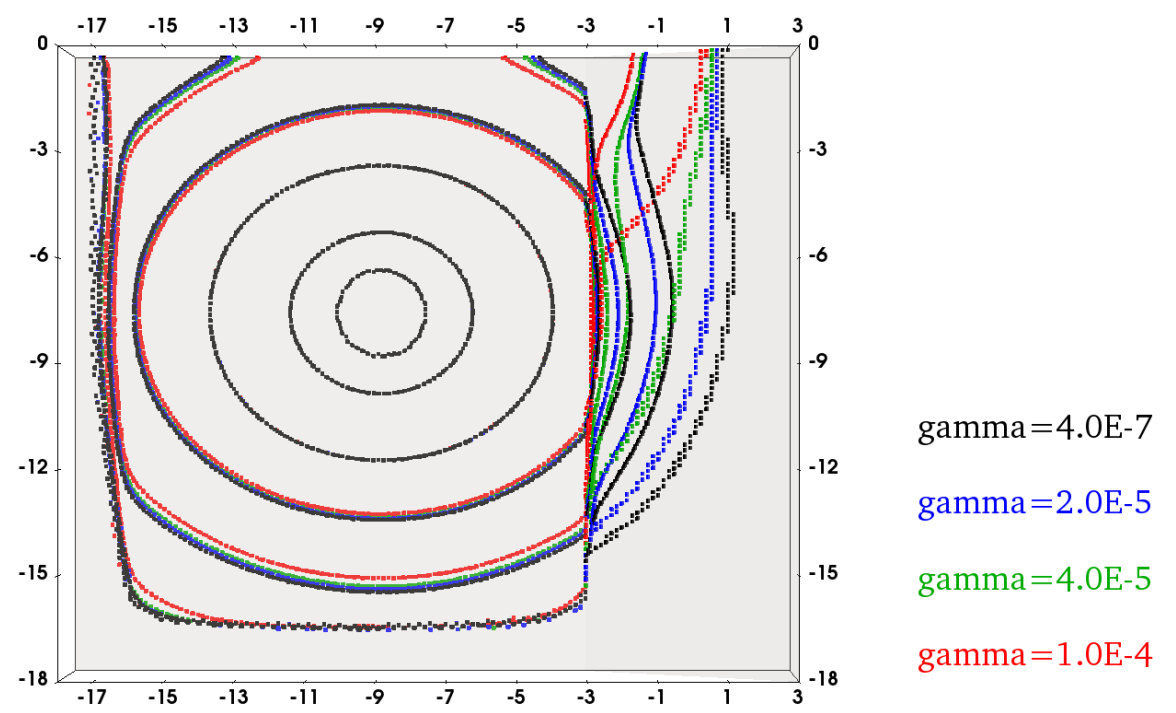

Fig. 25: Comparison of rupture contour of the stepping-over fault model with different values of the viscosity coefficient: $\gamma=4.0 \times 10^{-7} \mathrm{GPa} \cdot \mathrm{s}$ (black), $2.0 \times 10^{-5} \mathrm{GPa} \cdot \mathrm{s}$ (blue), $4.0 \times 10^{-5} \mathrm{GPa} \cdot \mathrm{s}$ (green), $1.0 \times 10^{-4} \mathrm{GPa} \cdot \mathrm{s}$ (red). Contours are plotted from 1.0 to $7.0 \mathrm{~s}$ with the interval of $1.0 \mathrm{~s}$.

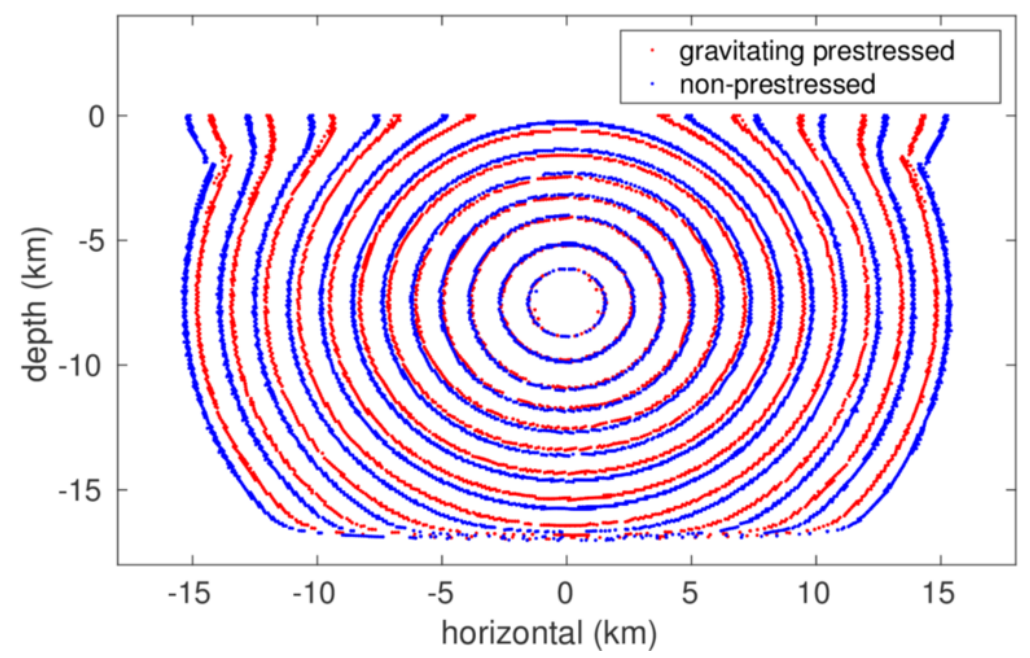

Fig. 26: Comparison of rupture contours of "TPV102" model by numerical simulations with/without consideration of the initial gravitational potential and prestress. Interval step is $0.5 \mathrm{~s}$.

from the National Science Foundation under grant DMS-1559587, and from the members of the GeoMathematical Group at Rice University. M. Campillo acknowledges the support from the European Research Council (ERC) under the European Unions Horizon 2020 research and innovation program (grant agreement No 742335, F-IMAGE).

Appendix A. A priori estimate for solutions of the coupled system. 

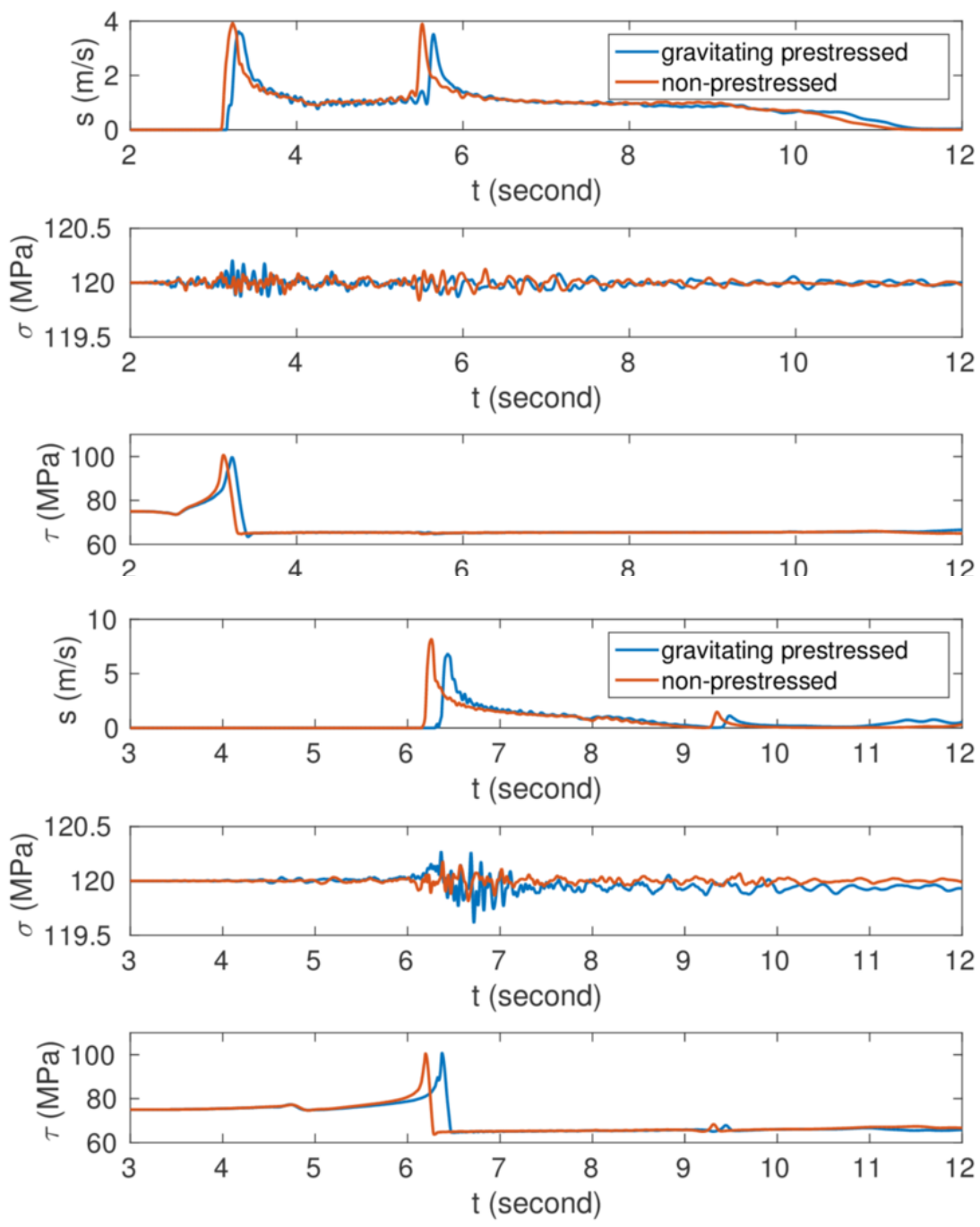

Fig. 27: Comparison of seismograms generated by numerical simulating the planar fault rupturing with and without the consideration of the impacts of static self-gravitation and prestress. The on-fault stations are located at (a) $[0.0,3.0,0.0] \mathrm{km}$, and (b) $[12.0,12.0,0.0] \mathrm{km}$, showing the magnitude of slip rate $s$, compressive normal stress $\sigma$ and magnitude of tangential stress $\tau$ (equals to the friction force $\tau_{\mathrm{f}}$ ),

Here, we show the stability estimate of the spatially discretized and time continuous problem described by Problem 1 in Section 2.4 in the finite-dimensional subspace $V_{1}^{p} \times V_{2}^{p} \times V_{3}^{p}$.

Theorem A.1. If $(\boldsymbol{u}, \boldsymbol{E}, \Psi) \in V_{1}^{p} \times V_{2}^{p} \times V_{3}^{p}$ solves the coupled problem (2.22a)-(2.23d), and if $\gamma$ and $\alpha_{\mathrm{f}}$ satisfy

$$
\gamma>0 \quad \text { and } \quad \alpha_{\mathrm{f}} \geq \max \left(\frac{C_{\boldsymbol{\Lambda}}}{2}, \gamma\right)
$$


then

(A.2)

$$
\begin{gathered}
\|\dot{\boldsymbol{u}}\|_{L^{\infty}\left([0, T] ; L^{2}(\Omega)\right)}^{2}+\|\boldsymbol{u}\|_{L^{\infty}([0, T] ; \mathbb{H})}^{2}+\left\|\boldsymbol{r}_{\mathrm{f}}(\boldsymbol{u})\right\|_{L^{\infty}\left([0, T] ; L^{2}(\Omega)\right)}^{2}+\|\boldsymbol{E}\|_{L^{\infty}\left([0, T] ; L^{2}(\Omega)\right)}^{2}+\|\psi\|_{L^{\infty}\left([0, T] ; L^{2}\left(\Sigma_{\mathrm{f}}\right)\right)}^{2} \\
+\|\dot{u}\|_{L^{2}([0, T] ; \mathbb{H})}^{2}+\left\|\boldsymbol{r}_{\mathrm{f}}(\dot{\boldsymbol{u}})\right\|_{L^{2}\left([0, T] ; L^{2}(\Omega)\right)}^{2}+\|\dot{\boldsymbol{E}}\|_{L^{2}\left([0, T] ; L^{2}(\Omega)\right)}^{2} \leq C
\end{gathered}
$$

Proof. We substitute (2.23a) into (2.22b) and integrate by parts, so that

$$
\int_{\Omega}\left(\dot{\boldsymbol{E}}-\nabla \dot{\boldsymbol{u}}+\boldsymbol{r}_{\mathrm{f}}(\dot{\boldsymbol{u}})\right): \boldsymbol{H} \mathrm{d} \Omega=0
$$

By substituting $\boldsymbol{H}=\dot{\boldsymbol{E}}+\nabla \dot{\boldsymbol{u}}+\boldsymbol{r}_{\mathrm{f}}(\dot{\boldsymbol{u}})$ in (A.3), we obtain, with Young's inequality,

$$
\|\nabla \dot{\boldsymbol{u}}\|_{L^{2}(\Omega)}^{2}=\left\|\dot{\boldsymbol{E}}+\boldsymbol{r}_{\mathrm{f}}(\dot{\boldsymbol{u}})\right\|_{L^{2}(\Omega)}^{2} \leq\left(1+\delta_{1}\right)\|\dot{\boldsymbol{E}}\|_{L^{2}(\Omega)}^{2}+\left(1+\delta_{1}^{-1}\right)\left\|\boldsymbol{r}_{\mathrm{f}}(\dot{\boldsymbol{u}})\right\|_{L^{2}(\Omega)}^{2} .
$$

By substituting $\boldsymbol{H}=\dot{\boldsymbol{E}}-\nabla \dot{\boldsymbol{u}}-\boldsymbol{r}_{\mathrm{f}}(\dot{\boldsymbol{u}})$ in (A.3), we obtain

$$
-\int_{\Omega}(\nabla \dot{\boldsymbol{u}}): \dot{\boldsymbol{E}} \mathrm{d} \Omega+\frac{1}{2}\left(\|\nabla \dot{\boldsymbol{u}}\|_{L^{2}(\Omega)}^{2}+\|\dot{\boldsymbol{E}}\|_{L^{2}(\Omega)}^{2}-\left\|\boldsymbol{r}_{\mathrm{f}}(\dot{\boldsymbol{u}})\right\|_{L^{2}(\Omega)}^{2}\right)=0 .
$$

We integrate (A.3) over time with initial conditions $\left.\boldsymbol{E}\right|_{t=0}=0$ and $\left.\boldsymbol{u}\right|_{t=0}=0$, which yields

$$
\int_{\Omega}\left(\boldsymbol{E}-\nabla \boldsymbol{u}+\boldsymbol{r}_{\mathrm{f}}(\boldsymbol{u})\right): \boldsymbol{H} \mathrm{d} \Omega=0,
$$

and with $\boldsymbol{H}=\boldsymbol{E}+\nabla \boldsymbol{u}+\boldsymbol{r}_{\mathrm{f}}(\boldsymbol{u})$, yields

$$
\|\nabla \boldsymbol{u}\|_{L^{2}(\Omega)}^{2} \leq\left(1+\delta_{2}\right)\|\boldsymbol{E}\|_{L^{2}(\Omega)}^{2}+\left(1+\delta_{2}^{-1}\right)\left\|\boldsymbol{r}_{\mathrm{f}}(\boldsymbol{u})\right\|_{L^{2}(\Omega)}^{2} .
$$

We let $\boldsymbol{w}=\dot{\boldsymbol{u}}, \varphi=\psi$ and $\boldsymbol{H}=\boldsymbol{\Lambda}^{\boldsymbol{T}^{0}}: \boldsymbol{E}$ in (2.22a), (2.22c) and (A.3), and notice that $\boldsymbol{n} \cdot \llbracket \dot{\boldsymbol{u}} \rrbracket=0$. The summation $(2.22 \mathrm{a})+(2.22 \mathrm{c})+($ A.3 $)+($ A.5) yields

$$
\begin{aligned}
\frac{1}{2} \frac{\mathrm{d}}{\mathrm{d} t}( & \left.\|\dot{\boldsymbol{u}}\|_{L^{2}\left(\Omega ; \rho^{0}\right)}^{2}+\int_{\Omega} \boldsymbol{u} \cdot\left(\nabla \nabla \Phi^{0}\right) \cdot \boldsymbol{u} \mathrm{d} \Omega+\|\boldsymbol{E}\|_{L^{2}\left(\Omega ; \boldsymbol{T}^{T^{0}}\right)}^{2}+\alpha_{\mathrm{f}}\left\|\boldsymbol{r}_{\mathrm{f}}(\boldsymbol{u})\right\|_{L^{2}(\Omega)}^{2}+\|\psi\|_{L^{2}\left(\Sigma_{\mathrm{f}}\right)}^{2}\right) \\
& +\frac{\gamma}{2}\left(\|\nabla \dot{\boldsymbol{u}}\|_{L^{2}(\Omega)}^{2}+\|\dot{\boldsymbol{E}}\|_{L^{2}(\Omega)}^{2}+2\|\dot{\boldsymbol{u}}\|_{L^{2}(\Omega)}^{2}\right)+\left(\alpha_{\mathrm{f}}-\frac{\gamma}{2}\right)\left\|\boldsymbol{r}_{\mathrm{f}}(\dot{\boldsymbol{u}})\right\|_{L^{2}(\Omega)}^{2} \\
= & -\int_{\Sigma_{\mathrm{f}}}\left(\boldsymbol{\tau}_{\mathrm{f}}-\left\{\left\{\boldsymbol{\tau}_{2}\right\}\right\}-\boldsymbol{n} \cdot\left(\boldsymbol{T}^{0}+\boldsymbol{T}^{\delta}\right)\right) \cdot \boldsymbol{s} \mathrm{d} \Sigma+\int_{\Sigma_{\mathrm{f}}} \llbracket \boldsymbol{\tau}_{2} \rrbracket \cdot\{\{\dot{\boldsymbol{u}}\}\} \mathrm{d} \Sigma-\int_{\Sigma_{\mathrm{f}}} \mathcal{G}(s, \psi) \psi \mathrm{d} \Sigma .
\end{aligned}
$$

With Young's inequality,

$$
(\mathrm{A} .9)-\int_{\Omega} \boldsymbol{u} \cdot\left(\nabla \nabla \Phi^{0}\right) \cdot \dot{\boldsymbol{u}} \mathrm{d} \Omega \leq C_{\Phi^{0}} \int_{\Omega} \boldsymbol{u} \cdot \dot{\boldsymbol{u}} \mathrm{d} \Omega \leq-\frac{C_{\Phi^{0}}}{2} \frac{\mathrm{d}}{\mathrm{d} t}\|\boldsymbol{u}\|_{L^{2}(\Omega)}^{2}+C_{\Phi^{0}}\left(\|\dot{\boldsymbol{u}}\|_{L^{2}(\Omega)}^{2}+\|\boldsymbol{u}\|_{L^{2}(\Omega)}^{2}\right) .
$$

We mention some results from [66, Section 4.2],

$$
\begin{aligned}
& \int_{\Omega}\left(\left\{\left\{\boldsymbol{\tau}_{2}\right\}\right\} \cdot s+\llbracket \boldsymbol{\tau}_{2} \rrbracket \cdot\{\{\dot{\boldsymbol{u}}\}\}\right) \mathrm{d} \Omega \leq C_{I}^{\prime}\left(\frac{1}{2 \delta_{3}}\|\boldsymbol{u}\|_{\mathbb{H}}^{2}+\frac{\delta_{3}}{2}\|\dot{\boldsymbol{u}}\|_{\mathbb{H}}^{2}\right), \\
& -\int_{\Sigma_{\mathrm{f}}} \mathcal{G}(s, \psi) \psi \mathrm{d} \Sigma \leq C_{\mathcal{G}_{0}}+\left(\frac{C_{\mathcal{G}, s}^{\star}}{2 \delta_{4}}-C_{\mathcal{G}, \psi}\right)\|\psi\|_{L^{2}\left(\Sigma_{\mathrm{f}}\right)}^{2}+\frac{C_{\mathcal{G}, s}^{\star} \delta_{4}}{2}\|s\|_{L^{2}\left(\Sigma_{\mathrm{f}}\right)}^{2},
\end{aligned}
$$


where the constant $C_{\mathcal{G}_{0}}$ depends on the initial value, $\mathcal{G}\left(\left.s\right|_{t=0}, \psi_{t=0}\right)$. With Young's inequality,

$$
\int_{\Sigma_{\mathrm{f}}} \boldsymbol{n} \cdot\left(\boldsymbol{T}^{0}+\boldsymbol{T}^{\delta}\right) \cdot \boldsymbol{s} \mathrm{d} \Sigma \leq \frac{1}{\delta}\left\|\boldsymbol{n} \cdot\left(\boldsymbol{T}^{0}+\boldsymbol{T}^{\delta}\right)\right\|_{L^{2}\left(\Sigma_{\mathrm{f}}\right)}^{2}+\delta_{4}\|s\|_{L^{2}\left(\Sigma_{\mathrm{f}}\right)}^{2} .
$$

With (2.9) implying $\boldsymbol{\tau}_{\mathrm{f}} \cdot s=\tau_{\mathrm{f}} s>0$, we let $\left(1+\frac{C_{\mathcal{G}, s}^{\star}}{2}\right) \delta_{4}=\left(\int_{\Sigma_{\mathrm{f}}} \tau_{\mathrm{f}} s \mathrm{~d} \Sigma\right) /\|s\|_{L^{2}\left(\Sigma_{\mathrm{f}}\right)}^{2} \geq C_{\mathcal{F}, s}$ therefore,

$$
\begin{aligned}
-\int_{\Sigma_{\mathrm{f}}}\left(\boldsymbol{\tau}_{\mathrm{f}}-\boldsymbol{n} \cdot\left(\boldsymbol{T}^{0}+\boldsymbol{T}^{\delta}\right)\right) \cdot \boldsymbol{s} \mathrm{d} \Sigma-\int_{\Sigma_{\mathrm{f}}} \mathcal{G}(s, \psi) \psi \mathrm{d} \Sigma \\
\leq C+\frac{1}{\delta_{4}}\left\|\boldsymbol{n} \cdot\left(\boldsymbol{T}^{0}+\boldsymbol{T}^{\delta}\right)\right\|_{L^{2}\left(\Sigma_{\mathrm{f}}\right)}^{2}+\left(\frac{C_{\mathcal{G}, s}^{\star}}{2 \delta_{4}}-C_{\mathcal{G}, \psi}\right)\|\psi\|_{L^{2}\left(\Sigma_{\mathrm{f}}\right)}^{2} \\
\quad+\delta_{4}\left(1+\frac{C_{\mathcal{G}, s}^{\star}}{2}\right)\|s\|_{L^{2}\left(\Sigma_{\mathrm{f}}\right)}^{2}-\int_{\Sigma_{\mathrm{f}}} \tau_{\mathrm{f}} s \mathrm{~d} \Sigma \\
\leq C+\left(\frac{C_{\mathcal{G}, s}^{\star}\left(2+C_{\mathcal{G}, s}^{\star}\right)}{4 C_{\mathcal{F}, s}}-C_{\mathcal{G}, \psi}\right)\|\psi\|_{L^{2}\left(\Sigma_{\mathrm{f}}\right)}^{2}+\frac{2+C_{\mathcal{G}, s}^{\star}}{2 C_{\mathcal{F}, s}}\left\|\boldsymbol{n} \cdot\left(\boldsymbol{T}^{0}+\boldsymbol{T}^{\delta}\right)\right\|_{L^{2}\left(\Sigma_{\mathrm{f}}\right)}^{2}
\end{aligned}
$$

We substitute (A.4), (A.7) and (A.9) into (A.8) and let $\delta_{1}=\delta_{2}=1$, use the results in (A.10)(A.13) with $\delta_{3}=\frac{\gamma}{2 C_{I}^{\prime}}$ in (A.10), and integrate over time $[0, t]$ for $t \leq T$ to find the energy estimate

$$
\begin{aligned}
& \frac{1}{2}\left(C_{\rho^{0}}\|\dot{\boldsymbol{u}}\|_{L^{2}(\Omega)}^{2}+C_{\phi^{0}}\|\boldsymbol{u}\|_{L^{2}(\Omega)}^{2}+\frac{C_{\boldsymbol{\Lambda}}}{2}\|\boldsymbol{E}\|_{L^{2}(\Omega)}^{2}+\frac{C_{\boldsymbol{\Lambda}}}{4}\|\nabla \boldsymbol{u}\|_{L^{2}(\Omega)}^{2}+\left(\alpha_{\mathrm{f}}-\frac{C_{\boldsymbol{\Lambda}}}{2}\right)\left\|\boldsymbol{r}_{\mathrm{f}}(\boldsymbol{u})\right\|_{L^{2}(\Omega)}^{2}+\|\psi\|_{L^{2}\left(\Sigma_{\mathrm{f}}\right)}^{2}\right) \\
& \quad+\int_{0}^{t}\left(\frac{\gamma}{2}\|\dot{\boldsymbol{E}}\|_{L^{2}(\Omega)}^{2}+\frac{\gamma}{4}\|\dot{\boldsymbol{u}}\|_{\mathbb{H}}^{2}+\left(\alpha_{\mathrm{f}}-\gamma\right)\left\|\boldsymbol{r}_{\mathrm{f}}(\dot{\boldsymbol{u}})\right\|_{L^{2}(\Omega)}^{2}\right) \mathrm{d} \tau \\
& \leq \int_{0}^{t}\left(C_{\mathcal{G}_{0}}+\frac{2+C_{\mathcal{G}, s}^{\star}}{2 C_{\mathcal{F}, s}}\left\|\boldsymbol{n} \cdot\left(\boldsymbol{T}^{0}+\boldsymbol{T}^{\delta}\right)\right\|_{L^{2}\left(\Sigma_{\mathrm{f}}\right)}^{2}+\frac{C_{I}^{\prime}}{\gamma}\|\boldsymbol{u}\|_{\mathbb{H}}^{2}\right. \\
& \left.\quad+C_{\phi^{0}}\left(\|\dot{\boldsymbol{u}}\|_{L^{2}(\Omega)}^{2}+\|\boldsymbol{u}\|_{L^{2}(\Omega)}^{2}\right)+\left(\frac{C_{\mathcal{G}, s}^{\star}\left(2+C_{\mathcal{G}, s}^{\star}\right)}{4 C_{\mathcal{F}, s}}-C_{\mathcal{G}, \psi}\right)\|\psi\|_{L^{2}\left(\Sigma_{\mathrm{f}}\right)}^{2}\right) \mathrm{d} \tau .
\end{aligned}
$$

We assume (A.1) holds, and based on Gronwall's lemma,

(A.15)

$$
\begin{aligned}
& \|\dot{\boldsymbol{u}}\|_{L^{2}(\Omega)}^{2}+\|\boldsymbol{u}\|_{\mathbb{H}^{-}}^{2}+\|\boldsymbol{E}\|_{L^{2}(\Omega)}^{2}+\left\|\boldsymbol{r}_{\mathrm{f}}(\boldsymbol{u})\right\|_{L^{2}(\Omega)}^{2}+\|\psi\|_{L^{2}\left(\Sigma_{\mathrm{f}}\right)}^{2} \\
& \quad+\|\dot{\boldsymbol{E}}\|_{L^{2}\left([0, t] ; L^{2}(\Omega)\right)}^{2}+\|\dot{\boldsymbol{u}}\|_{L^{2}([0, t] ; \mathbb{H})}^{2}+\left\|\boldsymbol{r}_{\mathrm{f}}(\dot{\boldsymbol{u}})\right\|_{L^{2}\left([0, t] ; L^{2}(\Omega)\right)}^{2} \leq C \exp \left(\left\|\boldsymbol{n} \cdot\left(\boldsymbol{T}^{0}+\boldsymbol{T}^{\delta}\right)\right\|_{L^{2}\left([0, t], L^{2}\left(\Sigma_{\mathrm{f}}\right)\right)}^{2}\right) .
\end{aligned}
$$

Since (A.15) is satisfied at any time $t \in[0, T],($ A.2) holds.

Remark 2. In the above estimate, we use strict positivity of $\gamma$. As Problem 1 yields a regularization of the original problem, we will limit coefficient $\gamma$ to be sufficiently small, so that the regularized problem approaches the original one. In practice, it is sufficient to have strictly positive $\gamma$ only in the vicinity of the rupture region (see Section 5).

\section{Appendix B. Stability of the iterative coupling.}

We prove that the iterative coupling described in Section 4 is a contraction under certain constraints on model coefficients, in parallel with the stability result for the second-order formulation of motion in Ye, et al. (2019)[66, Section 6]. In contrast to the reference, we show this contraction for the numerical scheme presented here, in particular, based on a mixed strain-velocity formulation using the DG spatial discretization. For simplicity, we take the same time step for the wave equation and the state ODE, that is, a single-rate scheme. The proof is obtained along the lines of the reference. 
TheOREm B.1. The iterative coupling scheme (4.1a)-(4.1c) is a contraction within each time step in the sense that

$$
\begin{gathered}
\sum_{\Omega^{\mathrm{e}}}\left(C_{1}\left\|\boldsymbol{\epsilon}_{\boldsymbol{v}}^{k}\right\|_{L^{2}\left(\Omega^{\mathrm{e}}\right)}^{2}+C_{2}\left\|\nabla \boldsymbol{\epsilon}_{\boldsymbol{v}}^{k}\right\|_{L^{2}\left(\Omega^{\mathrm{e}}\right)}^{2}+C_{3}\left\|\boldsymbol{\epsilon}_{\boldsymbol{E}}^{k}\right\|_{L^{2}\left(\Omega^{\mathrm{e}}\right)}^{2}\right)+C_{4} \sum_{\Sigma_{\mathrm{f}}^{\mathrm{e}}}\left\|\epsilon_{\psi}^{k}\right\|_{L^{2}\left(\Sigma_{\mathrm{f}}^{\mathrm{e}}\right)}^{2} \\
<\lambda\left(C_{1}\left\|\boldsymbol{\epsilon}_{\boldsymbol{v}}^{k-1}\right\|_{L^{2}\left(\Omega^{\mathrm{e}}\right)}^{2}+C_{3}\left\|\boldsymbol{\epsilon}_{\boldsymbol{E}}^{k-1}\right\|_{L^{2}\left(\Omega^{\mathrm{e}}\right)}^{2}\right)
\end{gathered}
$$

where

$$
\boldsymbol{\epsilon}_{\boldsymbol{v}}^{k}:=\boldsymbol{v}_{h}^{(n, k)}-\boldsymbol{v}_{h}^{(n, k-1)}, \quad \boldsymbol{\epsilon}_{\boldsymbol{E}}^{k}:=\boldsymbol{E}_{h}^{(n, k)}-\boldsymbol{E}_{h}^{(n, k-1)}, \quad \epsilon_{\psi}^{k}:=\psi_{h}^{(n, k)}-\psi_{h}^{(n, k-1)},
$$

and with $C_{1}, C_{2}, C_{3}, C_{4}>0$ and $\lambda \in(0,1)$ some constants, if $\gamma^{\mathrm{e}}, \alpha_{\mathrm{f}}^{\mathrm{e}}, \alpha_{\mathrm{c}}^{\mathrm{e}}$ and $\Delta t$ satisfy

$$
\begin{aligned}
& \frac{1}{\Delta t}>\frac{C_{\mathcal{F}, \psi}^{\star 2}}{2 C_{\mathcal{F}, s}}+\frac{C_{\mathcal{G}, s}^{\star 2}}{2 C_{\mathcal{F}, s}}-C_{\mathcal{G}, \psi}, \\
& \frac{\gamma^{\mathrm{e}}}{\Delta t}>\max \left(2\left(C_{\mathcal{F}, \sigma}^{\star}\left(C_{I}+C_{I}^{\prime}\right)+C_{I}^{\prime}\right), \quad \frac{\Delta t\left(C_{\boldsymbol{\Lambda}}^{\star}\right)^{2}}{2 \lambda h}-C_{\boldsymbol{\Lambda}}+\sqrt{\left(\frac{\Delta t\left(C_{\boldsymbol{\Lambda}}^{\star}\right)^{2}}{2 \lambda h}-C_{\boldsymbol{\Lambda}}\right)^{2}-\left(C_{\boldsymbol{\Lambda}}^{\star}\right)^{2}}\right), \\
& \alpha_{\mathrm{c}}^{\mathrm{e}}<\frac{\lambda h}{2(1+\lambda) C_{p}}\left(\frac{C_{\rho^{0}}}{\Delta t}-\Delta t C_{\phi^{0}}+\frac{\gamma^{\mathrm{e}}}{2}-\frac{2 C_{p} C_{r}^{\star}}{h}\right), \\
& \alpha_{\mathrm{f}}^{\mathrm{e}}>C_{r}^{\star}\left(\frac{4(\Delta t+1) h}{\Delta t\left(C_{\boldsymbol{\Lambda}}^{\star}\right)^{2}}\left(C_{\boldsymbol{\Lambda}}+\frac{\gamma^{\mathrm{e}}}{2 \Delta t}\right)-\frac{4(\Delta t+1) h}{2 \lambda h}-\frac{4(\Delta t+1) h}{2 \gamma^{\mathrm{e}}}\right)^{-1} .
\end{aligned}
$$

Proof. The proof here assumes a fixed single-rate time step $\Delta t$ and a fixed mesh size $h$. We define

$$
\begin{aligned}
& \epsilon_{\boldsymbol{\tau}_{2}}^{k}:=\boldsymbol{\tau}_{2 h}^{(n, k)}-\boldsymbol{\tau}_{2 h}^{(n, k-1)}, \quad \epsilon_{\boldsymbol{\tau}_{\mathrm{f}}}^{k}:=\boldsymbol{\tau}_{\mathrm{f} h}^{(n, k)}-\boldsymbol{\tau}_{\mathrm{f} h}^{(n, k-1)}, \quad \boldsymbol{\epsilon}_{\boldsymbol{s}}^{k}:=\boldsymbol{s}_{h}^{(n, k)}-\boldsymbol{s}_{h}^{(n, k-1)}, \\
& \epsilon_{\sigma}^{k}:=\sigma_{h}^{(n, k)}-\sigma_{h}^{(n, k-1)}, \quad \epsilon_{\mathcal{F}}^{k}:=\mathcal{F}\left(\sigma_{h}^{(n, k)}, s_{h}^{(n, k)}, \psi_{h}^{(n, k)}\right)-\mathcal{F}\left(\sigma_{h}^{(n, k-1)}, s_{h}^{(n, k-1)}, \psi_{h}^{(n, k-1)}\right), \\
& \epsilon_{\mathcal{G}}^{k}:=\mathcal{G}\left(s_{h}^{(n, k)}, \psi_{h}^{(n, k)}\right)-\mathcal{G}\left(s_{h}^{(n, k-1)}, \psi_{h}^{(n, k-1)}\right), \quad \epsilon_{s}^{k}:=\left|\boldsymbol{s}_{h}^{(n, k)}\right|-\left|\boldsymbol{s}_{h}^{(n, k-1)}\right| .
\end{aligned}
$$

We eliminate $\boldsymbol{u}_{h}^{(n, k)}$ by $\boldsymbol{u}_{h}^{(n, k)}-\Delta t \boldsymbol{v}_{h}^{(n, k)}=\boldsymbol{u}_{h}^{(n-1)}$ in (4.2a), and subtract iteration $k$ from iteration $k-1$ of $(4.1 \mathrm{a}-\mathrm{d})$ to obtain the error estimate:

$$
\begin{aligned}
\sum_{\Omega^{\mathrm{e}}} & \int_{\Omega^{\mathrm{e}}}\left(\left(\rho_{h}^{0}\left(\frac{1}{\Delta t} \boldsymbol{\epsilon}_{\boldsymbol{v}}^{k}+\Delta t \boldsymbol{\epsilon}_{\boldsymbol{v}}^{k} \cdot \boldsymbol{K}_{h}\right)+\gamma^{\mathrm{e}} \boldsymbol{\epsilon}_{\boldsymbol{v}}^{k}\right) \cdot \boldsymbol{w}_{h}+\left(\boldsymbol{\Lambda}_{h}^{\boldsymbol{T}^{0}}: \boldsymbol{\epsilon}_{\boldsymbol{E}}^{k}+\frac{\gamma^{\mathrm{e}}}{\Delta t} \boldsymbol{\epsilon}_{\boldsymbol{E}}^{k}\right): \nabla \boldsymbol{w}_{h}\right) \mathrm{d} \Omega \\
& +\sum_{\Sigma_{\mathrm{f}}^{\mathrm{e}}} \int_{\Sigma_{\mathrm{f}}^{\mathrm{e}}}\left(\left(\boldsymbol{\epsilon}_{\boldsymbol{\tau}_{\mathrm{f}}}^{k}-\epsilon_{\sigma}^{k} \boldsymbol{n}\right) \cdot \llbracket \boldsymbol{w}_{h} \rrbracket-\llbracket \boldsymbol{\epsilon}_{\boldsymbol{\tau}_{2}}^{k} \cdot \boldsymbol{w}_{h} \rrbracket+(\Delta t+1) \alpha_{\mathrm{f}}^{\mathrm{e}} \llbracket \boldsymbol{n} \cdot \boldsymbol{\epsilon}_{\boldsymbol{v}}^{k} \rrbracket \llbracket \boldsymbol{n} \cdot \boldsymbol{w}_{h} \rrbracket\right) \mathrm{d} \Sigma \\
= & -\sum_{\Sigma_{\mathrm{c}}^{\mathrm{e}}} \int_{\Sigma_{\mathrm{c}}^{\mathrm{e}}}\left(\left\{\left\{\boldsymbol{n} \cdot\left(\boldsymbol{\Lambda}_{h}^{\boldsymbol{T}^{0}}: \boldsymbol{\epsilon}_{\boldsymbol{E}}^{k-1}\right)\right\}\right\}+\alpha_{\mathrm{c}}^{\mathrm{e}} \llbracket \boldsymbol{\epsilon}_{\boldsymbol{v}}^{k-1} \rrbracket\right) \cdot \llbracket \boldsymbol{w}_{h} \rrbracket \mathrm{d} \Sigma
\end{aligned}
$$

$$
\begin{gathered}
\sum_{\Omega^{\mathrm{e}}} \int_{\Omega^{\mathrm{e}}}\left(\frac{1}{\Delta t} \boldsymbol{\epsilon}_{\boldsymbol{E}}^{k}: \boldsymbol{H}_{h} \mathrm{~d} \Omega+\boldsymbol{\epsilon}_{\boldsymbol{v}}^{k} \cdot\left(\nabla \cdot \boldsymbol{H}_{h}\right)\right) \mathrm{d} \Omega+\sum_{\Sigma_{\mathrm{f}}^{\mathrm{e}}} \int_{\Sigma_{\mathrm{f}}^{\mathrm{e}}}\left(\left\{\left\{\boldsymbol{\epsilon}_{\boldsymbol{v}}^{k}\right\}\right\} \cdot \llbracket \boldsymbol{n} \cdot \boldsymbol{H}_{h} \rrbracket+\boldsymbol{\epsilon}_{\boldsymbol{s}}^{k} \cdot\left\{\left\{\boldsymbol{n} \cdot \boldsymbol{H}_{h}\right\}\right\}\right) \mathrm{d} \Sigma \\
=-\sum_{\Sigma_{\mathrm{c}}^{\mathrm{e}}} \int_{\Sigma_{\mathrm{c}}^{\mathrm{e}}}\left(\left\{\left\{\boldsymbol{\epsilon}_{\boldsymbol{v}}^{k-1}\right\}\right\}+\alpha_{\mathrm{c}}^{\mathrm{e}} \llbracket \boldsymbol{n} \cdot\left(\boldsymbol{\Lambda}_{h}^{\boldsymbol{T}^{0}}: \boldsymbol{\epsilon}_{\boldsymbol{E}}^{k-1}\right) \rrbracket\right) \cdot \llbracket \boldsymbol{n} \cdot \boldsymbol{H}_{h} \rrbracket \mathrm{d} \Sigma
\end{gathered}
$$


Integrating (B.3b) by parts yields

$$
\begin{gathered}
\sum_{\Omega^{\mathrm{e}}} \int_{\Omega^{\mathrm{e}}}\left(\frac{1}{\Delta t} \boldsymbol{\epsilon}_{\boldsymbol{E}}^{k}-\nabla \boldsymbol{\epsilon}_{\boldsymbol{v}}^{k}\right): \boldsymbol{H}_{h} \mathrm{~d} \Omega-\sum_{\Sigma_{\mathrm{f}}^{\mathrm{e}}} \int_{\Sigma_{\mathrm{f}}^{\mathrm{e}}} \llbracket \boldsymbol{n} \cdot \boldsymbol{\epsilon}_{\boldsymbol{v}}^{k} \rrbracket\left\{\left\{\boldsymbol{n} \cdot \boldsymbol{H}_{h} \cdot \boldsymbol{n}\right\}\right\} \mathrm{d} \Sigma \\
=\sum_{\Sigma_{\mathrm{c}}^{\mathrm{e}}} \int_{\Sigma_{\mathrm{c}}^{\mathrm{e}}}\left\{\left\{\boldsymbol{\epsilon}_{\boldsymbol{v}}^{k}-\boldsymbol{\epsilon}_{\boldsymbol{v}}^{k-1}\right\}\right\} \llbracket \boldsymbol{n} \cdot \boldsymbol{H}_{h} \rrbracket \mathrm{d} \Sigma+\sum_{\Sigma_{\mathrm{c}}^{\mathrm{e}}} \int_{\Sigma_{\mathrm{c}}^{\mathrm{e}}} \llbracket \boldsymbol{\epsilon}_{\boldsymbol{v}}^{k} \rrbracket\left\{\left\{\boldsymbol{n} \cdot \boldsymbol{H}_{h}\right\}\right\} \mathrm{d} \Sigma \\
-\sum_{\Sigma_{\mathrm{c}}^{\mathrm{e}}} \int_{\Sigma_{\mathrm{c}}^{\mathrm{e}}} \alpha_{\mathrm{c}}^{\mathrm{e}} \llbracket \boldsymbol{n} \cdot\left(\boldsymbol{\Lambda}_{h}^{\boldsymbol{T}^{0}}: \boldsymbol{\epsilon}_{\boldsymbol{E}}^{k-1}\right) \rrbracket \llbracket \boldsymbol{n} \cdot \boldsymbol{H}_{h} \rrbracket \mathrm{d} \Sigma
\end{gathered}
$$

We define linear continuous maps ("lifting operators", see Arnold et al. (2002) [7]), $\boldsymbol{r}_{\mathrm{f}}^{\mathrm{e}}: L^{2}\left(\Sigma_{\mathrm{f}}^{\mathrm{e}}\right) \rightarrow$ $\hat{V}_{2 h}^{p}, \boldsymbol{r}_{1}^{\mathrm{e}}: L^{2}\left(\Sigma_{\mathrm{c}}^{\mathrm{e}}\right) \rightarrow \hat{V}_{2 h}^{p}$, and $\boldsymbol{r}_{2}^{\mathrm{e}}: L^{2}\left(\Sigma_{\mathrm{c}}^{\mathrm{e}}\right) \rightarrow \hat{V}_{2 h}^{p}$, with $\hat{V}_{2 h}^{p}$ defined in (3.3), such that

$$
\begin{aligned}
& \int_{\Omega^{\mathrm{e}^{ \pm}}} \boldsymbol{r}_{\mathrm{f}}^{\mathrm{e}}(v): \boldsymbol{H}_{h} \mathrm{~d} \Omega=\int_{\Sigma_{\mathrm{f}}^{\mathrm{e}}} v\left\{\left\{\boldsymbol{n} \cdot \boldsymbol{H}_{h} \cdot \boldsymbol{n}\right\}\right\} \mathrm{d} \Sigma, \quad \text { for } \Sigma_{\mathrm{f}}^{\mathrm{e}}=\Omega^{\mathrm{e}^{+}} \cap \Omega^{\mathrm{e}^{-}}, \\
& \int_{\Omega^{\mathrm{e}^{ \pm}}} \boldsymbol{r}_{1}^{\mathrm{e}}(\boldsymbol{v}): \boldsymbol{H}_{h} \mathrm{~d} \Omega=\int_{\Sigma_{\mathrm{c}}^{\mathrm{e}}} \boldsymbol{v} \cdot\left\{\left\{\boldsymbol{n} \cdot \boldsymbol{H}_{h}\right\}\right\} \mathrm{d} \Sigma, \quad \text { for } \Sigma_{\mathrm{c}}^{\mathrm{e}}=\Omega^{\mathrm{e}^{+}} \cap \Omega^{\mathrm{e}^{-}} . \\
& \int_{\Omega^{\mathrm{e}} \pm} \boldsymbol{r}_{2}^{\mathrm{e}}(\boldsymbol{v}): \boldsymbol{H}_{h} \mathrm{~d} \Omega=\int_{\Sigma_{\mathrm{c}}^{\mathrm{e}}} \boldsymbol{v} \cdot \llbracket \boldsymbol{n} \cdot \boldsymbol{H}_{h} \rrbracket \mathrm{d} \Sigma, \quad \text { for } \Sigma_{\mathrm{c}}^{\mathrm{e}}=\Omega^{\mathrm{e}^{+}} \cap \Omega^{\mathrm{e}^{-}} .
\end{aligned}
$$

It is suggested in Arnold et al. (2002) [7] that there exist constants $C_{r}, C_{r}^{\star}>0$ such that

$$
\begin{aligned}
& C_{r} h^{-1}\|v\|_{L^{2}\left(\Sigma_{\mathrm{f}}^{\mathrm{e}}\right)}^{2} \leq\left\|\boldsymbol{r}_{\mathrm{f}}^{\mathrm{e}}(v)\right\|_{L^{2}\left(\Omega^{\mathrm{e}^{ \pm}}\right)}^{2} \leq C_{r}^{\star} h^{-1}\|v\|_{L^{2}\left(\Sigma_{\mathrm{f}}^{\mathrm{e}}\right)}^{2}, \\
& C_{r} h^{-1}\|\boldsymbol{v}\|_{L^{2}\left(\Sigma_{\mathrm{c}}^{\mathrm{e}}\right)}^{2} \leq\left\|\boldsymbol{r}_{1}^{\mathrm{e}}(\boldsymbol{v})\right\|_{L^{2}\left(\Omega^{\mathrm{e}^{ \pm}}\right)}^{2} \leq C_{r}^{\star} h^{-1}\|\boldsymbol{v}\|_{L^{2}\left(\Sigma_{\mathrm{c}}^{\mathrm{e}}\right)}^{2}, \\
& C_{r} h^{-1}\|\boldsymbol{v}\|_{L^{2}\left(\Sigma_{\mathrm{c}}^{\mathrm{e}}\right)}^{2} \leq\left\|\boldsymbol{r}_{2}^{\mathrm{e}}(\boldsymbol{v})\right\|_{L^{2}\left(\Omega^{\mathrm{e}^{ \pm}}\right)}^{2} \leq C_{r}^{\star} h^{-1}\|\boldsymbol{v}\|_{L^{2}\left(\Sigma_{\mathrm{c}}^{\mathrm{e}}\right)}^{2},
\end{aligned}
$$

and in Warburton and Hesthaven (2003) [60] that there exist a constant $C_{p}>0$ such that

$$
\|v\|_{L^{2}\left(\partial \Omega^{\mathrm{e}}\right)}^{2} \leq C_{p} h^{-1}\|v\|_{L^{2}\left(\Omega^{\mathrm{e}}\right)}^{2}
$$

if $\Omega^{\mathrm{e}}$ is a tetrahedral element, and $C_{p}=\mathcal{O}\left(p^{2}\right)$ with polynomial order $p$. Based on (B.4) and (B.5),

$$
\begin{aligned}
\int_{\Omega^{\mathrm{e}}}\left(\frac{1}{\Delta t} \boldsymbol{\epsilon}_{\boldsymbol{E}}^{k}\right. & -\nabla \boldsymbol{\epsilon}_{\boldsymbol{v}}^{k}+\boldsymbol{r}_{\mathrm{f}}^{\mathrm{e}}\left(\llbracket \boldsymbol{n} \cdot \boldsymbol{\epsilon}_{\boldsymbol{v}}^{k} \rrbracket\right) \\
& \left.-\boldsymbol{r}_{1}^{\mathrm{e}}\left(\llbracket \boldsymbol{\epsilon}_{\boldsymbol{v}}^{k} \rrbracket\right)-\boldsymbol{r}_{2}^{\mathrm{e}}\left(\left\{\left\{\boldsymbol{\epsilon}_{\boldsymbol{v}}^{k}-\boldsymbol{\epsilon}_{\boldsymbol{v}}^{k-1}\right\}\right\}\right)+\alpha_{\mathrm{c}}^{\mathrm{e}} \boldsymbol{r}_{2}^{\mathrm{e}}\left(\llbracket \boldsymbol{n} \cdot\left(\boldsymbol{\Lambda}_{h}^{\boldsymbol{T}^{0}}: \boldsymbol{\epsilon}_{\boldsymbol{E}}^{k-1}\right) \rrbracket\right)\right): \boldsymbol{H}_{h} \mathrm{~d} \Omega=0,
\end{aligned}
$$

and by taking $\boldsymbol{H}=-\frac{1}{\Delta t} \boldsymbol{\epsilon}_{\boldsymbol{E}}^{k}+\nabla \boldsymbol{\epsilon}_{\boldsymbol{v}}^{k}+\boldsymbol{r}_{\mathrm{f}}^{\mathrm{e}}\left(\llbracket \boldsymbol{n} \cdot \boldsymbol{\epsilon}_{\boldsymbol{v}}^{k} \rrbracket\right)-\boldsymbol{r}_{1}^{\mathrm{e}}\left(\llbracket \boldsymbol{\epsilon}_{\boldsymbol{v}}^{k} \rrbracket\right)-\boldsymbol{r}_{2}^{\mathrm{e}}\left(\left\{\left\{\boldsymbol{\epsilon}_{\boldsymbol{v}}^{k}-\boldsymbol{\epsilon}_{\boldsymbol{v}}^{k-1}\right\}\right\}\right)+\alpha_{\mathrm{c}}^{\mathrm{e}} \boldsymbol{r}_{2}^{\mathrm{e}}\left(\llbracket \boldsymbol{n} \cdot\left(\boldsymbol{\Lambda}_{h}^{\boldsymbol{T}^{0}}: \boldsymbol{\epsilon}_{\boldsymbol{E}}^{k-1}\right) \rrbracket\right)$ in (B.8), we obtain

$$
\begin{aligned}
& \sum_{\Omega^{\mathrm{e}}} \int_{\Omega^{\mathrm{e}}} \boldsymbol{\epsilon}_{\boldsymbol{E}}^{k}: \nabla \boldsymbol{\epsilon}_{\boldsymbol{v}}^{k} \mathrm{~d} \Omega=\frac{1}{2}\left(\Delta t \sum_{\Omega^{\mathrm{e}}}\left\|\nabla \boldsymbol{\epsilon}_{\boldsymbol{v}}^{k}\right\|_{L^{2}\left(\Omega^{\mathrm{e}}\right)}^{2}+\frac{1}{\Delta t} \sum_{\Omega^{\mathrm{e}}}\left\|\boldsymbol{\epsilon}_{\boldsymbol{E}}^{k}\right\|_{L^{2}\left(\Omega^{\mathrm{e}}\right)}^{2}\right) \\
& \quad+\frac{\Delta t}{2} \int_{\Omega^{\mathrm{e}}}\left(\boldsymbol{r}_{\mathrm{f}}^{\mathrm{e}}\left(\llbracket \boldsymbol{n} \cdot \boldsymbol{\epsilon}_{\boldsymbol{v}}^{k} \rrbracket\right)-\boldsymbol{r}_{1}^{\mathrm{e}}\left(\llbracket \boldsymbol{\epsilon}_{\boldsymbol{v}}^{k} \rrbracket\right)-\boldsymbol{r}_{2}^{\mathrm{e}}\left(\left\{\left\{\boldsymbol{\epsilon}_{\boldsymbol{v}}^{k}-\boldsymbol{\epsilon}_{\boldsymbol{v}}^{k-1}\right\}\right\}\right)+\alpha_{\mathrm{c}}^{\mathrm{e}} \boldsymbol{r}_{2}^{\mathrm{e}}\left(\llbracket \boldsymbol{n} \cdot\left(\boldsymbol{\Lambda}_{h}^{\boldsymbol{T}^{0}}: \boldsymbol{\epsilon}_{\boldsymbol{E}}^{k-1}\right) \rrbracket\right)\right)^{2} \mathrm{~d} \Omega
\end{aligned}
$$


Similar to (A.8), we let $\boldsymbol{w}_{h}=\boldsymbol{\epsilon}_{\boldsymbol{v}}^{k}$ in (B.3a) and $\boldsymbol{H}_{h}=\boldsymbol{\Lambda}_{h}^{\boldsymbol{T}^{0}}: \boldsymbol{\epsilon}_{\boldsymbol{E}}^{k}$ in (B.4), and summarize (B.3a) and (B.4) to obtain

(B.10)

$$
\begin{aligned}
\sum_{\Omega^{\mathrm{e}}}( & \left.\frac{1}{\Delta t}\left\|\boldsymbol{\epsilon}_{\boldsymbol{v}}^{k}\right\|_{L^{2}\left(\Omega^{\mathrm{e}} ; \rho_{h}^{0}\right)}^{2}+\Delta t \int_{\Omega^{\mathrm{e}}} \boldsymbol{\epsilon}_{\boldsymbol{v}}^{k} \cdot \boldsymbol{K}_{h} \cdot \boldsymbol{\epsilon}_{\boldsymbol{v}}^{k} \mathrm{~d} \Omega+\gamma^{\mathrm{e}}\left\|\boldsymbol{\epsilon}_{\boldsymbol{v}}^{k}\right\|_{L^{2}\left(\Omega^{\mathrm{e}}\right)}^{2}+\frac{1}{\Delta t}\left\|\boldsymbol{\epsilon}_{\boldsymbol{E}}^{k}\right\|_{L^{2}\left(\Omega^{\mathrm{e}} ; \boldsymbol{\Lambda}_{h}^{T^{0}}\right)}^{2}\right) \\
& +\sum_{\Omega^{\mathrm{e}}} \frac{\gamma^{\mathrm{e}}}{\Delta t} \int_{\Omega^{\mathrm{e}}}\left(\boldsymbol{\epsilon}_{\boldsymbol{E}}^{k}: \nabla \boldsymbol{\epsilon}_{\boldsymbol{v}}^{k}\right) \mathrm{d} \Omega+\sum_{\Sigma_{\mathrm{f}}^{\mathrm{e}}} \alpha_{\mathrm{f}}^{\mathrm{e}}(\Delta t+1)\left\|\llbracket \boldsymbol{n} \cdot \boldsymbol{\epsilon}_{\boldsymbol{v}}^{k} \rrbracket\right\|_{L^{2}\left(\Sigma_{\mathrm{f}}^{\mathrm{e}}\right)}^{2} \\
& +\sum_{\Sigma_{\mathrm{f}}^{\mathrm{e}}} \int_{\Sigma_{\mathrm{f}}^{\mathrm{e}}}\left(\boldsymbol{\epsilon}_{\boldsymbol{\tau}_{\mathrm{f}}^{k}}^{k} \cdot \boldsymbol{\epsilon}_{\boldsymbol{s}}^{k}-\left\{\left\{\boldsymbol{\epsilon}_{\boldsymbol{\tau}_{2}}^{k}\right\}\right\} \cdot \boldsymbol{\epsilon}_{\boldsymbol{s}}^{k}-\llbracket \boldsymbol{\epsilon}_{\boldsymbol{\tau}_{2}}^{k} \rrbracket \cdot\left\{\left\{\boldsymbol{\epsilon}_{\boldsymbol{v}}^{k}\right\}\right\}\right) \mathrm{d} \Sigma \\
= & -\sum_{\Sigma_{\mathrm{c}}^{\mathrm{e}}} \int_{\Sigma_{\mathrm{c}}^{\mathrm{e}}} \alpha_{\mathrm{c}}^{\mathrm{e}} \llbracket \boldsymbol{\epsilon}_{\boldsymbol{v}}^{k-1} \rrbracket \cdot \llbracket \boldsymbol{\epsilon}_{\boldsymbol{v}}^{k} \rrbracket \mathrm{d} \Sigma-\sum_{\Sigma_{\mathrm{c}}^{\mathrm{e}}} \int_{\Sigma_{\mathrm{c}}^{\mathrm{e}}} \alpha_{\mathrm{c}}^{\mathrm{e}} \llbracket \boldsymbol{n} \cdot\left(\boldsymbol{\Lambda}_{h}^{\boldsymbol{T}^{0}}: \boldsymbol{\epsilon}_{\boldsymbol{E}}^{k-1}\right) \rrbracket \cdot \llbracket \boldsymbol{n} \cdot\left(\boldsymbol{\Lambda}_{h}^{\left.\boldsymbol{T}^{0}: \boldsymbol{\epsilon}_{\boldsymbol{E}}^{k}\right) \rrbracket \mathrm{d} \Sigma}\right. \\
& +\sum_{\Sigma_{\mathrm{c}}^{\mathrm{e}}} \int_{\Sigma_{\mathrm{c}}^{\mathrm{e}}}\left\{\left\{\boldsymbol{\epsilon}_{\boldsymbol{v}}^{k}-\boldsymbol{\epsilon}_{\boldsymbol{v}}^{k-1}\right\}\right\} \llbracket \boldsymbol{n} \cdot\left(\boldsymbol{\Lambda}_{h}^{\boldsymbol{T}^{0}}: \boldsymbol{\epsilon}_{\boldsymbol{E}}^{k}\right) \rrbracket \mathrm{d} \Sigma+\sum_{\Sigma_{\mathrm{c}}^{\mathrm{e}}} \int_{\Sigma_{\mathrm{c}}^{\mathrm{e}}} \llbracket \boldsymbol{\epsilon}_{\boldsymbol{v}}^{k} \rrbracket\left\{\left\{\boldsymbol{n} \cdot\left(\boldsymbol{\Lambda}_{h}^{\boldsymbol{T}^{0}}:\left(\boldsymbol{\epsilon}_{\boldsymbol{E}}^{k}-\boldsymbol{\epsilon}_{\boldsymbol{E}}^{k-1}\right)\right)\right\}\right\} \mathrm{d} \Sigma
\end{aligned}
$$

Substituting (B.5) and (B.9) into (B.10), and after some algebraic deductions,

$$
\begin{aligned}
& \sum_{\Omega^{\mathrm{e}}}\left(\frac{1}{\Delta t}\left\|\boldsymbol{\epsilon}_{\boldsymbol{v}}^{k}\right\|_{L^{2}\left(\Omega^{\mathrm{e}} ; \rho_{h}^{0}\right)}^{2}+\Delta t \int_{\Omega^{\mathrm{e}}} \boldsymbol{\epsilon}_{\boldsymbol{v}}^{k} \cdot \boldsymbol{K}_{h} \cdot \boldsymbol{\epsilon}_{\boldsymbol{v}}^{k} \mathrm{~d} \Omega+\gamma^{\mathrm{e}}\left\|\boldsymbol{\epsilon}_{\boldsymbol{v}}^{k}\right\|_{L^{2}\left(\Omega^{\mathrm{e}}\right)}^{2}+\frac{1}{\Delta t}\left\|\boldsymbol{\epsilon}_{\boldsymbol{E}}^{k}\right\|_{L^{2}\left(\Omega^{\mathrm{e}} ; \boldsymbol{\Lambda}_{h}^{T^{0}}\right)}^{2}\right. \\
& +\frac{\gamma^{\mathrm{e}}}{2}\left\|\nabla \boldsymbol{\epsilon}_{\boldsymbol{v}}^{k}\right\|_{L^{2}\left(\Omega^{\mathrm{e}}\right)}^{2}+\frac{\gamma^{\mathrm{e}}}{2 \Delta t^{2}}\left\|\boldsymbol{\epsilon}_{\boldsymbol{E}}^{k}\right\|_{L^{2}\left(\Omega^{\mathrm{e}}\right)}^{2}-\frac{1}{2 \gamma^{\mathrm{e}}}\left\|\boldsymbol{\Lambda}_{h}^{\boldsymbol{T}^{0}}: \boldsymbol{\epsilon}_{\boldsymbol{E}}^{k}\right\|_{L^{2}\left(\Omega^{\mathrm{e}}\right)}^{2} \\
& \left.+\frac{\gamma^{\mathrm{e}}}{2}\left\|\boldsymbol{r}_{\mathrm{f}}^{\mathrm{e}}\left(\llbracket \boldsymbol{n} \cdot \boldsymbol{\epsilon}_{\boldsymbol{v}}^{k} \rrbracket\right)-\boldsymbol{r}_{1}^{\mathrm{e}}\left(\llbracket \boldsymbol{\epsilon}_{\boldsymbol{v}}^{k} \rrbracket\right)-\boldsymbol{r}_{2}^{\mathrm{e}}\left(\left\{\left\{\boldsymbol{\epsilon}_{\boldsymbol{v}}^{k}-\boldsymbol{\epsilon}_{\boldsymbol{v}}^{k-1}\right\}\right\}\right)+\alpha_{\mathrm{c}}^{\mathrm{e}} \boldsymbol{r}_{2}^{\mathrm{e}}\left(\llbracket \boldsymbol{n} \cdot\left(\boldsymbol{\Lambda}^{\boldsymbol{T}^{0}}: \boldsymbol{\epsilon}_{\boldsymbol{E}}^{k-1}\right) \rrbracket\right)+\frac{1}{\gamma^{\mathrm{e}}}\left(\boldsymbol{\Lambda}_{h}^{\boldsymbol{T}^{0}}: \boldsymbol{\epsilon}_{\boldsymbol{E}}^{k}\right)\right\|_{L^{2}\left(\Omega^{\mathrm{e}}\right)}^{2}\right) \\
& +\sum_{\Sigma_{\mathrm{f}}^{\mathrm{e}}} \alpha_{\mathrm{f}}^{\mathrm{e}}(\Delta t+1)\left\|\llbracket \boldsymbol{n} \cdot \boldsymbol{\epsilon}_{\boldsymbol{v}}^{k} \rrbracket\right\|_{L^{2}\left(\Sigma_{\mathrm{f}}^{\mathrm{e}}\right)}^{2}+\sum_{\Sigma_{\mathrm{f}}^{\mathrm{e}}} \int_{\Sigma_{\mathrm{f}}^{\mathrm{e}}}\left(\boldsymbol{\epsilon}_{\boldsymbol{\tau}_{\mathrm{f}}}^{k} \cdot \boldsymbol{\epsilon}_{\boldsymbol{s}}^{k}-\left\{\left\{\boldsymbol{\epsilon}_{\boldsymbol{\tau}_{2}}^{k}\right\}\right\} \cdot \boldsymbol{\epsilon}_{\boldsymbol{s}}^{k}-\llbracket \boldsymbol{\epsilon}_{\boldsymbol{\tau}_{2}}^{k} \rrbracket \cdot\left\{\left\{\boldsymbol{\epsilon}_{\boldsymbol{v}}^{k}\right\}\right\}\right) \mathrm{d} \Sigma \\
& =-\sum_{\Sigma_{\mathrm{c}}^{\mathrm{e}}} \int_{\Sigma_{\mathrm{c}}^{\mathrm{e}}} \alpha_{\mathrm{c}}^{\mathrm{e}} \llbracket \boldsymbol{\epsilon}_{\boldsymbol{v}}^{k-1} \rrbracket \cdot \llbracket \boldsymbol{\epsilon}_{\boldsymbol{v}}^{k} \rrbracket \mathrm{d} \Sigma-\sum_{\Omega^{\mathrm{e}}} \int_{\Omega^{\mathrm{e}}} \boldsymbol{r}_{1}^{\mathrm{e}}\left(\llbracket \boldsymbol{\epsilon}_{\boldsymbol{v}}^{k} \rrbracket\right):\left(\boldsymbol{\Lambda}_{h}^{\boldsymbol{T}^{0}}: \boldsymbol{\epsilon}_{\boldsymbol{E}}^{k-1}\right) \mathrm{d} \Omega \\
& +\sum_{\Omega^{\mathrm{e}}} \int_{\Omega^{\mathrm{e}}} \boldsymbol{r}_{\mathrm{f}}^{\mathrm{e}}\left(\llbracket \boldsymbol{n} \cdot \boldsymbol{\epsilon}_{\boldsymbol{v}}^{k} \rrbracket\right):\left(\boldsymbol{\Lambda}_{h}^{\boldsymbol{T}^{0}}: \boldsymbol{\epsilon}_{\boldsymbol{E}}^{k}\right) \mathrm{d} \Omega .
\end{aligned}
$$

Based on (2.18), (B.6) and (B.7), and using Young's inequality, then (B.11) yields

$$
\begin{aligned}
\sum_{\Omega^{\mathrm{e}}}( & \left(\frac{C_{\rho^{0}}}{\Delta t}-\Delta t C_{\Phi^{0}}+\gamma^{\mathrm{e}}\right)\left\|\boldsymbol{\epsilon}_{\boldsymbol{v}}^{k}\right\|_{L^{2}\left(\Omega^{\mathrm{e}}\right)}^{2}+\frac{\gamma^{\mathrm{e}}}{2}\left\|\nabla \boldsymbol{\epsilon}_{\boldsymbol{v}}^{k}\right\|_{L^{2}\left(\Omega^{\mathrm{e}}\right)}^{2}+\left(\frac{C_{\boldsymbol{\Lambda}}}{\Delta t}+\frac{\gamma^{\mathrm{e}}}{2 \Delta t^{2}}\right)\left\|\boldsymbol{\epsilon}_{\boldsymbol{E}}^{k}\right\|_{L^{2}\left(\Omega^{\mathrm{e}}\right)}^{2}-\frac{1}{2 \gamma^{\mathrm{e}}}\left\|\boldsymbol{\Lambda}_{h}^{\boldsymbol{T}^{0}}: \boldsymbol{\epsilon}_{\boldsymbol{E}}^{k}\right\|_{L^{2}\left(\Omega^{\mathrm{e}}\right)}^{2} \\
& +\frac{\gamma^{\mathrm{e}}}{2} \| \boldsymbol{r}_{\mathrm{f}}^{\mathrm{e}}\left(\llbracket \boldsymbol{n} \cdot \boldsymbol{\epsilon}_{\boldsymbol{v}}^{k} \rrbracket\right)-\boldsymbol{r}_{1}^{\mathrm{e}}\left(\llbracket \boldsymbol{\epsilon}_{\boldsymbol{v}}^{k} \rrbracket\right)-\boldsymbol{r}_{2}^{\mathrm{e}}\left(\left\{\left\{\boldsymbol{\epsilon}_{\boldsymbol{v}}^{k}-\boldsymbol{\epsilon}_{\boldsymbol{v}}^{k-1}\right\}\right\}\right)+\alpha_{\mathrm{c}}^{\mathrm{e}} \boldsymbol{r}_{2}^{\mathrm{e}}\left(\llbracket \boldsymbol{n} \cdot\left(\boldsymbol{\Lambda}^{\boldsymbol{T}^{0}}: \boldsymbol{\epsilon}_{\boldsymbol{E}}^{k-1}\right) \rrbracket\right)+\frac{1}{\left.\gamma^{\mathrm{e}}\left(\boldsymbol{\Lambda}_{h}^{\boldsymbol{T}^{0}}: \boldsymbol{\epsilon}_{\boldsymbol{E}}^{k}\right) \|_{L^{2}\left(\Omega^{\mathrm{e}}\right)}^{2}\right)} \\
& +\sum_{\Sigma_{\mathrm{f}}^{\mathrm{e}}} \alpha_{\mathrm{f}}^{\mathrm{e}}(\Delta t+1)\left\|\llbracket \boldsymbol{n} \cdot \boldsymbol{\epsilon}_{\boldsymbol{v}}^{k} \rrbracket\right\|_{L^{2}\left(\Sigma_{\mathrm{f}}^{\mathrm{e}}\right)}^{2}+\sum_{\Sigma_{\mathrm{f}}^{\mathrm{e}}} \int_{\Sigma_{\mathrm{f}}^{\mathrm{e}}}\left(\boldsymbol{\epsilon}_{\boldsymbol{\tau}_{\mathrm{f}}}^{k} \cdot \boldsymbol{\epsilon}_{\boldsymbol{s}}^{k}-\left\{\left\{\boldsymbol{\epsilon}_{\boldsymbol{\tau}_{2}}^{k}\right\}\right\} \cdot \boldsymbol{\epsilon}_{\boldsymbol{s}}^{k}-\llbracket \boldsymbol{\epsilon}_{\boldsymbol{\tau}_{2}}^{k} \rrbracket \cdot\left\{\left\{\boldsymbol{\epsilon}_{\boldsymbol{v}}^{k}\right\}\right\}\right) \mathrm{d} \Sigma \\
\leq & \sum_{\Omega^{\mathrm{e}}} 4 C_{p} h^{-1}\left(\frac{\alpha_{\mathrm{c}}^{\mathrm{e}}}{2 \delta_{4}}\left\|\boldsymbol{\epsilon}_{\boldsymbol{v}}^{k-1}\right\|_{L^{2}\left(\Omega^{\mathrm{e}}\right)}^{2}+\frac{\alpha_{\mathrm{c}}^{\mathrm{e}} \delta_{4}+C_{r}^{\star} \delta_{5}}{2}\left\|\boldsymbol{\epsilon}_{\boldsymbol{v}}^{k}\right\|_{L^{2}\left(\Omega^{\mathrm{e}}\right)}^{2}\right)+\sum_{\Sigma_{\mathrm{f}}^{\mathrm{e}}} \frac{C_{r}^{\star}}{2 \delta_{6} h}\left\|\llbracket \boldsymbol{n} \cdot \boldsymbol{\epsilon}_{\boldsymbol{v}}^{k} \rrbracket\right\|_{L^{2}\left(\Sigma_{\mathrm{f}}^{\mathrm{e}}\right)}^{2} \\
& +\sum_{\Omega^{\mathrm{e}}}\left(\frac{1}{2 \delta_{5} h}\left\|\boldsymbol{\Lambda}_{h}^{\boldsymbol{T}^{0}}: \boldsymbol{\epsilon}_{\boldsymbol{E}}^{k-1}\right\|_{L^{2}\left(\Omega^{\mathrm{e}}\right)}^{2}+\frac{\delta_{6}}{2}\left\|\boldsymbol{\Lambda}_{h}^{\boldsymbol{T}^{0}}: \boldsymbol{\epsilon}_{\boldsymbol{E}}^{k}\right\|_{L^{2}\left(\Omega^{\mathrm{e}}\right)}^{2}\right)
\end{aligned}
$$


We also subtract (3.4c) from (4.1c) at step $k$, and let $\varphi=\epsilon_{\psi}^{k}$, such that

$$
\frac{1}{\Delta t}\left\|\epsilon_{\psi}^{k}\right\|_{L^{2}\left(\Sigma_{\mathrm{f}}^{\mathrm{e}}\right)}^{2}=-\int_{\Sigma_{\mathrm{f}}^{e}} \epsilon_{\mathcal{G}}^{k} \epsilon_{\psi}^{k} \mathrm{~d} \Sigma .
$$

Following the same procedure as $[66$, Section 6$]$, we get

$$
\begin{aligned}
& -\int_{\Omega^{\mathrm{e}}} \boldsymbol{\epsilon}_{\boldsymbol{\tau}_{\mathrm{f}}}^{k} \cdot \boldsymbol{\epsilon}_{\boldsymbol{s}}^{k} \mathrm{~d} \Omega \leq-C_{\mathcal{F}, s}\left\|\epsilon_{s}^{k}\right\|_{L^{2}\left(\Sigma_{\mathrm{f}}^{\mathrm{e}}\right)}^{2}+C_{\mathcal{F}, \sigma}^{\star} \Delta t\left(C_{I}+C_{I}^{\prime}\right)\left\|\epsilon_{\boldsymbol{v}}^{k}\right\|_{H^{1}\left(\Omega^{\mathrm{e}}\right)}^{2}+C_{\mathcal{F}, \psi}^{\star}\left(\frac{1}{2 \delta_{7}}\left\|\epsilon_{\psi}^{k}\right\|_{L^{2}\left(\Sigma_{\mathrm{f}}^{\mathrm{e}}\right)}^{2}+\frac{\delta_{7}}{2}\left\|\epsilon_{s}^{k}\right\|_{L^{2}\left(\Sigma_{\mathrm{f}}^{\mathrm{e}}\right)}^{2}\right), \\
& \int_{\Omega^{\mathrm{e}}}\left(\left\{\left\{\boldsymbol{\epsilon}_{\tau_{2}}^{k}\right\}\right\} \cdot \epsilon_{\boldsymbol{s}}^{k}+\llbracket \epsilon_{\boldsymbol{\tau}_{2}}^{k} \rrbracket \cdot\left\{\left\{\boldsymbol{\epsilon}_{\boldsymbol{v}}^{k}\right\}\right\}\right) \mathrm{d} \Omega \leq \Delta t C_{I}^{\prime}\left\|\boldsymbol{\epsilon}_{\boldsymbol{v}}^{k}\right\|_{H^{1}\left(\Omega^{\mathrm{e}}\right)}^{2}, \\
& -\int_{\Sigma_{\mathrm{f}}^{\mathrm{e}}} \epsilon_{\mathcal{G}}^{k} \epsilon_{\psi}^{k} \mathrm{~d} \Sigma \leq C_{\mathcal{G}, s}^{\star}\left(\frac{1}{2 \delta_{8}}\left\|\epsilon_{\psi}^{k}\right\|_{L^{2}\left(\Sigma_{\mathrm{f}}^{\mathrm{e}}\right)}^{2}+\frac{\delta_{8}}{2}\left\|\epsilon_{s}^{k}\right\|_{L^{2}\left(\Sigma_{\mathrm{f}}^{\mathrm{e}}\right)}^{2}\right)-C_{\mathcal{G}, \psi}\left\|\epsilon_{\psi}^{k}\right\|_{L^{2}\left(\Sigma_{\mathrm{f}}^{\mathrm{e}}\right)}^{2} .
\end{aligned}
$$

We let $\delta_{4}=\delta_{5}=1, \delta_{6}=\frac{C_{r}^{\star}}{2 \alpha_{\mathrm{f}}^{\mathrm{e}}(\Delta t+1) h}$, and $C_{\mathcal{F}, \psi}^{\star} \delta_{7}=C_{\mathcal{G}, s}^{\star} \delta_{8}=C_{\mathcal{F}, s}$, and substitute (B.13) and (B.14) into (B.12), such that

$$
\begin{aligned}
& \sum_{\Omega^{\mathrm{e}}}\left(\quad\left(\frac{C_{\rho^{0}}}{\Delta t}-\Delta t C_{\phi^{0}}+\gamma^{\mathrm{e}}-\Delta t\left(C_{\mathcal{F}, \sigma}^{\star}\left(C_{I}+C_{I}^{\prime}\right)+C_{I}^{\prime}\right)-\frac{2 C_{p} \alpha_{\mathrm{c}}^{\mathrm{e}}+2 C_{p} C_{r}^{\star}}{h}\right)\left\|\epsilon_{\boldsymbol{v}}^{k}\right\|_{L^{2}\left(\Omega^{\mathrm{e}}\right)}^{2}\right. \\
& \quad+\left(\frac{\gamma^{\mathrm{e}}}{2}-\Delta t\left(C_{\mathcal{F}, \sigma}^{\star}\left(C_{I}+C_{I}^{\prime}\right)+C_{I}^{\prime}\right)\right)\left\|\nabla \epsilon_{\boldsymbol{v}}^{k}\right\|_{L^{2}\left(\Omega^{\mathrm{e}}\right)}^{2}+\left(\frac{C_{\boldsymbol{\Lambda}}}{\Delta t}+\frac{\gamma^{\mathrm{e}}}{2 \Delta t^{2}}-\left(C_{\boldsymbol{\Lambda}}^{\star}\right)^{2}\left(\frac{1}{2 \gamma^{\mathrm{e}}}+\frac{C_{r}^{\star}}{4 \alpha_{\mathrm{f}}^{\mathrm{e}}(\Delta t+1) h}\right)\right)\left\|\epsilon_{\boldsymbol{E}}^{k}\right\|_{L^{2}\left(\Omega^{\mathrm{e}}\right)}^{2} \\
& \quad+\sum_{\Sigma_{\mathrm{f}}^{\mathrm{e}}}\left(\frac{1}{\Delta t}+C_{\mathcal{G}, \psi}-\frac{C_{\mathcal{F}, \psi}^{\star 2}}{2 C_{\mathcal{F}, s}}-\frac{C_{\mathcal{G}, s}^{\star 2}}{2 C_{\mathcal{F}, s}}\right)\left\|\epsilon_{\psi}^{k}\right\|_{L^{2}\left(\Sigma_{\mathrm{f}}^{\mathrm{e}}\right)^{2}}^{2} \\
& \leq \sum_{\Omega^{\mathrm{e}}}\left(\frac{2 C_{p} \alpha_{\mathrm{c}}^{\mathrm{e}}}{h}\left\|\epsilon_{\boldsymbol{v}}^{k-1}\right\|_{L^{2}\left(\Omega^{\mathrm{e}}\right)}^{2}+\frac{\left(C_{\boldsymbol{\Lambda}}^{\star}\right)^{2}}{2 h}\left\|\epsilon_{\boldsymbol{E}}^{k-1}\right\|_{L^{2}\left(\Omega^{\mathrm{e}}\right)}^{2}\right)
\end{aligned}
$$

Clearly, (B.15) is a contraction shown in (B.1) if the criteria described in (B.2) hold.

REMARK 3. Our proof above assumes a fixed single-rate time step $\Delta t$ and mesh size $h$, and the constants $C_{1}-C_{4}$ in Theorem B.1 depend on the initial gravitational potential, prestress tensor, elastic material parameters, fault geometry as well as $\Delta t$ and $h$. The value of $\gamma^{\mathrm{e}}$ can be chosen proportional to $\Delta t$ (given that $\Delta t<<1$ and is proportional to $h$ ), which can be sufficiently small to make the regularized, spatially and temporally discretized problem asymptotically approach the original one with pure elasticity. Moreover, $\gamma^{\mathrm{e}}$ can be assigned elementwise, as already mentioned in Remark 2. In particular, it only requires that $\gamma^{\mathrm{e}}$ takes strictly positive value within elements that are attached to the rupture surface.

Appendix C. The reduced problem of nonlinear friction with Newton's method.

In this appendix we obtain a matrix representation of the iterative scheme described by Problem 4. We consider a tetrahedral mesh with $K$ finite elements, and its interior surface mesh with $\widetilde{K_{\mathrm{f}}}$ triangular elements that represents the fault. We also assume that each tetrahedral element $\Omega^{\mathrm{e}}$ is connected to no more than one rupture facet $\Sigma_{\mathrm{f}}^{\mathrm{e}}$. The implementation of the numerical scheme takes four steps: first, we rewrite the discretized wave equations in Problem 4 into a matrix form; second, we use projection matrices $\left(\mathcal{P}^{\mathrm{e}}\right.$ and $\widetilde{\mathcal{P}}_{\mathrm{f}}^{\mathrm{e}}$ which are defined later) to reduce the problem to each rupture facet and its two adjacent tetrahedral elements; third, we conduct Gauss elimination and 
obtain the linear constraints between slip-velocity and friction force governed by the wave equation; finally, we obtain the matrix form of minimization problem (4.5), and write precisely the gradient and Hessian matrix.

Matrix form of discretized wave equation. We give the notation for unknown vectors corresponding with the unknown variables in Problem 4 below:

\begin{tabular}{l|ccccccc}
\hline variables on $\Omega$ or $\Sigma_{\mathrm{f}}$ & $\left(\boldsymbol{v}_{h}\right)_{j}$ & $\left(\boldsymbol{E}_{h}\right)_{i j}$ & $\left(\boldsymbol{u}_{h}\right)_{j}$ & $\left(\boldsymbol{s}_{h}\right)_{j}$ & $\left(\boldsymbol{\tau}_{\mathrm{f} h}\right)_{j}$ & $\sigma_{h}$ & $\psi_{h}$ \\
\hline local vectors on $\Omega^{\mathrm{e}}$ & $\mathcal{V}_{j}^{\mathrm{e}}$ & $\mathcal{E}_{i j}^{\mathrm{e}}$ & $\mathcal{U}_{j}^{\mathrm{e}}$ & & & & \\
\hline local vectors on $\Sigma^{\mathrm{e}}$ & $\widetilde{\mathcal{V}}_{j}^{\mathrm{e}}$ & & & $\widetilde{\mathcal{S}}_{j}^{\mathrm{e}}$ & $\widetilde{\mathcal{T}}_{j}^{\mathrm{e}}$ & $\tilde{\mathcal{N}}^{\mathrm{e}}$ & $\widetilde{\Psi}^{\mathrm{e}}$ \\
\hline global vectors & $\mathcal{V}_{j}$ & $\mathcal{E}_{i j}$ & $\mathcal{U}_{j}$ & & & & \\
\hline
\end{tabular}

We use the notation " ?" to represent quantities on the surface elements $\Sigma^{\mathrm{e}}$. The local vectors on each element $\Omega^{\mathrm{e}}$ are obtained by the expansion based on 3-D Lagrange polynomials $\left\{\varphi_{n}^{\mathrm{e}}(\boldsymbol{x})\right\}_{n=1}^{N_{p}}$ supported on $\Omega^{\mathrm{e}}$, for example,

$$
\left.\boldsymbol{v}_{j}(\boldsymbol{x})\right|_{\boldsymbol{x} \in \Omega^{\mathrm{e}}}=\sum_{n=1}^{N_{p}} v_{j n}^{\mathrm{e}} \varphi_{n}^{\mathrm{e}}(\boldsymbol{x}), \quad \mathcal{V}_{j}^{\mathrm{e}}:=\left[\left\{v_{j n}\right\}_{n=1}^{N_{p}}\right]^{\mathrm{T}},
$$

and likewise, the local vectors on $\Sigma^{\mathrm{e}}$ are obtained by the expansion based on 2-D Lagrange polynomials $\left\{\widetilde{\varphi}_{n}^{\mathrm{e}}(\boldsymbol{x})\right\}_{n=1}^{\widehat{N_{p}}}$ supported on $\Sigma^{\mathrm{e}}$, for example,

$$
\left.\boldsymbol{v}_{j}(\boldsymbol{x})\right|_{\boldsymbol{x} \in \Sigma^{\mathrm{e}}}=\sum_{n=1}^{\widetilde{N_{p}}} \widetilde{v}_{j n}^{\mathrm{e}} \widetilde{\varphi}_{n}^{\mathrm{e}}(\boldsymbol{x}), \quad \widetilde{\mathcal{V}}_{j}^{\mathrm{e}}:=\left[\left\{\widetilde{v}_{j n}\right\}_{n=1}^{\widetilde{N}_{p}}\right]^{\mathrm{T}} .
$$

We define the global mass matrix, $\mathcal{M}_{K} N_{p} \times K N_{p}$, whose diagonal blocks are local mass matrices with dimension $N_{p} \times N_{p}$, that is,

$$
\mathcal{M}_{m n}^{\mathrm{e}}:=\int_{\Omega^{\mathrm{e}}} \varphi_{m}^{\mathrm{e}} \varphi_{n}^{\mathrm{e}} \mathrm{d} \Omega, \quad m, n \in\left\{1,2, \cdots, N_{p}\right\}
$$

Similarly, the local surface mass matrices, $\widetilde{\mathcal{M}} \underset{\widetilde{N_{p}} \times \widetilde{N_{p}}}{\mathrm{e}}$, are defined by

$$
\widetilde{\mathcal{M}}_{m n}^{\mathrm{e}}:=\int_{\Sigma^{\mathrm{e}}} \widetilde{\varphi}_{m}^{\mathrm{e}} \widetilde{\varphi}_{n}^{\mathrm{e}} \mathrm{d} \Omega, \quad m, n \in\left\{1,2, \cdots, \widetilde{N}_{p}\right\}
$$

We introduce the block diagonal derivative matrices, $\mathcal{D}_{j}$, with the same dimension as $\mathcal{M}$. Their diagonal blocks $\mathcal{D}_{j}^{\mathrm{e}}$ are defined such that $\mathcal{D}_{j}^{\mathrm{e}} \mathcal{V}_{i}^{\mathrm{e}}$ yields the expansion

$$
\frac{\partial \boldsymbol{v}_{i}}{\partial \boldsymbol{x}_{j}}(\boldsymbol{x})=\sum_{\mathrm{e}} \sum_{n=1}^{N_{p}}\left(\mathcal{D}_{j}^{\mathrm{e}} \mathcal{V}_{i}^{\mathrm{e}}\right)_{n} \varphi_{n}^{\mathrm{e}}(\boldsymbol{x}), \quad \boldsymbol{x} \in \Omega .
$$

We then define the matrices $\mathcal{P}^{\mathrm{e}} N_{p} \times K N_{p}$ and $\widetilde{\mathcal{P}_{\mathrm{f}}^{\mathrm{e}}} \widetilde{N_{p}} \times \widetilde{K_{\mathrm{f}}} N_{p}$, whose entries take the value 0 or $1 . \mathcal{P}^{\mathrm{e}}$ projects global vectors to local vectors in each $\Omega^{\mathrm{e}}$ on the negative side of $\Sigma$ with regard to $\boldsymbol{n}$, such as $\mathcal{V}_{j}^{\mathrm{e}}=\mathcal{P}^{\mathrm{e}} \mathcal{V}_{j}$, and $\widetilde{\mathcal{P}_{\mathrm{f}}^{\mathrm{e}}}$ projects global vectors to local vectors on the negative side of $\Sigma_{\mathrm{f}}^{\mathrm{e}}$, such as $\widetilde{\mathcal{V}}_{j}^{\mathrm{e}}=\widetilde{\mathcal{P}_{\mathrm{f}}^{\mathrm{e}}} \mathcal{V}_{j}$. We employ the notation ".", which denotes quantities on the positive side of each $\Sigma^{\mathrm{e}}$. By the assumption that each tetrahedral element is connected to no more than one rupture facet, 
it is clear that for all matrices $\Xi$ that have the same block-diagonal structure as the global mass matrix $\mathcal{M}$ (for example, $\mathcal{D}_{j}$ and any diagonal matrices with dimension $K N_{p} \times K N_{p}$ ), the following holds true:

$$
\mathcal{P}^{\mathrm{e}} \Xi \underline{\widetilde{\mathcal{P}}^{\mathrm{e}}}=\underline{\mathcal{P}^{\mathrm{e}} \Xi} \widetilde{\mathcal{P}}_{\mathrm{f}}^{\mathrm{e}}=0
$$

A global vector and its corresponding local vectors in $\Omega^{\mathrm{e}}$ and $\Sigma_{\mathrm{f}}^{\mathrm{e}}$ satisfy

$$
\widetilde{\mathcal{V}}_{j}^{\mathrm{e}}=\widetilde{\mathcal{P}}_{\mathrm{f}}^{\mathrm{e}} \mathcal{V}_{j}=\widetilde{\mathcal{P}_{\mathrm{f}}^{\mathrm{e}}} \mathcal{P}^{\mathrm{e} \mathrm{T}} \mathcal{V}_{j}^{\mathrm{e}}, \quad \underline{\widetilde{\mathcal{V}}_{j}^{\mathrm{e}}}=\underline{\widetilde{\mathcal{P}_{\mathrm{f}}^{\mathrm{e}}}} \mathcal{V}_{j}=\underline{\widetilde{\mathcal{P}_{\mathrm{f}}^{\mathrm{e}}} \mathcal{P}^{\mathrm{eT}} \mathcal{V}_{j}^{\mathrm{e}}}
$$

We also define the matrix $\widetilde{\mathcal{P}_{\mathrm{c}}^{\mathrm{e}}}$ to represent the trace operation, which generates the regular numerial flux on continuous solid-solid interfaces. For example, $\frac{1}{2}\left(\widetilde{\mathcal{P}_{\mathrm{c}}^{\mathrm{e}}}+\underline{\mathcal{P}_{\mathrm{c}}^{\mathrm{e}}}\right) \mathcal{V}_{j}$ represents the central flux $\left\{\left\{\boldsymbol{v}_{j}\right\}\right\}$. We assume that the elastic parameters and prestress are piecewise constant, and define $\widetilde{\mathcal{Q}}_{i j}^{\mathrm{e}}$ that represents the additional surface term generated by the dynamically slipping boundary condition (see (2.13)), such that

$$
\widetilde{\mathcal{Q}}_{i j}^{\mathrm{e}} \mathcal{U}_{j}^{\mathrm{e}}=n_{m} \widetilde{\mathcal{P}_{\mathrm{f}}^{\mathrm{e}}} \mathcal{P}^{\mathrm{e} \mathrm{T}}\left(\mathcal{D}_{j}^{\mathrm{e}}-n_{j} n_{l} \mathcal{D}_{l}^{\mathrm{e}}\right) \mathcal{P}^{\mathrm{e}}\left(T_{m i}^{0} \mathcal{U}_{j}\right), \quad i, j, l, m \in\{1,2,3\}
$$

where $\boldsymbol{n}=\left[n_{1}, n_{2}, n_{3}\right]^{\mathrm{T}}$ is the normal vector of facet $\Sigma_{\mathrm{f}}^{\mathrm{e}}$. We let $\Gamma=\operatorname{diag}\left(\left\{\gamma^{\mathrm{e}}\right\}_{\Omega^{\mathrm{e}} \subset \Omega}\right)$ be a diagonal matrix with dimension $K N_{p} \times K N_{p}$. Below, we rewrite the equation of motion (4.1a) in matrix form, where $\mathcal{W}$ corresponds to the test function $\boldsymbol{w}_{h}$, (C.5)

$$
\begin{aligned}
& \frac{1}{\Delta t} \mathcal{W}_{i}^{\mathrm{T}} \mathcal{M} \rho^{0} \mathcal{V}_{i}^{(n, k)}+\mathcal{W}_{i}^{T} \mathcal{M} \Phi_{i j}^{0} \mathcal{U}_{j}^{(n, k)}+\mathcal{W}_{i}^{\mathrm{T}} \mathcal{M} \Gamma \mathcal{V}_{i}^{(n, k)}+\mathcal{W}_{i}^{\mathrm{T}} \mathcal{D}_{j}^{T} \mathcal{M} \Lambda_{j i l m}^{\boldsymbol{T}^{0}} \mathcal{E}_{l m}^{(n, k)}+\frac{1}{\Delta t} \mathcal{W}_{i}^{\mathrm{T}} \mathcal{D}_{j}^{T} \mathcal{M} \Gamma \mathcal{E}_{j i}^{(n, k)} \\
& +\mathcal{W}_{i}^{\mathrm{T}} \sum_{\Sigma_{\mathrm{f}}^{\mathrm{e}}}\left(\left(\underline{\widetilde{\widetilde{\mathcal{f}}_{\mathrm{f}}^{\mathrm{e}}}}-\widetilde{\widetilde{\mathcal{P}}_{\mathrm{f}}^{\mathrm{e}}}\right)^{\mathrm{T}} \widetilde{\mathcal{M}^{\mathrm{e}}}\left(\widetilde{\mathcal{T}}_{i}^{\mathrm{e}}-n_{i} \widetilde{\mathcal{N}}^{\mathrm{e}}\right)+\underline{\widetilde{\mathcal{P}}_{\mathrm{f}}^{\mathrm{e}}} \widetilde{\mathcal{M}}^{\mathrm{e}} \underline{\widetilde{\mathcal{Q}}_{i j}^{\mathrm{e}} \mathcal{U}_{j}^{\mathrm{e}(n, k)}}-\widetilde{\mathcal{P}}_{\mathrm{f}}^{\mathrm{e}} \widetilde{\mathcal{M}}^{\mathrm{e}} \widetilde{\mathcal{Q}}_{i j}^{\mathrm{e}} \mathcal{U}_{j}^{\mathrm{e}(n, k)}\right) \\
& +\mathcal{W}_{i}^{\mathrm{T}}\left(\sum_{\Sigma_{\mathrm{f}}^{\mathrm{e}}} \alpha_{\mathrm{f}}^{\mathrm{e}} n_{i} n_{j}\left(\underline{\widetilde{\widetilde{\mathcal{f}}_{\mathrm{f}}^{\mathrm{e}}}}-\widetilde{\widetilde{\mathcal{P}}_{\mathrm{f}}^{\mathrm{e}}}\right)^{\mathrm{T}} \widetilde{\mathcal{M}^{\mathrm{e}}}\left(\underline{\widetilde{\mathcal{P}_{\mathrm{f}}^{\mathrm{e}}}}-\widetilde{\mathcal{P}_{\mathrm{f}}^{\mathrm{e}}}\right)\right)\left(\mathcal{V}_{j}^{(n, k)}+\mathcal{U}_{j}^{(n, k)}\right) \\
& =\mathcal{W}_{i}^{\mathrm{T}}\left(\sum_{\Sigma_{\mathrm{f}}^{\mathrm{e}}} n_{j}\left(\underline{\widetilde{\mathcal{P}_{\mathrm{c}}^{\mathrm{e}}}}-\widetilde{\mathcal{P}_{\mathrm{c}}^{\mathrm{e}}}\right)^{\mathrm{T}} \widetilde{\mathcal{M}^{\mathrm{e}}} \widetilde{\mathcal{P}_{\mathrm{c}}^{\mathrm{e}}}\right)\left(T_{i j}^{0}+T_{i j}^{\delta(t)}\right)+\frac{1}{\Delta t}\left(\mathcal{W}_{i}^{\mathrm{T}} \mathcal{M} \rho^{0} \mathcal{V}_{i}^{(n-1)}+\mathcal{W}_{i}^{\mathrm{T}} \mathcal{D}_{j}^{T} \mathcal{M} \Gamma \mathcal{E}_{j i}^{(n-1)}\right) \\
& -\frac{1}{2} \mathcal{W}_{i}^{\mathrm{T}}\left(\sum_{\Sigma_{\mathrm{c}}^{\mathrm{e}}} n_{j}\left(\underline{\widetilde{\mathcal{P}_{\mathrm{c}}^{\mathrm{e}}}}-\widetilde{\mathcal{P}_{\mathrm{c}}^{\mathrm{e}}}\right)^{\mathrm{T}} \widetilde{\mathcal{M}^{\mathrm{e}}}\left(\underline{\widetilde{\mathcal{P}_{\mathrm{c}}^{\mathrm{e}}}}+\widetilde{\mathcal{P}_{\mathrm{c}}^{\mathrm{e}}}\right)\right) \Lambda_{j i l m}^{T^{0}} \mathcal{E}_{l m}^{(n, k-1)} \\
& -\mathcal{W}_{i}^{\mathrm{T}}\left(\sum_{\Sigma_{\mathrm{c}}^{\mathrm{e}}} \alpha_{\mathrm{c}}^{\mathrm{e}}\left(\underline{\widetilde{\mathcal{P}_{\mathrm{c}}^{\mathrm{e}}}}-\widetilde{\mathcal{P}_{\mathrm{c}}^{\mathrm{e}}}\right)^{\mathrm{T}} \widetilde{\mathcal{M}^{\mathrm{e}}}\left(\underline{\widetilde{\mathcal{P}_{\mathrm{c}}^{\mathrm{e}}}}-\widetilde{\mathcal{P}_{\mathrm{c}}^{\mathrm{e}}}\right)\right) \mathcal{V}_{i}^{(n, k-1)} \\
& :=\frac{1}{\Delta t} \mathcal{W}_{i}^{\mathrm{T}} \mathcal{M} \mathfrak{V}_{i}^{(n, k)}
\end{aligned}
$$

In the above, all terms containing $1 / \Delta t$ corresponds to the time derivative ones in (3.2a). We also highlight that all the terms regarding the source term, the variables from the previous time step (denoted by "(. $)^{(n-1)}$ ") and those from the previous iteration of current time step (denoted by "(. $)^{(n, k-1) ")}$ are moved to the right-hand-side and involved in a simplified notation, $\mathfrak{V}_{i}^{(n, k)}$. We also rewrite the strain-velocity relation, (4.1b), in a similar manner, using test function $\mathcal{H}$ corresponding 
to $\boldsymbol{H}_{h}$,

$$
\begin{aligned}
& \frac{1}{\Delta t} \mathcal{H}_{i j}^{\mathrm{T}} \mathcal{M} \mathcal{E}_{i j}^{(n, k)}+\mathcal{H}_{i j}^{\mathrm{T}} \mathcal{D}_{i}^{T} \mathcal{M} \mathcal{V}_{j}^{(n, k)}+\frac{1}{2} \mathcal{H}_{i j}^{\mathrm{T}}\left(\sum_{\Sigma_{\mathrm{f}}^{\mathrm{e}}} n_{i}\left(\underline{\widetilde{\mathcal{P}}}-\widetilde{\mathcal{P}^{\mathrm{e}}}\right)^{\mathrm{T}} \widetilde{\mathcal{M}^{\mathrm{e}}}\left(\underline{\widetilde{\mathcal{P}}}+\widetilde{\mathcal{P}^{\mathrm{e}}}\right)\right) \mathcal{V}_{j}^{(n, k)} \\
& +\frac{1}{2} \mathcal{H}_{i j}^{\mathrm{T}}\left(\sum_{\Sigma_{\mathrm{f}}^{\mathrm{e}}} n_{i}(\widetilde{\widetilde{\mathcal{P}}}+\widetilde{\mathcal{P} \mathrm{e}})^{\mathrm{T}} \widetilde{\mathcal{M}}^{\mathrm{e}} \widetilde{\mathcal{S}}_{j}^{\mathrm{e}}(n, k)\right) \\
& =\frac{1}{\Delta t} \mathcal{H}_{i j}^{\mathrm{T}} \mathcal{M E}_{i j}^{(n-1)}-\frac{1}{2} \mathcal{H}_{i j}^{\mathrm{T}}\left(\sum_{\Sigma_{\mathrm{c}}^{\mathrm{e}}} n_{i}\left(\underline{\widetilde{\mathcal{P}}}-\widetilde{\mathcal{P}^{\mathrm{e}}}\right)^{\mathrm{T}} \widetilde{\mathcal{M}^{\mathrm{e}}}\left(\underline{\mathcal{P}^{\mathrm{e}}}+\widetilde{\mathcal{P}^{\mathrm{e}}}\right)\right) \mathcal{V}_{j}^{(n, k-1)} \\
& -\mathcal{H}_{i j}^{\mathrm{T}}\left(\sum_{\Sigma_{\mathrm{c}}^{\mathrm{e}}} \alpha_{\mathrm{c}}^{\mathrm{e}} n_{i} n_{p}(\widetilde{\mathcal{P} \mathrm{e}}-\widetilde{\mathcal{P}})^{\mathrm{T}} \widetilde{\mathcal{M}^{\mathrm{e}}}(\widetilde{\mathcal{P} \mathrm{e}}-\widetilde{\mathcal{P}})\right) \Lambda_{p j l m}^{T^{0}} \mathcal{E}_{l m}^{(n, k-1)} \\
& :=\frac{1}{\Delta t} \mathcal{H}_{i j}^{\mathrm{T}} \mathcal{M} \mathfrak{E}_{i j}^{(n, k)} \text {, }
\end{aligned}
$$

and the right-hand-side involving "(. $)^{(n-1) "}$ and "(. $)^{(n, k-1)}$ " terms are involved in the simplified notation $\mathfrak{E}_{i j}^{(n, k)}$.

Projection onto rupture surface. Based on (C.5) and (C.6), the solid-solid inter-connections between elements are involved in the right-hand-sides, which can be computed using a standard time-domain DG scheme. The left-hand-sides, which are part of the implicit nonlinear problem, only involve local matrix-vector production within each element and interactions across rupture facets. To localize the scheme to each rupture facet and its adjacent pair of elements, we let $\mathcal{W}_{i}^{\mathrm{T}} \mathcal{M}=\Delta t \mathcal{P}^{\mathrm{e}}$ and $\mathcal{H}_{i j}^{\mathrm{T}} \Lambda_{j i l m}^{\boldsymbol{T}^{0} \mathrm{e}} \mathcal{M}=\Delta t \mathcal{P}^{\mathrm{e}}$ in (C.5) and (C.6), and apply (C.4), which gives

$$
\begin{aligned}
& \left(\rho^{0 \mathrm{e}}+\Delta t \gamma^{\mathrm{e}}\right) \mathcal{V}_{i}^{\mathrm{e}(n, k)}+\Delta t \Phi_{i j}^{0 \mathrm{e}} \mathcal{U}_{j}^{\mathrm{e}(n, k)}+\overline{\mathcal{D}}_{j}^{\mathrm{e}}\left(\Delta t \Lambda_{j i l m}^{T^{0} \mathrm{e}}+\gamma^{\mathrm{e}} \delta_{j l} \delta_{i m}\right) \mathcal{E}_{l m}^{\mathrm{e}(n, k)}-\Delta t \mathcal{J}^{\mathrm{e}} \widetilde{\mathcal{T}}_{i}^{\mathrm{e}}+\Delta t n_{i} \mathcal{J}^{\mathrm{e}} \tilde{\mathcal{N}}^{\mathrm{e}} \\
& -\Delta t \mathcal{J}^{\mathrm{e}} \widetilde{\mathcal{Q}}_{i j}^{\mathrm{e}} \mathcal{U}_{j}^{\mathrm{e}(n, k)}-\Delta t \alpha_{\mathrm{f}}^{\mathrm{e}} n_{i} n_{j} \mathcal{J}^{\mathrm{e}}\left(\underline{\tilde{\mathcal{L}}^{\mathrm{e}}\left(\mathcal{V}_{j}^{\mathrm{e}(n, k)}+\mathcal{U}_{j}^{\mathrm{e}(n, k)}\right)}-\widetilde{\mathcal{L}}^{\mathrm{e}}\left(\mathcal{V}_{j}^{\mathrm{e}(n, k)}+\mathcal{U}_{j}^{\mathrm{e}(n, k)}\right)\right)=\mathfrak{V}_{i}^{\mathrm{e}(n, k)},
\end{aligned}
$$

$\mathcal{E}_{i j}^{\mathrm{e}(n, k)}+\Delta t \overline{\mathcal{D}}_{i}^{\mathrm{e}} \mathcal{V}_{j}^{\mathrm{e}(n, k)}-\frac{\Delta t}{2} n_{i} \mathcal{J}^{\mathrm{e}}\left(\underline{\widetilde{\mathcal{L}}}^{\mathrm{e}}{ }_{j}^{\mathrm{e}(n, k)}+\widetilde{\mathcal{L}}^{\mathrm{e}} \mathcal{V}_{j}^{\mathrm{e}(n, k)}-\widetilde{\mathcal{S}}_{j}^{\mathrm{e}(n, k)}\right)=\mathfrak{E}_{i j}^{\mathrm{e}(n, k)}$,

with

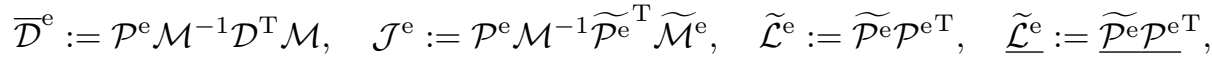

$$
\begin{aligned}
& \mathfrak{V}_{i}^{\mathrm{e}(n, k)}:=\mathcal{P}^{\mathrm{e}} \mathfrak{V}_{i}^{(n, k)}, \quad \text { and } \mathfrak{E}_{i j}^{\mathrm{e}(n, k)}:=\mathcal{P}^{\mathrm{e}} \mathfrak{E}_{i j}^{(n, k)}
\end{aligned}
$$

We get similar equations on the other side of the rupture surface by applying $\mathcal{W}_{i}^{\mathrm{T}} \mathcal{M}=\Delta t \underline{\mathcal{P}^{\mathrm{e}}}$ and 
$\mathcal{H}_{i j}^{\mathrm{T}} \Lambda_{j i l m}^{\boldsymbol{T}^{0} \mathrm{e}} \mathcal{M}=\Delta t \underline{\mathcal{P}^{\mathrm{e}}}$ to $(\mathrm{C} .5)$ and (C.6), such that

(C.8a)

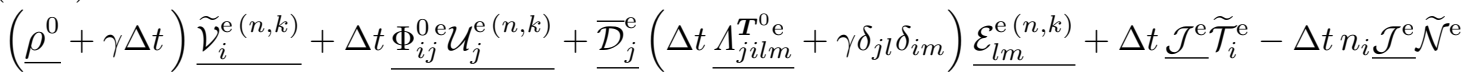

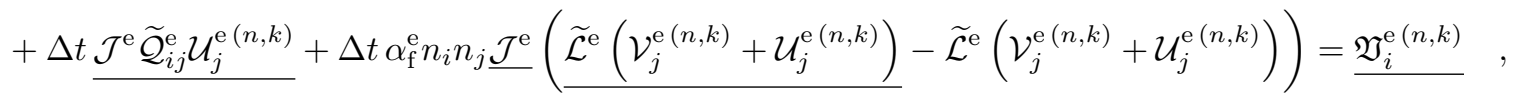

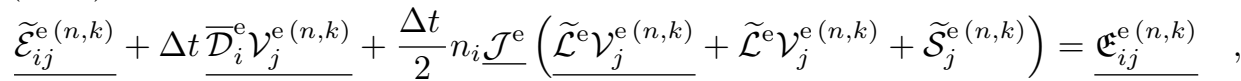

with

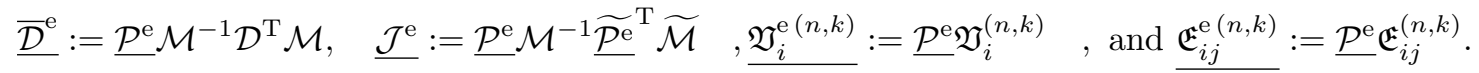

We also rewrite (4.2a), which contains only local operations, in terms of local vectors,

$$
\widetilde{\mathcal{N}}^{\mathrm{e}(n, k)}+\frac{n_{i} n_{j}}{2}\left(\underline{\widetilde{\mathcal{L}}^{\mathrm{e}} \Lambda_{i j l m}^{\boldsymbol{T}^{0} \mathrm{e}} \mathcal{E}_{l m}^{\mathrm{e}(n, k)}}+\widetilde{\mathcal{L}}^{\mathrm{e}} \Lambda_{i j l m}^{\boldsymbol{T}^{0} \mathrm{e}} \mathcal{E}_{l m}^{\mathrm{e}(n, k)}\right)-\frac{n_{i}}{2}\left(\underline{\widetilde{\mathcal{Q}}_{i j}^{\mathrm{e}} \mathcal{U}_{j}^{\mathrm{e}(n, k)}}+\widetilde{\mathcal{Q}}_{i j}^{\mathrm{e}} \mathcal{U}_{j}^{\mathrm{e}(n, k)}\right)=\widetilde{\mathfrak{N}}^{\mathrm{e}(n)},
$$

$$
\mathcal{U}_{i}^{\mathrm{e}(n, k)}-\Delta t \mathcal{V}_{i}^{\mathrm{e}(n, k)}=\mathcal{U}_{i}^{\mathrm{e}(n-1)}, \quad \underline{\mathcal{U}_{i}^{\mathrm{e}(n, k)}}-\Delta t \underline{\mathcal{V}_{i}^{\mathrm{e}(n, k)}}=\underline{\mathcal{U}_{i}^{\mathrm{e}(n-1)}},
$$

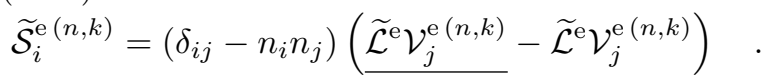

In $(\mathrm{C} .9 \mathrm{a}), \widetilde{\mathfrak{N}}^{\mathrm{e}(n)}:=-n_{i} n_{j} \widetilde{\mathcal{P}_{\mathrm{f}}^{\mathrm{e}}}\left(T_{i j}^{0}+T_{i j}^{\delta(n)}\right)$ is the backgound normal-stress applied to the rupture facet at time $t=n \Delta t$. We find that (C.7ab), (C.8ab) and (C.9a-c) form a system of equations with unknown vectors based on each rupture facet and the pair of its adjacent finite elements.

Linear constraint from wave equation. The minization problem (4.5) considers function of slip-velocity and friction force. Therefore we want to reduce $(\mathrm{C} .9 \mathrm{a}-\mathrm{c})$ to a simplified linear relation between these two specific variables. However, before we apply Gauss-elimination, we notice that the system $(\mathrm{C} .9 \mathrm{a}-\mathrm{c})$ is not full-rank, because $\widetilde{\mathcal{S}}_{i}^{\text {e }}$ and $\widetilde{\mathcal{T}}_{i}^{\text {e }}$ have vanishing normal components. We choose unit vectors $\boldsymbol{r}=\left[r_{1}, r_{2}, r_{3}\right]^{\mathrm{T}}$ and $\boldsymbol{t}=\left[t_{1}, t_{2}, t_{3}\right]^{\mathrm{T}}$ such that $[\boldsymbol{n}, \boldsymbol{r}, \boldsymbol{t}]$ forms an orthonormal matrix. We then denote by $\mathcal{K}=[\boldsymbol{r}, \boldsymbol{t}]^{\mathrm{T}}$ the matrix that projects vector quantities to the tangential plane. We find that (C.7ab), (C.8ab) and $(\mathrm{C} .9 \mathrm{a}-\mathrm{c})$ form the following linear system,

$$
\mathcal{A} \mathcal{Y}=\left[\begin{array}{c|c}
\mathcal{A}_{11} & \mathcal{A}_{12} \\
\hline \mathcal{A}_{21} & \mathcal{A}_{22}
\end{array}\right]\left[\begin{array}{l}
\mathcal{Y}_{1} \\
\hline \mathcal{Y}_{2}
\end{array}\right]=\left[\begin{array}{c}
\mathcal{Z}_{1} \\
\hline \mathcal{Z}_{2}
\end{array}\right]
$$

where

$$
\begin{aligned}
& \mathcal{Y}_{1}=\left[\begin{array}{llllll}
\mathcal{U} & \underline{\mathcal{U}} & \mathcal{E} & \underline{\mathcal{E}} & \mathcal{V} & \underline{\mathcal{V}}
\end{array}\right]^{\mathrm{T}}, \quad \mathcal{Y}_{2}=\left[\begin{array}{lll}
\widehat{\mathcal{S}} & \widetilde{\mathcal{N}} & \widehat{\mathcal{T}}
\end{array}\right]^{\mathrm{T}}, \\
& \mathcal{Z}_{1}=\left[\begin{array}{llllll}
\mathfrak{U} & \underline{\mathfrak{U}} & \mathfrak{E} & \underline{\mathfrak{E}} & \mathfrak{V} & \underline{\mathfrak{V}}
\end{array}\right]^{\mathrm{T}}, \quad \mathcal{Z}_{2}=\left[\begin{array}{ll}
\mathbf{0} & \tilde{\mathfrak{N}}
\end{array}\right]^{\mathrm{T}} .
\end{aligned}
$$


In the above, the vectors are defined as

$$
\begin{aligned}
\mathcal{U} & :=\left[\mathcal{U}_{1}^{\mathrm{e}(n, k)}, \mathcal{U}_{2}^{\mathrm{e}(n, k)}, \mathcal{U}_{3}^{\mathrm{e}(n, k)}\right]^{\mathrm{T}}, \quad \mathcal{V}:=\left[\mathcal{V}_{1}^{\mathrm{e}(n, k)}, \mathcal{V}_{2}^{\mathrm{e}(n, k)}, \mathcal{V}_{3}^{\mathrm{e}(n, k)}\right]^{\mathrm{T}}, \quad \widetilde{\mathcal{N}}:=\widetilde{\mathcal{N}}^{\mathrm{e}(n, k)}, \\
\mathcal{E} & :=\left[\mathcal{E}_{11}^{\mathrm{e}(n, k)}, \mathcal{E}_{21}^{\mathrm{e}(n, k)}, \mathcal{E}_{31}^{\mathrm{e}(n, k)}, \mathcal{E}_{12}^{\mathrm{e}(n, k)}, \mathcal{E}_{22}^{\mathrm{e}(n, k)}, \mathcal{E}_{32}^{\mathrm{e}(n, k)}, \mathcal{E}_{13}^{\mathrm{e}(n, k)}, \mathcal{E}_{23}^{\mathrm{e}(n, k)}, \mathcal{E}_{33}^{\mathrm{e}(n, k)}\right]^{\mathrm{T}}, \\
\widehat{\mathcal{S}} & :=\left[\widehat{\mathcal{S}}_{1}^{\mathrm{e}(n, k)}, \widehat{\mathcal{S}}_{2}^{\mathrm{e}(n, k)}\right]^{T}=\mathcal{K}\left[\widetilde{\mathcal{S}}_{1}^{\mathrm{e}(n, k)}, \widetilde{\mathcal{S}}_{2}^{\mathrm{e}(n, k)}, \widetilde{\mathcal{S}}_{3}^{\mathrm{e}(n, k)}\right]^{\mathrm{T}}, \\
\widehat{\mathcal{T}} & :=\left[\widehat{\mathcal{T}}_{1}^{\mathrm{e}(n, k)}, \widehat{\mathcal{T}}_{2}^{\mathrm{e}(n, k)}\right]^{T}=\mathcal{K}\left[\widetilde{\mathcal{T}}_{1}^{\mathrm{e}(n, k)}, \widetilde{\mathcal{T}}_{2}^{\mathrm{e}(n, k)}, \widetilde{\mathcal{T}}_{3}^{\mathrm{e}(n, k)}\right]^{\mathrm{T}}, \\
\mathfrak{U} & :=\left[\mathcal{U}_{1}^{\mathrm{e}(n-1)}, \mathcal{U}_{2}^{\mathrm{e}(n-1)}, \mathcal{U}_{3}^{\mathrm{e}(n-1)}\right]^{\mathrm{T}}, \quad \mathfrak{V}:=\left[\mathfrak{V}_{1}^{\mathrm{e}(n, k)}, \mathfrak{V}_{2}^{\mathrm{e}(n, k)}, \mathfrak{V}_{3}^{\mathrm{e}(n, k)}\right]^{\mathrm{T}}, \widetilde{\mathfrak{N}}=\widetilde{\mathfrak{N}}^{\mathrm{e}(n)}, \\
\mathfrak{E} & :=\left[\mathfrak{E}_{11}^{\mathrm{e}(n, k)}, \mathfrak{E}_{21}^{\mathrm{e}(n, k)}, \mathfrak{E}_{31}^{\mathrm{e}(n, k)}, \mathfrak{E}_{12}^{\mathrm{e}(n, k)}, \mathfrak{E}_{22}^{\mathrm{e}(n, k)}, \mathfrak{E}_{32}^{\mathrm{e}(n, k)}, \mathfrak{E}_{13}^{\mathrm{e}(n, k)}, \mathfrak{E}_{23}^{\mathrm{e}(n, k)}, \mathfrak{E}_{33}^{\mathrm{e}(n, k)}\right]^{\mathrm{T}} .
\end{aligned}
$$

We explicitly write matrix $\mathcal{A}$ as follows,

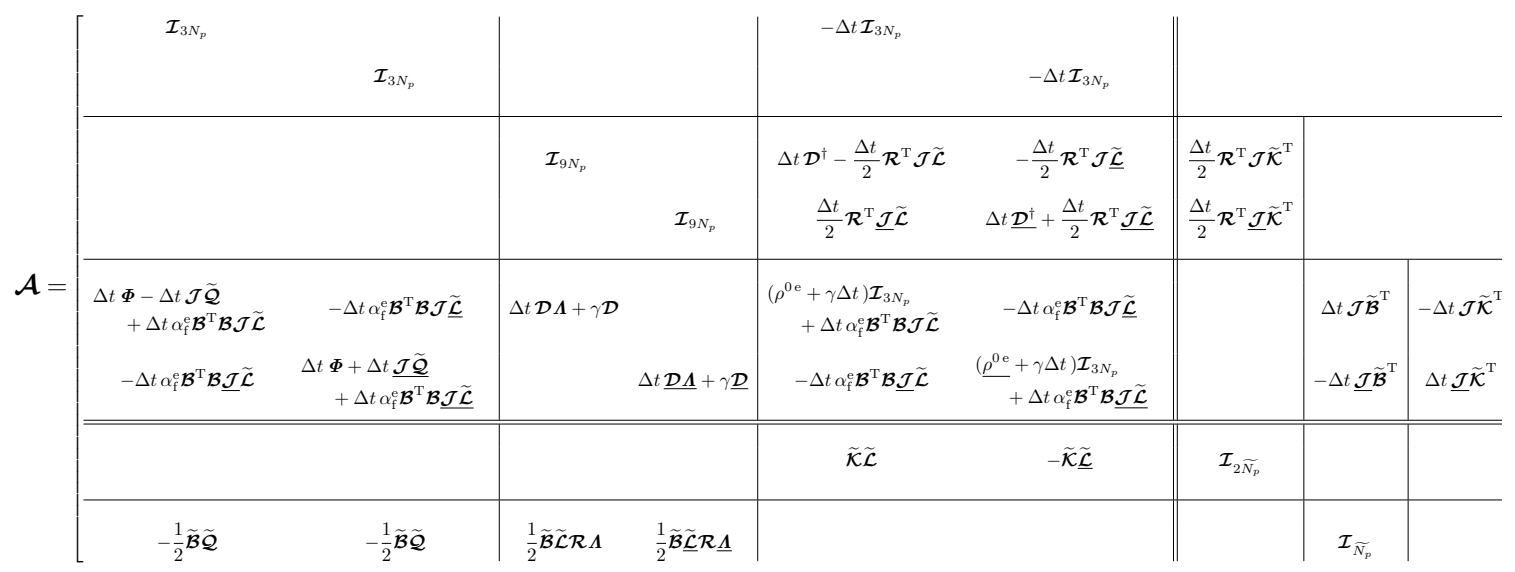

In the representation of matrix $\mathcal{A}$, the double lines divide blocks $\mathcal{A}_{i j}$ with $i, j \in\{1,2\}$, and as mentioned before, quantities with and without "." stand for the ones obtained from the "+" and "-" sides of the rupture facet, respectively. $\mathcal{I}_{N}$ stands for the $N \times N$ identity matrix, and the dense blocks $\mathcal{B}, \widetilde{\mathcal{B}}, \mathcal{R}, \widetilde{\mathcal{K}}, \mathcal{J}, \widetilde{\mathcal{L}}, \mathcal{D}, \mathcal{D}^{\dagger}$ and $\mathcal{Q}$ are given by

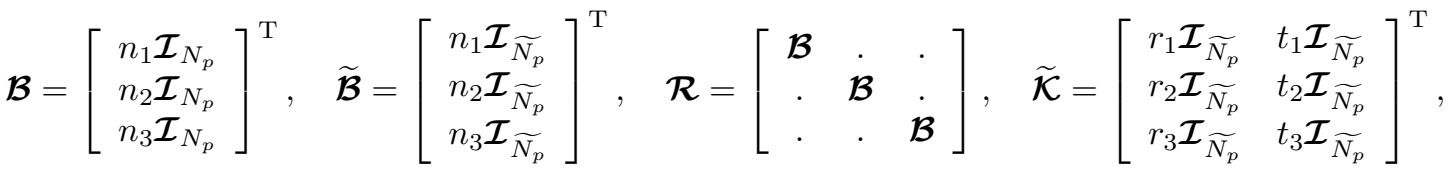

$$
\begin{aligned}
& \mathcal{J}=\left[\begin{array}{ccc}
\mathcal{J}^{\mathrm{e}} & \cdot & \cdot \\
\cdot & \mathcal{J}^{\mathrm{e}} & \cdot \\
\cdot & \cdot & \mathcal{J}^{\mathrm{e}}
\end{array}\right], \quad \widetilde{\mathcal{L}}=\left[\begin{array}{ccc}
\widetilde{\mathcal{L}}^{\mathrm{e}} & \dot{\dot{\mathcal{L}}^{\mathrm{e}}} & \cdot \\
\cdot & \dot{\widetilde{\mathcal{L}}^{\mathrm{e}}}
\end{array}\right] \\
& \mathcal{D}=\left[\begin{array}{ccc}
\mathcal{D} & \dot{1} & \cdot \\
\cdot & \mathcal{D} & \cdot \\
\cdot & \cdot & \mathcal{D}
\end{array}\right], \text { with } \mathcal{D}=\left[\begin{array}{lll}
\overline{\mathcal{D}}_{1}^{\mathrm{e}} & \overline{\mathcal{D}}_{2}^{\mathrm{e}} & \overline{\mathcal{D}}_{3}^{\mathrm{e}}
\end{array}\right] ; \quad \mathcal{D}^{\dagger}=\left[\begin{array}{ccc}
\mathcal{D}^{\dagger} & . & \cdot \\
\cdot & \mathcal{D}^{\dagger} & \cdot \\
\cdot & \cdot & \mathcal{D}^{\dagger}
\end{array}\right] \text {, with } \mathcal{D}^{\dagger}=\left[\begin{array}{c}
\overline{\mathcal{D}}_{1}^{\mathrm{e}} \\
\overline{\mathcal{D}_{2}^{\mathrm{e}}} \\
\overline{\mathcal{D}_{3}^{\mathrm{e}}}
\end{array}\right] ; \\
& (\mathcal{Q})_{i j}=\widetilde{\mathcal{Q}}_{i j}^{\mathrm{e}}, \quad i, j \in\{1,2,3\} .
\end{aligned}
$$

The $2 \times 2$ structure of $\mathcal{A}$ illustrates the composition of the coupled problem: $A_{11}$ represents the wave equation with time discretization, $A_{12}$ gives numerically the trace operation that maps particle 
velocity in the bulk volume to the slip velocity that resides on the rupture surface, $A_{21}$ considers the force equilibrium on the dynamically slipping boundary, and $A_{22}$ simply contains an identity matrix that brings friction force and normal stress perturbation into the system.

We conduct Gauss elimination on (C.10), which yields

$$
\left(\mathcal{A}_{22}-\mathcal{A}_{21} \mathcal{A}_{11}^{-1} \mathcal{A}_{12}\right) \mathcal{Y}_{2}=\mathcal{Z}_{2}-\mathcal{A}_{21} \mathcal{A}_{11}^{-1} \mathcal{Z}_{1}
$$

We let

$$
\mathcal{A}_{22}-\mathcal{A}_{21} \mathcal{A}_{11}^{-1} \mathcal{A}_{12}=: \overline{\mathcal{A}}=\left[\overline{\mathcal{A}}_{1} \mid \overline{\mathcal{A}}_{2}\right]
$$

with $\left(\overline{\mathcal{A}}_{1}\right)_{3 \widetilde{N_{p}} \times 2 \widetilde{N}_{p}}$ and $\left(\overline{\mathcal{A}}_{2}\right)_{3 \widetilde{N_{p}} \times 3 \widetilde{N}_{p}}$ submatrices of $\overline{\mathcal{A}}$, and let

$$
\overline{\overline{\mathcal{A}}}:=-\overline{\mathcal{A}}_{2}^{-1} \overline{\mathcal{A}}_{1}
$$

Then (C.11) becomes

$$
[\widetilde{\mathcal{N}}, \widehat{\mathcal{T}}]^{\mathrm{T}}=\overline{\overline{\mathcal{A}}} \widehat{\mathcal{S}}+\overline{\mathcal{A}}_{2}^{-1}\left(\mathcal{Z}_{2}-\mathcal{A}_{21} \mathcal{A}_{11}^{-1} \mathcal{Z}_{1}\right)
$$

Solution algorithm to the minimization problem. We take the derivative of (4.4ab), which yields

$$
\begin{aligned}
& \mathrm{d} \psi^{(n, k)}=\frac{\Delta t}{N} \sum_{i=1}^{N} \mathrm{~d} \theta^{i}, \quad \mathrm{~d} \theta^{i}=-\frac{\partial \mathcal{G}}{\partial s}\left(s^{(n), i}, \psi^{(n), i}\right) \frac{i}{N} \mathrm{~d} s-\frac{\partial \mathcal{G}}{\partial \psi}\left(s^{(n), i}, \psi^{(n), i}\right) \frac{\Delta t}{N} \sum_{j=1}^{i} \mathrm{~d} \theta^{j}, \\
& \text { with } s^{(n), i}=s^{(n-1)}+\frac{i}{N}\left(s^{(n, k)}-s^{(n-1)}\right), \quad \psi^{(n), i}=\psi^{(n-1)}+\frac{\Delta t}{N} \sum_{j=1}^{i} \theta^{j} .
\end{aligned}
$$

With a given nonlinear function $\mathcal{F}$ in $(2.10)$, we formulate a minimization problem as described in $(4.5)$,

$$
\widehat{\mathcal{S}}=\arg \min \mathfrak{L}(\widehat{\mathcal{S}}), \quad \text { with } \quad \mathfrak{L}=\frac{1}{2}\left\|\frac{\widehat{\mathcal{S}}}{|\widehat{\mathcal{S}}|} \mathcal{F}(\widetilde{\mathcal{N}}, \widehat{\mathcal{S}}, \widetilde{\Psi})-\widehat{\mathcal{T}}\right\|^{2}
$$

This minimization problem is constrained by (C.10) and (C.15). We obtain an explicit expression for the gradient,

$\mathfrak{G}_{j}:=\frac{\partial \mathfrak{L}}{\partial \widehat{\mathcal{S}}_{j}}=\sum_{l=1}^{2}\left(\frac{\widehat{\mathcal{S}}_{l}}{|\widehat{\mathcal{S}}|} \mathcal{F}-\widehat{\mathcal{T}}_{l}\right)\left(\delta_{l j} \frac{\mathcal{F}}{|\widehat{\mathcal{S}}|}+\frac{\widehat{\mathcal{S}}_{l} \widehat{\mathcal{S}}_{j}}{|\widehat{\mathcal{S}}|^{3}}\left(\left(\frac{\partial \mathcal{F}}{\partial s}+\frac{\partial \mathcal{F}}{\partial \psi} \frac{\mathrm{d} \psi}{\mathrm{d} s}\right)|\widehat{\mathcal{S}}|-\mathcal{F}\right)+\frac{\widehat{\mathcal{S}}_{l}}{|\widehat{\mathcal{S}}|} \frac{\partial \mathcal{F}}{\partial \sigma} \overline{\overline{\mathcal{A}}}_{0 j}-\overline{\overline{\mathcal{A}}}_{l j}\right)$,

and the Gauss-Newton Hessian

$$
\begin{aligned}
\mathfrak{H}_{i j}:=\frac{\partial^{2} \mathfrak{L}}{\partial \widehat{\mathcal{S}}_{i} \partial \widehat{\mathcal{S}}_{j}} \approx \sum_{l=1}^{2}( & \left(\delta_{l i} \frac{\mathcal{F}}{|\widehat{\mathcal{S}}|}+\frac{\widehat{\mathcal{S}}_{l} \widehat{\mathcal{S}}_{i}}{|\widehat{\mathcal{S}}|^{3}}\left(\left(\frac{\partial \mathcal{F}}{\partial s}+\frac{\partial \mathcal{F}}{\partial \psi} \frac{\mathrm{d} \psi}{\mathrm{d} s}\right)|\widehat{\mathcal{S}}|-\mathcal{F}\right)+\frac{\widehat{\mathcal{S}}}{|\widehat{\mathcal{S}}|} \frac{\partial \mathcal{F}}{\partial \sigma} \overline{\overline{\mathcal{A}}}_{0 i}-\overline{\overline{\mathcal{A}}}_{l i}\right)^{\mathrm{T}} . \\
& \left.\left(\delta_{l j} \frac{\mathcal{F}}{|\widehat{\mathcal{S}}|}+\frac{\widehat{\mathcal{S}}_{l} \widehat{\mathcal{S}}_{j}}{|\widehat{\mathcal{S}}|^{3}}\left(\left(\frac{\partial \mathcal{F}}{\partial s}+\frac{\partial \mathcal{F}}{\partial \psi} \frac{\mathrm{d} \psi}{\mathrm{d} s}\right)|\widehat{\mathcal{S}}|-\mathcal{F}\right)+\frac{\widehat{\mathcal{S}}}{|\widehat{\mathcal{S}}|} \frac{\partial \mathcal{F}}{\partial \sigma} \overline{\overline{\mathcal{A}}}_{0 j}-\overline{\overline{\mathcal{A}}}_{l j}\right)\right) .
\end{aligned}
$$

In the above, each $\overline{\overline{\mathcal{A}}}_{i j}(i \in\{0,1,2\}, j \in\{1,2\})$ is an $\widetilde{N}_{p} \times \widetilde{N}_{p}$ block of matrix $\overline{\overline{\mathcal{A}}}$, and $\frac{\mathrm{d} \psi}{\mathrm{d} s}$ is evaluated using (C.15).

We finally summarize the algorithm of solving the overall problem in Algorithm 1. 


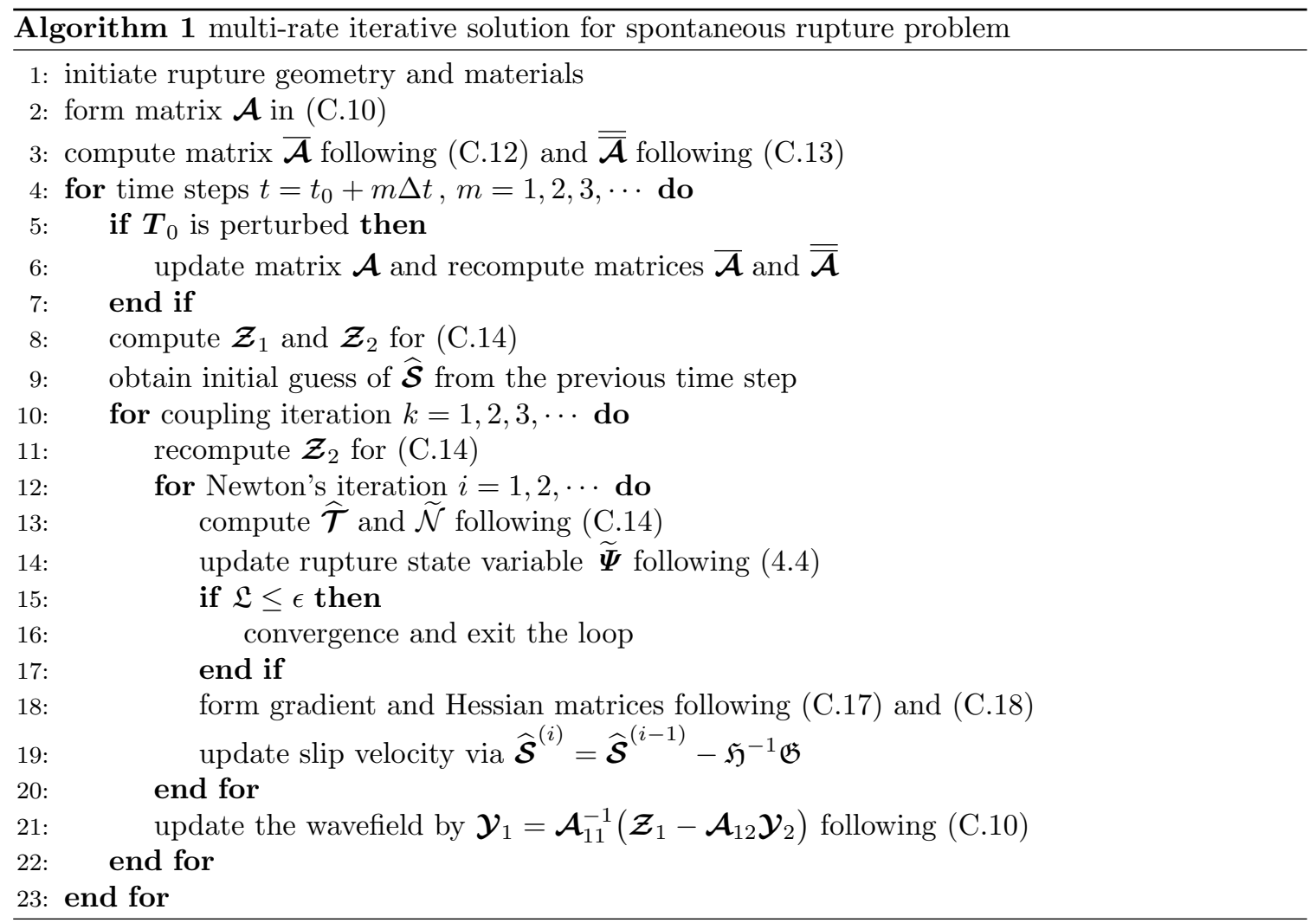

\section{REFERENCES}

[1] B. T. Aagandd, G. Anderson, and K. W. Hudnut, Dynamic rupture modeling of the transition from thrust to strike-slip motion in the 2002 Denali Fault Earthquake, Alaska, Bulletin of the Seismological Society of America, 94 (2004), pp. S190-S201.

[2] B. T. Aagaard, M. G. Knepley, and C. A. Williams, A domain decomposition approach to implementing fault slip in finite-element models of quasi-static and dynamic crustal deformation, Journal of Geophysical Research: Solid Earth, 118 (2013), pp. 3059-3079.

[3] T. Almani, K. Kumar, A. Dogru, G. Singh, and M. Wheeler, Convergence analysis of multirate fixed-stress split iterative schemes for coupling flow with geomechanics, Computer Methods in Applied Mechanics and Engineering, 311 (2016).

[4] J.-P. AMPUERO AND Y. Ben-Zion, Cracks, pulses and macroscopic asymmetry of dynamic rupture on a bimaterial interface with velocity-weakening friction, Geophysical Journal International, 173 (2008), pp. 674-692.

[5] J.-P. Ampuero And A. M. Rubin, Earthquake nucleation on rate and state faults-aging and slip laws, Journal of Geophysical Research: Solid Earth, 113 (2008).

[6] D. J. Andrews And Y. Ben-Zion, Wrinkle-like slip pulse on a fault between different materials, Journal of Geophysical Research: Solid Earth, 102 (1997), pp. 553-571.

[7] D. N. Arnold, F. Brezzi, B. Cockburn, and L. D. Marini, Unified analysis of discontinuous Galerkin methods for elliptic problems, SIAM journal on numerical analysis, 39 (2002), pp. 1749-1779.

[8] M. Benjemaa, N. Glinsky-Olivier, V. Cruz-Atienza, and J. Virieux, 3-D dynamic rupture simulations by a finite volume method, Geophysical Journal International, 178 (2009), pp. 541-560.

[9] J. Bogers, Transport in fractured media: well-posedness, upscaling and numerical schemes for problems involving a nonlinear transmission condition, master's thesis, Eindhoven University of Technology, 2011.

[10] K. Brazda, M. V. DE Hoop, And G. Hörmann, Variational formulation of the earth's elastic-gravitational deformations under low regularity conditions, Cambridge University Press, 2017, pp. 1-81.

[11] M. Campillo, P. Favreau, I. R. Ionescu, and C. Voisin, On the effective friction law of a heterogeneous fault, Journal of Geophysical Research: Solid Earth, 106 (2001), pp. 16307-16322.

[12] M. CAmpillo And I. R. Ionescu, Initiation of antiplane shear instability under slip dependent friction, Journal of Geophysical Research: Solid Earth, 102 (1997), pp. 20363-20371.

[13] A. Cochard And J. Rice, Fault rupture between dissimilar materials: ill-posedness, regularization, and slippulse response, Journal of Geophysical Research: Solid Earth, 105 (2000), pp. 25891-25907. 
[14] F. A. Dahlen and J. Tromp, Theoretical global seismology, Princeton university press, 1998.

[15] R. Davies, G. Foulger, A. Bindley, and P. Styles, Induced seismicity and hydraulic fracturing for the recovery of hydrocarbons, Marine and Petroleum Geology, 45 (2013), pp. 171-185.

[16] S. M. Day, L. A. Dalguer, N. Lapusta, and Y. Liu, Comparison of finite difference and boundary integral solutions to three-dimensional spontaneous rupture, Journal of Geophysical Research: Solid Earth, 110 (2005), pp. 1-23.

[17] J. D. De Basabe, M. K. Sen, And M. F. Wheeler, Elastic wave propagation in fractured media using the discontinuous Galerkin method, Geophysics, 81 (2016), pp. T163-T174.

[18] M. V. De Hoop, S. Holman, And H. Pham, On the system of elastic-gravitational equations describing the oscillations of the earth, arXiv preprint arXiv:1511.03200, (2015), pp. 1-51.

[19] J. De la Puente, J.-P. Ampuero, And M. Käser, Dynamic rupture modeling on unstructured meshes using a discontinuous Galerkin method, Journal of Geophysical Research: Solid Earth, 114 (2009).

[20] M. DumbSer And M. KäSER, An arbitrary high-order discontinuous Galerkin method for elastic waves on unstructured meshes-II. the three-dimensional isotropic case, Geophysical Journal International, 167 (2006), pp. 319-336.

[21] M. Dumbser, M. KÄser, And E. F. Toro, An arbitrary high-order discontinuous Galerkin method for elastic waves on unstructured meshes- $V$. local time stepping and p-adaptivity, Geophysical Journal International, 171 (2007), pp. 695-717.

[22] K. Duru And E. M. Dunham, Dynamic earthquake rupture simulations on nonplanar faults embedded in 3D geometrically complex, heterogeneous elastic solids, Journal of Computational Physics, 305 (2016), pp. 185207.

[23] A. M. Dziewonski and D. L. Anderson, Preliminary reference Earth model, Physics of the earth and planetary interiors, 25 (1981), pp. 297-356.

[24] P. Galvez, J.-P. Ampuero, L. Dalguer, S. Somala, and T. Nissen-Meyer, Dynamic earthquake rupture modelled with an unstructured 3-D spectral element method applied to the 2011 M9 Tohoku Earthquake, Geophysical Journal International, 198 (2014), pp. 1222-1240.

[25] P. H. Geubelle And J. R. Rice, A spectral method for three-dimensional elastodynamic fracture problems, Journal of the Mechanics and Physics of Solids, 43 (1995), pp. 1791-1824.

[26] M. J. Grote, M. Mehlin, and T. Mitkova, Runge-Kutta-based explicit local time-stepping methods for wave propagation, SIAM Journal on Scientific Computing, 37 (2015), pp. A747-A775.

[27] R. Harris, M. Barall, R. Archuleta, E. Dunham, B. Aagaard, J. Ampuero, H. Bhat, V. Cruz-Atienza, L. Dalguer, P. DAWson, ET AL., The SCEC/USGS dynamic earthquake rupture code verification exercise, Seismological Research Letters, 80 (2009), pp. 119-126.

[28] H. Huang And F. Costanzo, On the use of space-time finite elements in the solution of elasto-dynamic fracture problems, International Journal of Fracture, 127 (2004), pp. 119-146.

[29] I. R. Ionescu, Q.-L. NGUyen, AND S. Wolf, Slip-dependent friction in dynamic elasticity, Nonlinear Analysis: Theory, Methods \& Applications, 53 (2003), pp. 375-390.

[30] N. Kame, J. R. Rice, AND R. Dmowska, Effects of prestress state and rupture velocity on dynamic fault branching, Journal of Geophysical Research: Solid Earth, 108 (2003).

[31] Y. KANEKO AND N. LAPUSTA, Variability of earthquake nucleation in continuum models of rate-and-state faults and implications for aftershock rates, Journal of Geophysical Research: Solid Earth, 113 (2008).

[32] Y. Kaneko, N. Lapusta, And J.-P. Ampuero, Spectral element modeling of spontaneous earthquake rupture on rate and state faults: effect of velocity-strengthening friction at shallow depths, Journal of Geophysical Research: Solid Earth, 113 (2008), pp. 1-17.

[33] J. E. Kozdon, E. M. Dunham, And J. Nordström, Interaction of waves with frictional interfaces using summation-by-parts difference operators: weak enforcement of nonlinear boundary conditions, Journal of Scientific Computing, 50 (2012), pp. 341-367.

[34] S. Latour, M. Campillo, C. Voisin, I. Ionescu, J. Schmedes, and D. Lavallée, Effective friction law for small-scale fault heterogeneity in 3D dynamic rupture, Journal of Geophysical Research: Solid Earth, 116 (2011).

[35] J. C. Lozos, R. A. Harris, J. R. Murray, and J. J. Lienkaemper, Dynamic rupture models of earthquakes on the Bartlett Springs Fault, Northern California, Geophysical Research Letters, 42 (2015), pp. 4343-4349.

[36] S. Ma, R. J. Archuleta, And M. T. PAGe, Effects of large-scale surface topography on ground motions, as demonstrated by a study of the San Gabriel Mountains, Los Angeles, California, Bulletin of the Seismological Society of America, 97 (2007), pp. 2066-2079.

[37] X. Ma, S. Hajarolasvadi, G. Albertini, D. S. Kammer, and A. E. Elbanna, A hybrid finite element-spectral boundary integral approach: applications to dynamic rupture modeling in unbounded domains, International Journal for Numerical and Analytical Methods in Geomechanics, 43 (2019), pp. 317-338.

[38] R. Madariaga, J. Ampuero, and M. Adda-Bedia, Seismic radiation from simple models of earthquakes, Earthquakes: radiated Energy and the Physics of Faulting, (2006), pp. 223-236.

[39] J. A. A. C. MARTins AND J. T. Oden, Existence and uniqueness results for dynamic contact problems with nonlinear normal and friction interface laws, Nonlinear Analysis: Theory, Methods \& Applications, 11 (1987), pp. 407-428.

[40] P. Moczo, J. Kristek, And M. GÁLis, The finite-difference modelling of earthquake motions: Waves and ruptures, Cambridge University Press, 2014. 
[41] M. Möllhoff And C. J. Bean, Validation of elastic wave measurements of rock fracture compliance using numerical discrete particle simulations, Geophysical Prospecting, 57 (2009), pp. 883-895.

[42] O. O'Reilly, J. Nordström, J. E. Kozdon, and E. M. Dunham, Simulation of earthquake rupture dynamics in complex geometries using coupled finite difference and finite volume methods, Communications in Computational Physics, 17 (2015), pp. 337-370.

[43] C. Pelties, J. Puente, J.-P. Ampuero, G. B. Brietzke, and M. KÄser, Three-dimensional dynamic rupture simulation with a high-order discontinuous Galerkin method on unstructured tetrahedral meshes, Journal of Geophysical Research: Solid Earth, 117 (2012), pp. 1-15.

[44] P.-O. Persson and G. Strang, A simple mesh generator in MATLAB, SIAM review, 46 (2004), pp. 329-345.

[45] E. Pipping, O. Sander, And R. Kornhuber, Variational formulation of rate-and state-dependent friction problems, ZAMM-Journal of Applied Mathematics and Mechanics/Zeitschrift für Angewandte Mathematik und Mechanik, 95 (2015), pp. 377-395.

[46] A. N. Poliakov, R. Dmowska, and J. R. Rice, Dynamic shear rupture interactions with fault bends and off-axis secondary faulting, Journal of Geophysical Research: Solid Earth, 107 (2002).

[47] L. J. Pyrak-Nolte, L. R. Myer, And N. G. Cook, Transmission of seismic waves across single natural fractures, Journal of Geophysical Research: Solid Earth, 95 (1990), pp. 8617-8638.

[48] J. R. Rice, N. Lapusta, And K. Ranjith, Rate and state dependent friction and the stability of sliding between elastically deformable solids, Journal of the Mechanics and Physics of Solids, 49 (2001), pp. 1865-1898.

[49] B. Riviere And M. F. Wheeler, Discontinuous finite element methods for acoustic and elastic wave problems, Contemporary Mathematics, 329 (2003), pp. 271-282

[50] A. Rubin And J.-P. Ampuero, Earthquake nucleation on (aging) rate and state faults, Journal of Geophysical Research: Solid Earth, 110 (2005).

[51] M. Schoenberg, Elastic wave behavior across linear slip interfaces, The Journal of the Acoustical Society of America, 68 (1980), pp. 1516-1521.

[52] Z. Shi And Y. Ben-Zion, Dynamic rupture on a bimaterial interface governed by slip-weakening friction, Geophysical Journal International, 165 (2006), pp. 469-484.

[53] H. Shlomai and J. Fineberg, The structure of slip-pulses and supershear ruptures driving slip in bimaterial friction, Nature communications, 7 (2016), p. 11787.

[54] H. Si, Tetgen, a Delaunay-based quality tetrahedral mesh generator, ACM Transactions on Mathematical Software (TOMS), 41 (2015), p. 11.

[55] J. Tago, V. M. Cruz-Atienza, J. Virieux, V. Etienne, and F. J. SÁnchez-Sesma, A $3 D$ hp-adaptive discontinuous Galerkin method for modeling earthquake dynamics, Journal of Geophysical Research: Solid Earth, 117 (2012), pp. 1-21.

[56] E. Tessmer, D. Kosloff, And A. Behle, Elastic wave propagation simulation in the presence of surface topography, Geophysical Journal International, 108 (1992), pp. 621-632.

[57] J. Vermylen, M. D. Zoback, ET AL., Hydraulic fracturing, microseismic magnitudes, and stress evolution in the Barnett Shale, Texas, USA, in SPE Hydraulic Fracturing Technology Conference, Society of Petroleum Engineers, 2011.

[58] Y. Wang And S. M. Day, Seismic source spectral properties of crack-like and pulse-like modes of dynamic rupture, Journal of Geophysical Research: Solid Earth, (2017).

[59] Y. Wang, S. M. Day, and M. A. Denolle, Geometric controls on pulse-like rupture in a dynamic model of the 2015 Gorkha earthquake, Journal of Geophysical Research: Solid Earth, 124 (2019), pp. 1544-1568.

[60] T. Warburton and J. S. Hesthaven, On the constants in hp-finite element trace inverse inequalities, Computer methods in applied mechanics and engineering, 192 (2003), pp. 2765-2773.

[61] L. C. Wilcox, G. Stadler, C. Burstedde, and O. Ghattas, A high-order discontinuous Galerkin method for wave propagation through coupled elastic-acoustic media, Journal of Computational Physics, 229 (2010), pp. 9373-9396.

[62] S. Wollherr, A.-A. Gabriel, And C. UphOfF, Off-fault plasticity in three-dimensional dynamic rupture simulations using a modal discontinuous Galerkin method on unstructured meshes: implementation, verification and application, Geophysical Journal International, 214 (2018), pp. 1556-1584.

[63] S. Xu, Y. Ben-Zion, J.-P. Ampuero, And V. Lyakhovsky, Dynamic ruptures on a frictional interface with off-fault brittle damage: feedback mechanisms and effects on slip and near-fault motion, Pure and Applied Geophysics, 172 (2015), pp. 1243-1267.

[64] X. Xu, Z. Zhang, F. Hu, And X. Chen, Dynamic rupture simulations of the 1920 M s 8.5 Haiyuan Earthquake in China, Bulletin of the Seismological Society of America, (2019).

[65] R. Ye, M. V. De Hoop, C. L. Petrovitch, L. J. Pyrak-Nolte, and L. C. Wilcox, A discontinuous Galerkin method with a modified penalty flux for the propagation and scattering of acousto-elastic waves, Geophysical Journal International, (2016).

[66] R. Ye, K. Kumar, M. V. De Hoop, And M. Campillo, Analysis of dynamic ruptures generating seismic waves in a self-gravitating planet: an iterative coupling scheme and well-posedness, Quarterly of Applied Mathematics, (2019). In print.

[67] Q. Zhan, Q. Sun, Q. Ren, Y. Fang, H. Wang, And Q. H. LiU, A discontinuous Galerkin method for simulating the effects of arbitrary discrete fractures on elastic wave propagation, Geophysical Journal International, 210 (2017), pp. 1219-1230.

[68] Q. Zhan, M. Zhuang, Y. MaO, AND Q. H. LiU, Unified riemann solution for multi-physics coupling: 
Anisotropic poroelastic/elastic/fluid interfaces, Journal of Computational Physics, (2019), p. 108961.

[69] Z. Zhang, W. ZhANG, AND X. Chen, Three-dimensional curved grid finite-difference modelling for non-planar rupture dynamics, Geophysical Journal International, 199 (2014), pp. 860-879. 